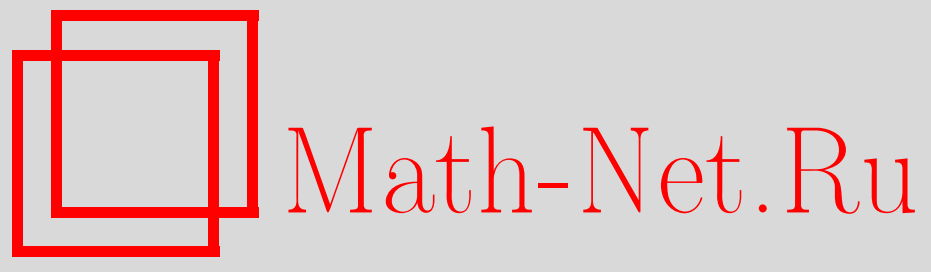

В. В. Федорчук, Слабо бесконечномерные пространства, УМH, 2007, том 62, выпуск 2, 109-164

DOI: https://doi.org/10.4213/rm6212

Использование Общероссийского математического портала Math-Net.Ru подразумевает, что вы прочитали и согласны с пользовательским соглашением http://www . mathnet.ru/rus/agreement

Параметры загрузки:

IP: 35.173 .137 .237

26 апреля 2023 г., 16:45:32

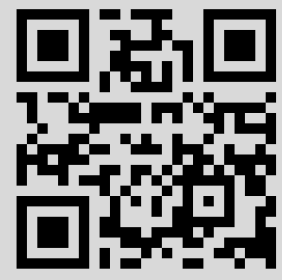




\section{Слабо бесконечномерные пространства}

\section{В. В. Федорчук}

Обзор посвящен двум сериям новых классов пространств, помежуточных между классом слабо бесконечномерных в смысле П. С. Александрова пространств и классом $C$-пространств, а именно классам $m$ - $C$-пространств и $w-m$ - $C$-пространств, $m=2,3, \ldots, \infty$. Классы $2-C$ и $w$-2- $C$ совпадают с классом слабо бесконечномерных пространств, а компактные $\infty$ - $C$-пространства - это в точности $C$-компакты Хэйвера. На эти классы распространяются основные результаты теории слабо бесконечномерных пространств, включая классификацию посредством трансфинитных лебеговых размерностей и индексов Лузина-Серпинского. Слабые $m-C$ пространства характеризуются посредством существенных отображений в $m$-компакты Хендерсона. Доказывается существование наследственно $m$-сильно бесконечномерных пространств.

Библиография: 102 названия.

\section{СоДЕРЖАНИЕ}

Введение

$\S 1$ Различные классы слабо бесконечномерных пространств. Теоремы сложения, суммы и монотонности ..................... 111

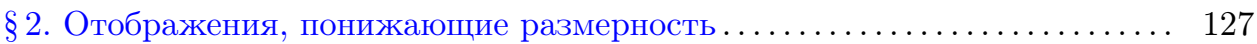

$\S 3$. Отображения, повышающие размерность.................. 132

$\S 4$. Трансфинитные продолжения лебеговой размерности и размерность

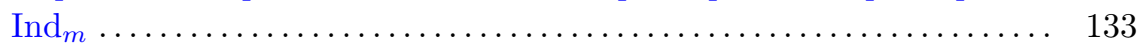

$\S 5$. Факторизационные теоремы и компактификации ................ 146

$\S 6$. Существенные отображения и $m$-компакты Хендерсона.............. 151

$\S 7$. Наследственно сильно бесконечномерные компакты............. 155

Добавление. Промежуточные размерности бикомпактов .............. 158

Список литературы .................................... 160

\section{Введение}

7 мая 2006 г. исполнилось 110 лет со дня рождения Павла Сергеевича Александрова - создателя советской топологической школы, получившей всемирное

Работа выполнена при поддержке гранта РФФИ 06-01-00764 и гранта РНП 2.1.1. 7988.

(C) В. В. ФЕдорчук, 2007 
признание. П. С. Александров, будучи учеником Н. Н. Лузина, начал свою научную работу в области дескриптивной теории множеств. В 1915 г. он доказал, что всякое несчетное борелевское множество (на прямой) имеет мощность континуума.

В начале 20-х годов П. С. Александров вместе с П. С. Урысоном основал и развил теорию бикомпактных пространств. Затем он посвятил себя геометрическому направлению в топологии. На основе введенного им понятия нерва покрытия были доказаны теоремы об аппроксимации компактов и более общих пространств полиэдрами. Вершинами творчества П. С. Александрова стали созданная им в конце 20 -х-начале 30 -х годов гомологическая теория размерности и написанная зимой 1941-42 г. знаменитая "Казанская" работа [1] о гомологических свойствах расположения комплексов и замкнутых множеств. "Казанская" работа завершила большой цикл исследований, начавшийся с построения гомологической теории компактов. За этот цикл исследований П. С. Александрову в 1943 г. была присуждена Сталинская премия 1-й степени.

Основная теорема гомологической теории размерности в современной форме гласит: для всякого конечномерного компакта $X$ имеет место равенство $\operatorname{dim} X=\operatorname{dim}_{\mathbb{Z}} X$, где $\operatorname{dim}_{\mathbb{Z}} X$ - когомологическая размерность компакта $X$ с коэффициентами в группе $\mathbb{Z}$, равная наибольшему числу $n$ такому, что найдется замкнутое множество $A \subset X$, для которого $H^{n}(X, A ; \mathbb{Z}) \neq 0$. П. С. Александров получил этот результат, опираясь на предложенную им характеристику размерности $\operatorname{dim}$ в терминах продолжения отображений в сферы и на теорему Хопфа о продолжении отображений, и поставил вопрос о равенстве $\operatorname{dim}=\operatorname{dim}_{\mathbb{Z}}$ в классе всех метризуемых компактов. Эта проблема П. С. Александрова оставалась нерешенной более полувека, пока в 1987 г. А. Н. Дранишников [2] не построил бесконечномерный компакт $X$ когомологической размерности $\operatorname{dim}_{\mathbb{Z}} X=3$.

В 1948 г. в предисловии к русскому переводу монографии В. Гуревича и Г. Волмэна [3] П. С. Александров рассмотрел понятие слабой бесконечномерности. Исследования в этом направлении, начавшиеся 10 лет спустя в работах Б. Т. Левшенко [4] и Е.Г. Скляренко [5], быстро показали, какое важное место занимают слабо бесконечномерные пространства (или кратко widпространства) в классе всех бесконечномерных пространств. Была создана стройная внутренняя теория wid-пространств, построены примеры, демонстрирующие взаимоотношения класса wid-пространств с другими классами бесконечномерных пространств. Отметим здесь: 1) теорему Хендерсона [6] о том, что всякий сильно бесконечномерный компакт содержит континуум, любой подконтинуум которого бесконечномерен; 2) пример Р. Поля [7] несчетномерного wid-компакта; 3) совсем недавний пример Борста [8] wid-компакта, не являющегося $C$-пространством.

Свойство $C$ для метрических пространств в 1973 г. ввел В. Хэйвер [9], доказавший, что локально стягиваемое метрическое пространство, представимое в виде объединения счетного числа компактов со свойством $C$, является ANRпространством. Топологическое определение $C$-пространств было предложено в 1978 г. Д. Аддисом и Дж. Грешэмом [10]. Выяснилось, что $C$-пространства играют важную роль в теории клеточно-подобных отображений [11], в теории селекций многозначных отображений [12]-[14] и других разделах топологии. 
В [15] начато изучение $m$ - $C$-пространств, промежуточных между wid- и $C$-пространствами:

$$
\text { wid }=2-C \supset 3-C \supset \cdots \supset m-C \supset \cdots \supset \infty-C \supset C .
$$

В настоящей работе вводится понятие слабого $m$ - $C$-пространства, или $w$ - $m$ - $C$ пространства, с естественным соотношением $m-C \subset w-m-C$ и аналогичной $(0.1)$ последовательностью

$$
\operatorname{wid}=w-2-C \supset \cdots \supset w-m-C \supset \cdots \supset w-\infty-C \supset w C \supset C .
$$

На $m$ - $C$ - и $w-m-C$-пространства переносятся практически все результаты, касающиеся wid-пространств. Более того, автору пока не известны примеры пространств, которые противоречили бы одному из равенств

$$
m-C=m+1-C, \quad w-m-C=w-m+1-C, \quad m-C=w-m-C .
$$

Возникает большое число и других вопросов, с которыми можно познакомиться в [16].

Статья не является всеобъемлющим обзором теории слабо бесконечномерных пространств. Дополнительную информацию по рассматриваемой теме читатель может найти в [15], [17]-[19] и в статьях, приведенных в списке литературы. Многие результаты приведены без доказательств. В первую очередь это относится к $\S 6$ и добавлению, которые в развернутом виде будут опубликованы отдельно.

\section{§ 1. Различные классы слабо бесконечномерных пространств. Теоремы сложения, суммы и монотонности}

Бесконечномерность как отрицание конечномерности начинает становиться содержательным понятием, если мы обращаемся к различным, но эквивалентным (например, для метрических компактов) определениям конечной размерности. Создатель теории размерности П. С. Урысон доказал, что для произвольного метризуемого компакта $X$ имеет место равенство

$$
\operatorname{dim} X=\operatorname{ind} X=\operatorname{Ind} X,
$$

впоследствии названное тождеством Урысона. Основным размерностным инвариантом в исследованиях П.С. Урысона была введенная им малая индуктивная размерность, которую он просто называл размерностью. Летом 1922 г. П. С. Урысон писал (опубликовано в [20]):

"Вместо бесконечной размерности можно было бы ввести трансфинитные размерности для всех чисел второго класса и, может быть, даже дальше, потому что размерность (согласно ее определению) есть порядковое число. Впрочем, это распространение, по крайней мере в данный момент, кажется мне лишенным интереса, тем более, что даже в этом случае останутся, по-видимому, множества, не укладывающиеся в классификацию (области гильбертова пространства)". 
Формальное определение малой трансфинитной размерности ind было дано В. Гуревичем [21] в 1928 г., а большая трансфинитная размерность Ind была определена Ю. М. Смирновым [22] лишь в 1959 г. К настоящему времени теория трансфинитных размерностей сложилась в хорошо разработанную область бесконечномерной топологии, с которой читатель может познакомиться в монографиях [17], [18] и обзорах [23], [24].

П. С. Урысон охарактеризовал $n$-мерные пространства как объединения нульмерных подпространств в количестве $n+1$ штук. В последний год своей жизни он владел понятием счетномерного пространства, т.е. пространства, являющегося суммой счетного числа конечномерных, а значит, и нульмерных (в классе сепарабельных метрических пространств) множеств. П. С. Урысон ставил вопрос о связи этого понятия с трансфинитной размерностью и высказал гипотезу [25] о несчетномерности гильбертова куба. Формальное определение счетномерного пространства (в случае метрических пространств) было дано В. Гуревичем в [21], где он доказал несчетномерность гильбертова куба. В монографии В. Гуревича и Г. Волмэна [26] показано, что сепарабельное полное метрическое пространство счетномерно тогда и только тогда, когда для него определена малая трансфинитная размерность. Таким образом, исследование счетномерных пространств во многом сводится к изучению пространств, для которых определена трансфинитная размерность.

Третий тип бесконечномерности на основе понятия перегородки был введен П. С. Александровым [3] в 1948 г.

ОПРЕДЕЛЕНИЕ 1.1. Нормальное пространство $X$ называется слабо бесконечномерным, если для любой последовательности $\left(F_{1}^{i}, F_{2}^{i}\right), i \in \omega$, дизъюнктных пар замкнутых в $X$ множеств существуют перегородки $P_{i}$ между $F_{1}^{i}$ и $F_{2}^{i}$ с пустым пересечением. Пространство, не являющееся слабо бесконечномерным, называется сильно бесконечномерным.

Несколько лет спустя Ю. М. Смирнов предложил другое определение слабой бесконечномерности. В нем требуется, чтобы пересечение конечного числа перегородок $P_{i}$ было пусто. Такие пространства будем называть $S$-слабо бесконечномерными. В классе бикомпактов (и даже счетно компактных пространств) понятия слабой и $S$-слабой бесконечномерности совпадают. Дискретная сумма конечномерных кубов $\bigoplus I^{n}$ показывает, что $S$-слабо бесконечномерные пространства образуют собственный подкласс класса слабо бесконечномерных пространств.

В 1973 г. В. Хэйвер [9] для метрического пространства $X$ определил свойство $C$, которое состоит в следующем:

для всякой последовательности $\varepsilon_{i}, i \in \omega$, положительных чисел существует такая последовательность $\left\{v_{i}\right\}$ дизъюнктных открытых в $X$ семейств, что $\operatorname{diam} V<\varepsilon_{i}$ для каждого $V \in v_{i}$ и $X=\bigcup\left\{V: V \in v_{i}, i \in \omega\right\}$.

Хэйвер заметил, что всякое счетномерное метрическое пространство обладает свойством $C$, и доказал [27], что всякое локально стягиваемое метрическое пространство, представимое в виде счетного объединения компактов со свойством $C$, является ANR-пространством. В 1978 г. Аддис и Грешэм [10] 
предложили топологическую версию свойства $C$, не накладывая на пространство никаких ограничений. Но мы будем рассматривать только нормальные пространства (включая аксиому $T_{1}$ ).

ОПРЕДЕЛЕНИЕ 1.2. Нормальное пространство $X$ называется $C$-пространством $(X \in C)$, если для любой последовательности $\left(u_{i}\right), i \in \omega$, его открытых покрытий существует последовательность $\left(v_{i}\right)$ дизъюнктных открытых семейств пространства $X, C$-вписанная в последовательность $\left(u_{i}\right)$. Последнее означает, что каждое семейство $v_{i}$ вписано в покрытие $u_{i}$, а совокупность семейств $v_{i}$ является покрытием пространства $X$, т.е.

$$
X=\bigcup\left\{\bigcup v_{i}: i \in \omega\right\}
$$

В этом случае говорят также, что последовательность $\left(u_{i}\right)$ (бесконечная или конечная) несущественна.

Непосредственно из определения вытекает, что всякое $C$-пространство $X$ является просеянным в смысле Бинга [28], т.е. в любое открытое покрытие пространства $X$ можно вписать открытое покрытие, распадающееся в сумму счетного числа дизъюнктных семейств. Поэтому из теоремы Нагами [29] о паракомпактности всякого нормального счетно паракомпактного просеянного пространства вытекает следующее предложение.

ПРЕДЛОЖЕНИЕ 1.3. Всякое счетно паракомпактное $C$-пространство паракомпактно.

Это утверждение дает нам большой запас конечномерных пространств, не являющихся $C$-пространствами. Таковым будет, например, нульмерное пространство $\omega_{1}$ всех счетных трансфинитов.

ЗАмечАниЕ 1.4. В классе метрических компактов определение Хэйвера совпадает с определением Аддиса и Грешэма. Из нижеследующего предложения 1.5 вытекает, что всякое метрическое пространство, представимое в виде объединения счетного числа компактов со свойством $C$, является $C$-пространством. Но в то же время не всякое польское $C$-пространство обладает свойством $C$. В самом деле, упомянутый во введении несчетномерный wid-компакт Р. Поля является $C$-пространством, содержащим сильно бесконечномерное подпространство $Y$. Следовательно, $Y$ не является $C$-пространством согласно предложению 1.6. В то же время $Y$ имеет свойство $C$, поскольку оно наследуется при переходе к подпространству.

ПреДЛОЖЕНИЕ 1.5 [10]. Если наследственно коллективно нормальное пространство $X$ может быть представлено в виде объединения счетного числа подпространств $X_{i} \in C$, то $X \in C$.

ПрЕДЛОЖЕНИЕ 1.6 [10]. Всякое C-пространство слабо бесконечномерно.

С применением предложенной $\Phi$. Острандом [30] характеристики размерности $\operatorname{dim}$ доказывается

ПРЕДЛОЖЕНИЕ 1.7 [10]. Всякое паракомпактное конечномерное пространство является C-пространством. 
Из предложений 1.5 и 1.7 вытекает следующая теорема.

ТеОрема 1.8 [10]. Всякое наследственно паракомпактное счетномерное пространство является C-пространством.

Таким образом, ввиду упомянутых выше примеров Р. Поля и П. Борста метрические $C$-пространства занимают строго промежуточное положение между метрическими счетномерными и метрическими wid-пространствами. Не известен ответ на следующий вопрос.

Вопрос 1. Является ли $C$-пространством всякое паракомпактное счетномерное пространство?

В то же время, за пределами паракомпактов эти классы различаются. Как отмечено выше, не всякое нульмерное пространство является $C$-пространством. Предложение 1.3 показывает, что определение $C$-пространства выделяет не только размерностные свойства пространства, но и свойства типа компактности. В.А. Чатырко предложил [31] в определении $C$-пространства ограничиться локально конечными покрытиями. При этом получается более широкий класс пространств, содержащий все конечномерные пространства. Ниже мы укажем некоторые отличия теории $C$-пространств от теории нового класca lf- $C$-пространств. Основным недостатком этого нового класса пространств является то, что нельзя автоматически доказать принадлежность классу lf- $C$ замкнутых подмножеств lf- $C$-пространств (см. предложение 1.23 ).

Но прежде чем перейти к новым классам пространств и к параллельному изложению новых возникающих при этом теорий, вернемся к предложению 1.6. Почему всякое $C$-пространство $X$ слабо бесконечномерно? Для последовательности $\left(F_{1}^{i}, F_{2}^{i}\right), i \in \omega$, дизъюнктных подмножеств пространства $X$ надо найти перегородки $P_{i}$ между $F_{1}^{i}$ и $F_{2}^{i}$ с пустым пересечением $\bigcap P_{i}$. Воспользовавшись нормальностью пространства $X$, найдем такие окрестности $O F_{1}^{i}$ и $O F_{2}^{i}$, что $\left[O F_{1}^{i}\right] \cap\left[O F_{2}^{i}\right]=\varnothing$. Полагая $U_{1}^{i}=X \backslash\left[O F_{2}^{i}\right], U_{2}^{i}=X \backslash\left[O F_{1}^{i}\right]$, получаем бинарные открытые покрытия $u_{i}=\left\{U_{1}^{i}, U_{2}^{i}\right\}$ пространства $X$. Поскольку $X \in C$, существует последовательность $\left(v_{i}\right), i \in \omega$, дизъюнктных открытых семейств, $C$-вписанная в последовательность $\left(u_{i}\right)$. Полагаем теперь

$$
O_{1}^{i}=O F_{1}^{i} \cup \operatorname{St}\left(O F_{1}^{i}, v_{i}\right), \quad O_{2}^{i}=O F_{2}^{i} \cup\left(\bigcup\left\{V \in v_{i}: V \cap O F_{1}^{i}=\varnothing\right\}\right) .
$$

Так как семейство $v_{i}$ вписано в покрытие $u_{i}$, ни один из элементов $V \in v_{i}$ не пересекается одновременно с двумя множествами $O F_{1}^{i}$ и $O F_{2}^{i}$. Поэтому множества $O_{1}^{i}$ и $O_{2}^{i}$ являются непересекающимися окрестностями множеств $F_{1}^{i}$ и $F_{2}^{i}$, а множества $P_{i}=X \backslash O_{1}^{i} \cup O_{2}^{i}$ - перегородками между $F_{1}^{i}$ и $F_{2}^{i}$. Наконец, из $(1.2)$ вытекает, что $\bigcup\left(\bigcup v_{i}\right) \subset O_{1}^{i} \cup O_{2}^{i}$. Поэтому $\bigcap_{i} P_{i}=\varnothing$ в силу (1.1).

Итак, при доказательстве предложения 1.6 у нас возникли бинарные покрытия $u_{i}=\left\{U_{1}^{i}, U_{2}^{i}\right\}$, рассмотрение которых и привело к слабой бесконечномерности пространства. Это приводит к простой идее определения целого спектра классов пространств, промежуточных между $C$-пространствами и widпространствами: в определении $C$-пространства надо ограничить класс рассматриваемых покрытий. 
ОПРЕДЕЛЕНИЕ 1.9. Пусть $\mathscr{P}$ - класс открытых покрытий топологических пространств. Нормальное пространство $X$ называется $\mathscr{P}$ - $C$-пространством (кратко $X \in \mathscr{P}-C$ ), если всякая последовательность $\left(u_{i}\right), i \in \omega$, его покрытий из класса $\mathscr{P}$ несущественна.

Возникают следующие классы пространств:

1) $k$-C-nространства, $k$ - натуральное число $\geqslant 2, \mathscr{P}$ состоит из покрытий, содержащих не более чем $k$ элементов (всякое нормальное пространство является 1-C-пространством);

2) $\infty$-C-пространства, $\mathscr{P}$ состоит из всех конечных покрытий;

3) $\omega_{0}$-C-пространства, $\mathscr{P}$ состоит из всех счетных покрытий;

4) sf-C-пространства, $\mathscr{P}$ состоит из всех звездно конечных покрытий;

5) lf-C-пространства, $\mathscr{P}$ состоит из всех локально конечных покрытий;

6) pf-C-пространства, $\mathscr{P}$ состоит из всех точечно конечных покрытий.

Кроме 1), 2), 3), можно рассматривать и другие ограничения на мощность покрытий, комбинируя их с ограничениями типа 4), 5), 6).

Имеет место очевидное предложение.

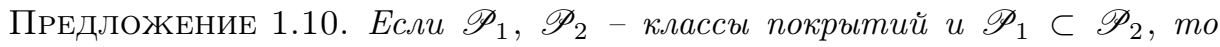
$\mathscr{P}_{2}-C \subset \mathscr{P}_{1}-C$.

Таким образом, возникает следующая цепочка классов пространств:

$$
C \subset \omega_{0}-C \subset \infty-C \subset \cdots \subset k-C \subset \cdots \subset 2-C
$$

Наибольший член этой последовательности, как мы видели, совпадает с классом wid-пространств. Если мы не выходим за пределы бикомпактных пространств, то классы $C, \omega_{0}-C$ и $\infty$ - $C$ совпадают. Поскольку основные проблемы, касающиеся слабо бесконечномерных пространств, не решены для метризуемых компактов, нас в первую очередь будут интересовать классы конечных покрытий. В связи с этим введем в рассмотрение упорядоченное по типу $\omega+1$ множество $\overline{\mathbb{N}}$, состоящее из натуральных чисел $k \geqslant 2$ и элемента $\infty$, превосходящего все $k$. Множество $\overline{\mathbb{N}}$ удобно тем, что многие утверждения об $m$ - $C$ пространствах, $m \in \overline{\mathbb{N}}$, доказываются единым способом, не зависящим от $m$. В дальнейшем буквой $m$ будем обозначать только элементы множества $\overline{\mathbb{N}}$.

Определение $\mathscr{P}$ - $C$-пространства является обобщением определения $C$-пространства. Обратимся теперь к определению wid-пространства. Нахождение перегородок $P_{i}$ между непересекающимися замкнутыми множествами $F_{1}^{i}$ и $F_{2}^{i}$, подчиненных условию $\bigcap_{i} P_{i}=\varnothing$, равносильно нахождению дизъюнктных пар окрестностей $O F_{1}^{i}$ и $O F_{2}^{i}$, в совокупности покрывающих все пространство. Но вместо дизъюнктных пар $\left(F_{1}^{i}, F_{2}^{i}\right)$ можно рассматривать дизъюнктные семейства других классов: дизъюнктные тройки, дизъюнктные конечные семейства и т.д. Это приводит к определению классов пространств, более узких, чем класс wid-пространств. Предварительно приведем несколько вспомогательных понятий.

ОПРЕДЕЛЕНИЕ 1.11. Последовательность $k_{i}, i \in \omega$, натуральных чисел $\geqslant 2$ будем называть $m$-последователъностъю, если все $k_{i}=m$ при конечном $m$. 
Семейство открытых (замкнутых) подмножеств пространства $X$, состоящее не более чем из $k$ элементов, будем называть открытым (замкнутым) $k$ семейством пространства $X$. Дизъюнктные замкнутые $k$-семейства будем называть $k$-системами. Открытые $k$-семейства, являющиеся покрытиями, будем называть $k$-покрытиями пространства $X$.

Пусть $\Phi=\left\{F_{1}, \ldots, F_{k}\right\}-k$-система пространства $X$ (в записи $k$-семейства мы предполагаем, что оно состоит ровно из $k$ множеств, некоторые из которых могут быть пустыми). Всякое дизъюнктное семейство $O \Phi=\left\{O F_{1}, \ldots, O F_{k}\right\}$ окрестностей элементов $\Phi$ называется окрестностъю системы $\Phi$. Если $k$-система $\Phi$ комбинаторно вписана в $k$-покрытие $u$, то покрытие $u$ будем называть $k$-накрытием системы $\Phi$, а пару $(u, \Phi)-k$-накрывающей парой.

ОПРЕДЕЛЕНИЕ 1.12. Нормальное пространство $X$ называется $w-m-C$-npoстранством $(X \in w-m-C)$, если для любой $m$-последовательности $\left(k_{i}\right)$ всякая последовательность $\left(\Phi_{i}\right) \quad k_{i}$-систем пространства $X$ несущественна, т.е. существуют такие окрестности $O \Phi_{i}$, что семейство $\bigcup\left\{O \Phi_{i}: i \in \omega\right\}$ является покрытием пространства $X$. Очевидно, в этом определении можно ограничиться рассмотрением лишь функционально замкнутых систем.

ПРЕДЛОЖЕНИЕ 1.13. Всякое $m$-C-nространство является $w$-m-C-nространством.

ДокАЗАТЕЛЬство. Фиксируем $m$-последовательность $\left(k_{i}\right)$ и последовательность $\left(\Phi_{i}=\left\{F_{1}^{i}, \ldots, F_{k_{i}}^{i}\right\}\right) \quad k_{i}$-систем $m$ - $C$-пространства $X$. Поскольку пространство $X$ нормально, существуют такие окрестности $O_{1} F_{j}^{i}$ множеств $F_{j}^{i}$, что

$$
\left[O_{1} F_{j_{1}}^{i}\right] \cap\left[O_{1} F_{j_{2}}^{i}\right]=\varnothing, \quad \text { если } j_{1} \neq j_{2} .
$$

Полагаем $u_{i}=\left\{U_{1}^{i}, \ldots, U_{k_{i}}^{i}\right\}$, где

$$
U_{j}^{i}=X \backslash \bigcup\left\{\left[O_{1} F_{l}^{i}\right]: l \neq j\right\} .
$$

Из (1.3) и (1.4) вытекает, что $u_{i}$ является открытым покрытием пространства $X$. По определению $m$ - $C$-пространства существует последовательность $\left(v_{i}\right)$ дизъюнктных открытых в $X$ семейств, $C$-вписанная в последовательность $\left(u_{i}\right)$. При этом, переходя к укрупнениям семейств $v_{i}$, можно считать, что каждое $v_{i}$ комбинаторно вписано в $u_{i}$, т.е. $v_{i}=\left\{V_{1}^{i}, \ldots, V_{k_{i}}^{i}\right\}$ и $V_{j}^{i} \subset U_{j}^{i}$. Полагаем $O F_{j}^{i}=O_{1} F_{j}^{i} \cup V_{j}^{i}$. Покажем, что $O \Phi_{i}=\left\{O F_{1}^{i}, \ldots, O F_{j}^{i}\right\}$ является окрестностью системы $\Phi_{i}$. Для этого надо проверить, что

$$
O F_{j_{1}}^{i} \cap O F_{j_{2}}^{i}=\varnothing, \quad \text { если } j_{1} \neq j_{2}
$$

Из (1.4) и включений $V_{j}^{i} \subset U_{j}^{i}$ вытекают равенства $O_{1} F_{j_{1}}^{i} \cap V_{j_{2}}^{i}=\varnothing=O_{1} F_{j_{2}}^{i} \cap V_{j_{1}}^{i}$, которые вместе с (1.3) и дизъюнктностью семейств $v_{i}$ гарантируют выполнение равенства (1.5). Семейство $\bigcup\left\{O \Phi_{i}: i \in \omega\right\}$ является покрытием пространства $X$ в силу включений $V_{j}^{i} \subset O F_{j}^{i}$. Предложение 1.13 доказано.

Следующее утверждение очевидно.

ПреДЛОЖЕНИЕ 1.14. Если $m_{1}<m_{2}$, mo $w-m_{2}-C \subset w-m_{1}-C$. 
В определении 1.12 можно было бы рассмотреть и бесконечные системы множеств. Но в этом случае вместо дизъюнктности надо предполагать дискретность этих систем.

ОПРЕДЕЛЕНИЕ 1.15. Коллективно нормальное пространство $X$ называется $w$ - $C$-пространством $(X \in w-C)$, если любая последовательность $\left(\Phi_{i}\right)$ дискретных семейств замкнутых множеств пространства $X$ несущественна.

Так же, как предложение 1.13, доказывается следующее утверждение.

ПРЕДЛОЖЕНИЕ 1.16. Всякое коллективно нормальное $C$-пространство является $w$-C-пространством.

Предложения 1.13 и 1.16 позволяют называть $w$-m- $C$-пространства слабыми m-C-пространствами, а $w$-C-пространства слабыми $C$-пространствами.

Вопрос 2. Всякое ли слабое $m$ - $C$-пространство является $m$ - $C$-пространством?

Положительный ответ очевиден при $m=2$. Что касается $m \geqslant 3$, ответ на вопрос 2 не известен и в классе метризуемых компактов.

Вопрос 3. Всякое ли паракомпактное слабое $C$-пространство является $C$-пространством?

Следующее утверждение дает нам широкий спектр слабых $C$-пространств, не являющихся $C$-пространствами (все конечномерные счетно компактные, но не бикомпактные пространства).

ПРЕДЛОЖЕНИЕ 1.17. Всякое конечномерное счетно компактное пространство является слабым C-пространством.

Поскольку всякое дискретное семейство подмножеств счетно компактного пространства конечно, предложение 1.17 вытекает из следующей упомянутой выше теоремы Остранда.

Tеорема 1.18 [30]. Если $\operatorname{dim} X \leqslant n$, то во всякое локально конечное открытое покрытие пространства $X$ можно вписать открытое покрытие, представимое в виде обгединения не более чем $n+1$ дискретных в $X$ семейств множеств.

Гипотетически имеется еще одна возможность получения новых классов слабо бесконечномерных пространств. А именно, можно комбинировать определения 1.9 и 1.12 , рассматривая $k_{i}$-накрывающие пары $\left(u_{i}, \Phi_{i}\right)$ и пробуя найти последовательность $\left(O \Phi_{i}\right), C$-вписанную в последовательность $u_{i}$. Но, как показывает предложение 1.19 , при таком подходе мы не получаем ничего нового.

Конечное открытое покрытие $u$ пространства $X$ будем называть его $m$ покрытием, если $u$ состоит из $m$ элементов при $m \in \mathbb{N}$. Когда мы будем говорить о последовательности $m$-покрытий $u_{i}$ пространства $X$, всегда будет предполагаться, что фиксирована последовательность $k_{i}$ натуральных чисел и $\left|u_{i}\right|=k_{i}$. Последовательность $\left(v_{i}\right)$ называется комбинаторно $C$-вписанной в последовательность $\left(u_{i}\right)$, если она $C$-вписана и каждое семейство $v_{i}$ комбинаторно вписано в покрытие $u_{i}$. 
ПРЕДЛОЖЕНИЕ 1.19 [15]. Для нормального пространства Х следующие условия равносилъны.

1) $X$ является $m$-C-пространством.

2) Для любой последовательности $\left(u_{i}\right)$ m-покрытий пространства $X$ существует последовательность $\left(v_{i}\right)$ дизгюнктных открытых семейств, комбинаторно C-вписанная в последовательность $\left(u_{i}\right)$.

3) Для любой $m$-последовательности $\left(k_{i}\right)$ и любой последовательности $\left(u_{i}, \Phi_{i}\right) \quad k_{i}$-накрывающих пар существует последовательность $\left(O \Phi_{i}\right)$ окрестностей систем $\Phi_{i}, C$-вписанная в последовательность $\left(u_{i}\right)$.

ЗАмечАниЕ 1.20 . Если $m$ - $C$-пространство $X$ счетно паракомпактно, то в любую последовательность $\left(u_{i}\right)$ его $m$-покрытий можно комбинаторно $C$-вписать последовательность $\left(v_{i}\right)$ дизъюнктных функиионально открытых семейств.

ЗАмЕчаниЕ 1.21. По аналогии с $S$-слабо бесконечномерными пространствами вводятся понятия $S-m-C-, S-C-, S-w-m-C$ - и $S-w-C$-nространства требованием, чтобы уже некоторое конечное число семейств $v_{i}$ (окрестностей $O \Phi_{i}$ ) покрывало пространство $X$. При этом здесь уже не надо ограничиваться счетно паракомпактными пространствами, для того чтобы предполагать эти семейства функционально открытыми.

ЗАмЕчАниЕ 1.22. Почти все вновь введенные классы пространств замкнуты относительно перехода к замкнутым подпространствам. Но мы не можем этого утверждать про sf- $C$-, lf- $C$-, pf- $C$-пространства и $S$-модификации этих классов из замечания 1.21. Основной проблемой здесь является невозможность продолжения, например, открытого звездно-конечного покрытия замкнутого подпространства до открытого звездно-конечного покрытия всего пространства. Этого нельзя сделать даже в классе метрических пространств. В случае локально конечных и точечно конечных покрытий ситуация существенно лучше (см. предложения 1.23 и 1.24).

Вопрос 4. Замкнуты ли классы sf- $C$-, lf- $C$ - и pf- $C$-пространств относительно перехода к замкнутым подпространствам?

Частичный ответ на этот вопрос дают следующие утверждения.

ПРЕДЛОЖЕНИЕ 1.23. Всякое замкнутое подмножество счетно паракомпактного коллективно нормального lf-C-пространства является lf-C-nространством.

Это утверждение следует из теоремы Катетова [32] о том, что всякое локально конечное открытое покрытие замкнутого подмножества счетно паракомпактного коллективно нормального пространства $X$ можно продолжить до локально конечного открытого покрытия всего пространства $X$. Вытекает оно и из более ранней теоремы Даукера [33] о раздутии локально конечного семейства замкнутых подмножеств пространства $X$ до локально конечного открытого семейства.

ПРЕДЛОЖЕНИЕ 1.24. Всякое счетно паракомпактное коллективно нормальное lf-C-пространство является pf-C-пространством. 
Для доказательства достаточно применить теорему Э. Майкла [34] и К. Нагами [29] о том, что в точечно конечное открытое покрытие коллективно нормального пространства можно вписать $\sigma$-локально конечное открытое покрытие.

ПРЕДЛОЖЕНИЕ 1.25. Если $w$-m-C-пространство $X$ является счетно паракомпактно, то в определениии 1.12 можно считать, что окрестности $O \Phi_{i}=\left\{O F_{1}^{i}, \ldots, O F_{k_{i}}^{i}\right\}$ состоят из функционально открытых множеств.

При доказательстве используется то, что всякое счетное открытое покрытие счетно паракомпактного пространства можно ужать до замкнутого покрытия.

Лемма 1.26. Пусть (замкнутое) подмножество Y наследственно нормального (счетно паракомпактного) пространства $X$ является $w-m-C$-пространством. Тогда для любой т-последовательности $\left(k_{i}\right)$ и любой последовательности $\left(\Phi_{i}\right) \quad k_{i}$-систем пространства $X$ существуют такие их окрестности $O \Phi_{i}$, что $Y \subset \bigcup\left(\bigcup\left\{O \Phi_{i}: i \in \omega\right\}\right)$.

ДокАЗАтЕльство. Рассмотрим сначала случай счетно паракомпактного $X$ и замкнутого $Y$. Пусть $\Phi_{i}=\left\{F_{1}^{i}, \ldots, F_{k_{i}}^{i}\right\}$. Положим ${ }^{0} F_{j}^{i}=F_{j}^{i} \cap Y$ и $\Phi_{i}^{0}=$ $\left\{{ }^{0} F_{1}^{i}, \ldots,{ }^{0} F_{k_{i}}^{i}\right\}$. Согласно определению 1.12 , в $w$ - $m$ - $C$-пространстве $Y$ существуют такие окрестности $O \Phi_{i}^{0}=\left\{V_{1}^{i}, \ldots, V_{k_{i}}^{i}\right\}$ систем $\Phi_{i}^{0}$, что

$$
Y=\bigcup\left\{V_{j}^{i}: 1 \leqslant j \leqslant k_{i}, i \in \omega\right\}
$$

Положим $V_{i}=\bigcup\left\{V_{j}^{i}: 1 \leqslant j \leqslant k_{i}\right\}$. Поскольку счетная паракомпактность наследуется замкнутыми подмножествами, в открытое покрытие $v=\left\{V_{i}: i \in \omega\right\}$ пространства $Y$ можно комбинаторно вписать замкнутое покрытие $\gamma=\left\{\Gamma_{i}\right.$ : $i \in \omega\}$. Положим $\Gamma_{j}^{i}=\Gamma_{i} \cap V_{j}^{i}$. Поскольку $\Gamma_{i} \subset V_{i}$ и семейства $O \Phi_{i}^{0}$ дизъюнктны, множества $\Gamma_{j}^{i}$ замкнуты и

$$
Y=\bigcup\left\{\Gamma_{j}^{i}: 1 \leqslant j \leqslant k_{i}, i \in \omega\right\}
$$

Положим $H_{j}^{i}=\Gamma_{j}^{i} \cup F_{j}^{i}$. Семейства $h_{i}=\left\{H_{1}^{i}, \ldots, H_{k_{i}}^{i}\right\}$ дизъюнктны. Поэтому их можно заключить в окрестности $O h_{i}=\left\{O H_{1}^{i}, \ldots, O H_{k_{i}}^{i}\right\}$. Согласно (1.7), они и будут искомыми окрестностями $O \Phi_{i}$.

Для наследственно нормального $X$ и произвольного $Y$ сначала для каждого семейства $\Phi_{i}=\left\{F_{1}^{i}, \ldots, F_{k_{i}}^{i}\right\}$ найдем окрестности $O_{1} \Phi_{i}=\left\{G_{1}^{i}, \ldots, G_{k_{i}}^{i}\right\}$ с условием $\left[G_{j_{1}}^{i}\right] \cap\left[G_{j_{2}}^{i}\right]$ при $j_{1} \neq j_{2}$. Затем полагаем ${ }^{0} F_{j}^{i}=\left[G_{j}^{i}\right] \cap Y$ и $\Phi_{i}^{0}=\left\{{ }^{0} F_{1}^{i}, \ldots,{ }^{0} F_{k_{i}}^{i}\right\}$. В $Y$ находим окрестности $O \Phi_{i}^{0}=\left\{V_{1}^{i}, \ldots, V_{k_{i}}^{i}\right\}$, удовлетворяющие условию (1.6). Дизъюнктные семейства $\left\{V_{1}^{i}, \ldots, V_{k_{i}}^{i}\right\}$ можно продолжить до дизъюнктных открытых в $X$ семейств $\left\{U_{1}^{i}, \ldots, U_{k_{i}}^{i}\right\}$ согласно нижеследующей лемме 1.27. Полагая $O F_{j}^{i}=U_{j}^{i} \cup G_{j}^{i} \backslash \bigcup\left\{\left[G_{j^{\prime}}^{i}: j^{\prime} \neq j\right\}\right.$, получаем искомые окрестности $O \Phi_{i}=\left\{O F_{1}^{i}, \ldots, O F_{k_{i}}^{i}\right\}$. Лемма 1.26 доказана.

Следующее утверждение, уже частично использованное нами в предыдущем доказательстве, общеизвестно. Мы приводим его только для удобства дальнейших ссылок. 
ЛЕммА 1.27 (о продолжении дизъюнктных открытых семейств). Пусть $Y$ подпространство пространства $X u\left\{U_{1}, \ldots, U_{s}\right\}$ - конечное дизбюнктное семейство открытых подмножеств пространства $Y$. Тогда дизбюнктное семейство открытых в $X$ множеств $\left\{V_{1}, \ldots, V_{s}\right\}$ со свойством $V_{i} \cap Y=U_{i}$ существует в следующих случаях:

1) $X$ наследственно нормально;

2) $X$ счетно паракомпактно, $Y$ замкнуто, а $U_{i}$ функционально открьты в $Y$ (в этом случае $V_{i}$ можно выбрать функционально открытыми в $X)$.

ТЕОРема 1.28 (теорема сложения). Если наследственно нормалъное пространство $X$ можно представить в виде обгединения счетного семейства $w$-m-C-пространств $Y_{j}$, то $X$ является $w$-m-C-пространством.

При доказательстве используется стандартный прием. Последовательность $\varphi=\left(\Phi_{i}\right) \quad k_{i}$-систем пространства $X$ разбивается в дизъюнктную сумму бесконечных последовательностей $\varphi_{j}$. Затем к каждой последовательности $\varphi_{j}$ и подпространству $Y_{j}$ применяется лемма 1.26 .

Теорема 1.29 (теорема суммы). Если счетно паракомпактное пространство $X$ можно представить в виде обвединения счетного семейства замкнутых подмножеств $Y_{j}$, каждое из которых есть $w$-m-C-пространство, то $X$ является $w$-m-C-пространством.

Доказательство проходит по схеме доказательства теоремы 1.28. При этом дополнительно используются предложение 1.25 и лемма 1.27.2).

Теорема 1.30 (теорема монотонности). Всякое $F_{\sigma}$-подмножество счетно паракомпактного $w$-m-C-пространства является $w-m-C$-пространством.

Теорема 1.30 вытекает из теоремы 1.29 и наследования свойства $w-m-C$ замкнутыми подпространствами.

ЗАмечаниЕ 1.31. Как отмечено в замечании $1.4, C$-компакты могут содержать сильно бесконечномерные подмножества. Причем упомянутое там пространство $Y$ является $G_{\delta}$-подмножеством компакта Р. Поля. Таким образом, ни свойство $m-C$, ни свойство $w-m-C$ не наследуется $G_{\delta}$-подмножествами. В то же время, имеет место следующее предложение.

ПРЕДЛОЖЕНИЕ 1.32. Если всякое $G_{\delta}$-подмножество наследственно нормального пространства $X$ является $w$-m-C-пространством, то и всякое его подмножество является $w$-m-C-пространством.

ДокАЗАТЕЛЬство. Рассмотрим только случай $m$ - $C$-пространств. Пусть $Y \subset X$. Фиксируем $m$-последовательность $\left(k_{i}\right)$ и последовательность $\left(u_{i}\right)$ $k_{i}$-покрытий пространства $Y$. Каждое $u_{i}$ продолжаем до открытого в $X$ $k_{i}$-семейства $u_{i}^{1}$. Полагаем $Z=\bigcap_{i}\left(\bigcup u_{i}^{1}\right)$ и $u_{i}^{0}=u_{i}^{1} \mid Z$. По предположению в последовательность $\left(u_{i}^{0}\right) \quad k_{i}$-покрытий $G_{\delta}$-множества $Z$ можно $C$-вписать последовательность $\left(v_{i}^{0}\right)$ дизъюнктных открытых в $Z$ семейств. Полагая $v_{i}=v_{i}^{0} \mid Y$, получаем искомую последовательность $\left(v_{i}\right)$ дизъюнктных открытых в $Y$ семейств, $C$-вписанную в последовательность $\left(u_{i}\right)$. Предложение 1.32 доказано. 
ЗАмЕчАНИЕ 1.33. Наследственная нормальность пространства необходима для того, чтобы всякое его подпространство было $m$ - $C$-пространством.

Для $w-m-C$-пространств имеют место утверждения типа теоремы Даукера.

ПРЕДЛОЖЕНИЕ 1.34. Пусть подмножество $\left(F_{\sigma}\right.$-подмножество) $Y$ наследственно нормального (счетно паракомпактного) пространства $X$ является $w$-т-C-пространством и любое замкнутое в $X$ множество $F \subset X \backslash Y$ также является $w$-m-C-пространством. Тогда $w$-m-C-пространством является и все пространство $X$.

Как и теоремы сложения и суммы, доказывается предложение 1.34 на основе леммы 1.26 .

ЗАМЕчАНИЕ 1.35. Утверждения $1.28,1.29$ и 1.34 имеют место и для $m-C$ пространств (см. [15; §2]).

ЗАМЕчАНИЕ 1.36. Аналоги утверждений $1.28,1.29,1.30$ и 1.34 имеют место для $C$ - и $w$ - $C$-пространств. При этом неизбежно добавляется коллективная нормальность рассматриваемых пространств, без которой свойство $w-C$ не выполняется вообще. Теорема сложения для $C$-пространств доказана в [10], теорема суммы и теорема Даукера - в [15]. Докажем для иллюстрации одно из этих утверждений для $w$ - $C$-пространств.

ПРЕДЛОЖЕНИЕ 1.37 (теорема суммы). Если коллективно нормальное счетно паракомпактное пространство $X$ можно представить в виде обгединения замкнутых подмножеств $Y_{j} \in w-C, j \in \omega$, mо $X \in w-C$.

ДокАЗАТЕльство. Фиксируем последовательность $\left(\Phi_{i}\right), i \in \omega$, дискретных замкнутых семейств $\Phi_{i}=\left\{F_{\alpha}^{i}: \alpha \in A_{i}\right\}$. В силу коллективной нормальности $X$ существуют дискретные окрестности $O_{1} \Phi_{i}=\left\{O_{1} F_{\alpha}^{i}: \alpha \in A_{i}\right\}$ этих семейств. Представим $\omega$ в виде дизъюнктной суммы бесконечных множеств $N_{j}, j \in \omega$. Для $i \in N_{j}, \alpha \in A_{i}$ положим

$$
\Gamma_{\alpha}^{i}=\left[O_{1} F_{\alpha}^{i}\right] \cap Y_{j}
$$

и $\gamma_{i}=\left\{\Gamma_{\alpha}^{i}: \alpha \in A_{i}, i \in N_{j}\right\}$. Каждое семейство $\gamma_{i}$ дискретно. Поскольку $Y_{j} \in w-C$, существуют такие окрестности $O \gamma_{i}=\left\{O \Gamma_{\alpha}^{i}: \alpha \in A_{i}, i \in N_{j}\right\}$ семейств $\gamma_{i}$ в $Y_{j}$, что

$$
Y_{j}=\bigcup\left\{O \gamma_{i}: i \in N_{j}\right\}
$$

Положим $G_{i}=\bigcup O \gamma_{i}$. Согласно (1.9), семейство $\left\{G_{i}: i \in N_{j}\right\}$ является открытым покрытием счетно паракомпактного пространства $Y_{j}$. Его можно ужать до замкнутого покрытия $\left\{H_{i}: i \in N_{j}\right\}$. При этом без ограничения общности можно считать, что

$$
\bigcup\left\{\Gamma_{\alpha}^{i}: \alpha \in A_{i}\right\} \subset H_{i}
$$

Положим $H_{\alpha}^{i}=H_{i} \cap O \Gamma_{\alpha}^{i}, \alpha \in A_{i}$. В силу включения $H_{i} \subset G_{i}$ и дизъюнктности семейства $O \gamma_{i}$ множества $H_{\alpha}^{i}$ замкнуты, семейство $h_{i}=\left\{H_{\alpha}^{i}: \alpha \in A_{i}\right\}$ дискретно и

$$
\bigcup h_{i}=H_{i}
$$


Поскольку $X$ коллективно нормально, множества $H_{\alpha}^{i}$ можно заключить в попарно непересекающиеся открытые в $X$ множества $U_{\alpha}^{i}$. Полагаем

$$
O F_{\alpha}^{i}=O_{1} F_{\alpha}^{i} \cup U_{\alpha}^{i} \backslash \bigcup\left\{\left[O_{1} F_{\alpha^{\prime}}^{i}\right]: \alpha^{\prime} \neq \alpha\right\} .
$$

Из дизъюнктности семейств $O_{1} \Phi_{i}$ и $\left\{U_{\alpha}^{i}: \alpha \in A\right\}$ вытекает дизъюнктность семейства $O \Phi_{i}=\left\{O F_{\alpha}^{i}: \alpha \in A\right\}$. Поскольку $Y_{j}=\bigcup\left\{H_{i}: i \in N_{j}\right\}$, из (1.11) вытекает, что семейство $\left\{O \Phi_{i}: i \in N_{j}\right\}$ покрывает множество $Y_{j}$. Следовательно, $\left(\bigcup\left\{O \Phi_{i}: i \in \omega\right\}\right) \supset \bigcup Y_{j}=X$. Предложение 1.37 доказано.

Приведем некоторые следствия из теорем сложения и суммы. Скажем, что пространство $\sigma$-конечномерно, если оно является объединением счетного числа своих замкнутых конечномерных подпространств (в русской литературе такие пространства называются слабо счетномерными, в западной - сильно счетномерными ).

Из теоремы Остранда следует

ПРЕДЛОЖЕНИЕ 1.38 [15]. Всякое конечномерное пространство является $\infty$-C-пространством.

Из предложения 1.38 и теорем сложения и суммы для $m-C$-пространств вытекают соответственно

ПрЕДЛОЖЕНИЕ 1.39 [15]. Всякое счетномерное наследственно нормальное пространство является $\infty$-C-пространством.

ПРЕДЛОЖЕНИЕ 1.40 [15]. Всякое $\sigma$-конечномерное счетно паракомпактное пространство является $\infty$-C-пространством.

Эти утверждения вместе с предложениями 1.10 и 1.13 имеют естественные следствия для классов $w-m-C \supset m-C \supset \infty-C$.

Мы знаем (предложение 1.7), что паракомпактное конечномерное пространство является $C$-пространством. Насколько здесь можно ослабить паракомпактность?

Вопрос 5. Будет ли $C$-пространством всякое слабо паракомпактное конечномерное пространство?

Мы знаем также, что нульмерные пространства не обязаны быть $C$-пространствами. В то же время очевидным является следующее предложение.

ПрЕДЛОЖЕНИЕ 1.41. Всякое колективно нормалъное нулъмерное пространство является $w$-C-пространством.

Это утверждение допускает естественное распространение на пространства более высоких размерностей. Назовем нормальное пространство нуль-счетномерным, если его можно представить в виде объединения счетного числа нульмерных подпространств. Известно, что всякое счетномерное метризуемое пространство нуль-счетномерно. В то же время, не всякий конечномерный бикомпакт нуль-счетномерен (см. [35]).

ПреДЛОЖЕНИЕ 1.42. Всякое нуль-счетномерное коллективно и наследственно нормальное пространство является $w$-C-пространством. 
Доказательство. Пусть $X=\bigcup\left\{X_{i}: i \in \omega\right\}$, где $\operatorname{dim} X_{i}=0$. Берем в пространстве $X$ последовательность $\Phi_{i}=\left\{F_{\alpha}^{i}: \alpha \in A\right\}, i \in \omega$, дискретных замкнутых семейств. Поскольку $X$ коллективно и наследственно нормально, существуют такие дискретные семейства $u_{i}=\left\{U_{\alpha}^{i}: \alpha \in A\right\}$ окрестностей множеств $F_{\alpha}^{i}$, что

$$
X_{i} \cap \operatorname{Bd} U_{\alpha}^{i}=\varnothing .
$$

Фиксируем элемент $\alpha_{0}^{i} \in A_{i}$ и полагаем $O F_{\alpha_{0}^{i}}^{i}=X \backslash \bigcup\left\{\left[U_{\alpha}^{i}\right]: \alpha \neq \alpha_{0}^{i}\right\}$. Из-за дискретности семейства $u_{i}$ множество $O F_{\alpha_{0}^{i}}^{i}$ открыто. Полагаем также

$$
O F_{\alpha}^{i}=U_{\alpha}^{i}, \quad \text { если } \quad \alpha \neq \alpha_{0}^{i},
$$

и $\Phi_{i}=\left\{O F_{\alpha}^{i}: \alpha \in A_{i}\right\}$. Семейство $O \Phi_{i}$ является окрестностью семейства $\Phi_{i}$, которая согласно (1.12) покрывает множество $X_{i}$. Следовательно, $\bigcup\left\{O \Phi_{i}\right.$ : $i \in \omega\}$ - покрытие пространства $X$. Предложение 1.42 доказано.

СлеДСТВИЕ 1.43. Всякое подмножество линейно упорядоченного континуума является $w$-C-пространством.

В самом деле, всякое такое пространство наследственно коллективно нормально [36] и представляется в виде суммы двух нульмерных множеств.

Вопрос 6. Будет ли $w$ - $C$-пространством всякое коллективно нормальное конечномерное пространство?

Теорема 1.44 (локально конечной суммы для $C$-пространств, [15]). Ecлu счетно паракомпактное пространство $X$ может быть представлено в виде объединения локально конечного семейства замкнутых множеств $F_{\alpha} \in C$, то $X \in C$.

Теорема 1.45 (локально конечной суммы для $w$ - $C$-пространств). Eсли счетно паракомпактное коллективно нормальное пространство $X$ может быть представлено в виде объединения локально конечного семейства замкнутых множеств $F_{\alpha} \in w-C$, mо $X \in w-C$.

ДокАзАтЕльство. Для натурального $k$ обозначим через $Z_{k}$ множество всех точек $x \in X$, принадлежащих по крайней мере $k$ элементам семейства $\Phi=\left\{F_{\alpha}\right.$ : $\alpha \in A\}$. В силу локальной конечности этого семейства множества $Z_{k}$ замкнуты, а их пересечение пусто. Следовательно, открытые множества $G_{k}=X \backslash Z_{k+1}$ образуют покрытие. Поскольку $X$ счетно паракомпактно, существуют замкнутые множества $\Gamma_{k} \subset G_{k}$, дающие в объединении все $X$. Поэтому в силу предложения 1.37 достаточно показать, что каждое множество $\Gamma_{k}$ является $w$ - $C$ пространством. А для этого достаточно доказать нашу теорему в предположении ограниченной кратности семейства $\Phi$.

Делается это индукцией по кратности $n$. Если $n=1$, то локально конечное семейство $\Phi$ дискретно, а для дискретного семейства теорема суммы выполнена очевидным образом. Сделаем индуктивный переход от $n$ к $n+1$. Обозначим через $A(n+1)$ множество всех подмножеств $\left\{\alpha_{1}, \ldots, \alpha_{n+1}\right\} \subset A$, состоящих из $n+1$ различных элементов, т.е. $A(n+1)=\exp _{n+1} A \backslash \exp _{n} A$. Для каждого $a=\left\{\alpha_{1}, \ldots, \alpha_{n+1}\right\} \in A(n+1)$ положим $F_{a}=F_{\alpha_{1}} \cap \cdots \cap F_{\alpha_{n+1}}$. Если элементы 
$a, a^{\prime} \in A(n+1)$ различны, то множества $F_{a}$ и $F_{a^{\prime}}$ не пересекаются. Кроме того, очевидно, что семейство $\left\{F_{a}: a \in A(n+1)\right\}$ локально конечно и, следовательно, дискретно. Поскольку $X$ коллективно нормально, существуют такие окрестности $O F_{a}$, что семейство $\left\{\left[O F_{a}\right]: a \in A(n+1)\right\}$ дискретно. Более того, если $a=\left\{\alpha_{1}, \ldots, \alpha_{n+1}\right\}$, то можно считать, что $\left[O F_{a}\right] \subset F_{\alpha_{1}} \cup \cdots \cup F_{\alpha_{n+1}}$, так как последнее множество содержит окрестность множества $F_{a}$. Поэтому каждое множество $\left[O F_{a}\right]$ является $w$ - $C$-пространством по теореме конечной суммы, а $Z=\bigcup\left\{\left[O F_{a}\right]: a \in A(n+1)\right\} \in w-C$ - по теореме дискретной суммы. Полагаем $Y=X \backslash \bigcup\left\{O F_{a}: a \in A(n+1)\right\}$. Множество $Y$ замкнуто, а семейство $\Phi \mid Y$ имеет кратность $\leqslant n$. Значит, $Y \in w-C$ по предположению индукции. Следовательно, $X=Y \cup Z \in w-C$. Теорема 1.45 доказана.

Теорема 1.46 (локально конечной суммы для $m$ - $C$-пространств, $[15]) . E c$ ли счетно паракомпактное (или наследственно нормальное) пространство $X$ может быть представлено в виде объединения локально конечного семейства замкнутых множеств $F_{\alpha} \in m-C$, mо $X \in m-C$.

ТЕОРема 1.47 (локально конечной суммы для $w$-m-C-пространств). Ecлu счетно паракомпактное (или наследственно нормальное) пространство $X$ может быть представлено в виде обгединения локально конечного семейства замкнутых множеств $F_{\alpha} \in w-m-C$, то $X \in w-m-C$.

Для счетного паракомпактного коллективно нормального $X$ доказательство повторяет доказательство теоремы 1.45. В общем случае надо воспользоваться схемой доказательства теоремы 6.1.15 локально конечной суммы для слабо бесконечномерных пространств из [18].

ЗАмЕчАНИЕ 1.48. В силу совпадения классов $2-C=w-2-C=$ wid, теоремы 1.46 и 1.47 обобщают соответствующую теорему суммы для слабо бесконечномерных пространств, доказанную Н. Хаджиивановым [37] для счетно паракомпактных и Л. Полковским [38] для наследственно нормальных пространств.

В дальнейшем нам понадобится следующее утверждение, являющееся аналогом леммы 1.26 для $C$-пространств.

Лемма 1.49 [15]. Пусть счетно паракомпактное коллективно нормальное пространство $X$ содержит замкнутое множество $F \in C$. Тогда для всякой последовательности $u_{i}, i \in \omega$, открытых покрытий пространства $X$ существует такая последовательность $v_{i}$ дизгюнктных открытых в $X$ семейств, что $v_{i}$ вписано в $u_{i}$ и семейство $\bigcup\left\{v_{i}: i \in \omega\right\}$ покрывает множество $F$.

Следующее утверждение дополняет теорему 1.8, указывая еще один широкий класс $C$-пространств.

Теорема 1.50. Всякое сильно паракомпактное пространство $X$, малая трансбинитная размерность ind $X$ которого определена, является $C$-пространством.

ДокАЗАТЕЛЬСтво. Индукция по ind $X$. Если ind $X=0$, то по теореме Мориты [39] $\operatorname{dim} X=0$. Следовательно, $X \in C$ по предложению 1.7. 
Пусть теперь ind $X=\alpha>0$. Фиксируем последовательность покрытий $u_{i}$, $i \in \omega$, из определения 1.2. В покрытие $u_{0}$ можно вписать такое покрытие $\Omega=\{O x: x \in X\}$, что

$$
\text { ind } \operatorname{Bd} O x<\alpha \text {. }
$$

Существует звездно-конечное покрытие $w$, вписанное в покрытие $\Omega$. Оно распадается в дизъюнктную сумму счетных семейств $w_{\beta}, \beta \in B$, тела $W_{\beta}$ которых попарно не пересекаются и, следовательно, открыто-замкнуты. Для доказательства теоремы, согласно лемме 1.49 , достаточно в покрытие $u_{0} \mid W_{\beta}$ множества $W_{\beta}$ вписать такое дизъюнктное открытое семейство $v_{\beta}$, что

$$
W_{\beta} \backslash \bigcup v_{\beta} \in C
$$

Пусть $w_{\beta}=\left\{W_{j}^{\beta}: j \in \omega\right\}$. Поскольку $w$ вписано в $\Omega$, для каждого $j$ существует такая точка $x_{j}^{\beta}$, что $W_{j}^{\beta} \subset O x_{j}^{\beta}$. Положим $V_{0}^{\beta}=O x_{0}^{\beta} \cap W_{\beta}$ и $V_{j}^{\beta}=\left(O x_{j}^{\beta} \backslash\right.$ $\left.\bigcup\left\{\left[O x_{k}^{\beta}\right]: k<j\right\}\right) \cap W_{\beta}$ при $j \geqslant 1$. Семейство $v_{\beta}=\left\{V_{j}^{\beta}: j \in \omega\right\}$ дизъюнктно, вписано в $u_{0} \mid W_{\beta}$ и

$$
W_{\beta} \backslash \bigcup v_{\beta} \subset \bigcup\left\{\operatorname{Bd} O x_{j}^{\beta}: j \in \omega\right\}
$$

Но из (1.13) по предположению индукции следует, что $\operatorname{Bd} O x_{j}^{\beta} \in C$. Поэтому свойство (1.14) вытекает из (1.15) и теоремы счетной суммы. Теорема 1.50 доказана.

Эта теорема уточняет аналогичную теорему Б. Т. Левшенко [40], доказавшего, что $X \in$ wid.

Вопрос 7. Будет ли слабо бесконечномерным ( $C$-пространством $)$ всякое паракомпактное пространство, малая трансфинитная размерность ind которого определена?

\section{Усилением вопроса 7 является}

Вопрос 8. Будет ли слабо бесконечномерным всякое метрическое пространство $X$ размерности ind $X=0$.

Вопрос 7 можно усилить и в другом направлении. Для этого напомним, что регулярное пространство $X$ называется вполне паракомпактным [41], если для всякого открытого покрытия $u$ пространства $X$ существует последовательность $v_{i}, i \in \omega$, звездно-конечных покрытий, объединение которых содержит покрытие, вписанное в $u$.

Вопрос 9. Будет ли слабо бесконечномерным всякое вполне паракомпактное пространство, малая трансфинитная размерность ind которого определена?

ЗАмечАниЕ 1.51. Аналога вопроса 8 для вполне паракомпактного случая не имеется. В самом деле, Ю. М. Смирнов доказал [42], что вполне паракомпактное метризуемое пространство, малая трансфинитная размерность ind которого определена, счетномерно и, следовательно, является $C$-пространством согласно теореме 1.8 . 
Напомним одно определение, введенное в [43]. Разгородкой замкнутых множеств $A$ и $B$ пространства $X$ называется такое замкнутое множество $C$, что существуют открытые множества $U \supset A \backslash B$ и $V \supset B \backslash A$, для которых

$$
X=U \sqcup C \sqcup V .
$$

Разгородка непересекающихся множеств является перегородкой между ними.

Нормальное пространство $X$ называется $W$-пространством $(X \in W)$, если для любой последовательности $\left(A_{i}, B_{i}\right), i \in \omega$, пар замкнутых подмножеств $X$ существуют разгородки $C_{j}$ множеств $A_{i}$ и $B_{i}$, удовлетворяющие условию

$$
\cap\left\{C_{i}: i \in \omega\right\} \subset \cup\left\{A_{i} \cap B_{i}: i \in \omega\right\} .
$$

Теорема 1.52 [43]. Всякое $W$-пространство слабо бесконечномерно. В классе наследственно нормальных пространств класс $W$ обладает следующими свойствами:

1) удовлетворяет теореме сложения;

2) наследуется при переходе к подпространству;

3) всякое счетномерное пространство является $W$-пространством.

ТЕОРема 1.53. Наследственно нормалъное пространство $X$ является $W$-пространством тогда и только тогда, когда оно наследственно слабо бесконечномерно.

ДокАЗАТЕЛьство. Необходимость вытекает из сказанного в теореме 1.52. Предположим теперь, что всякое подпространство наследственно нормального пространства $X$ слабо бесконечномерно. Покажем, что $X \in W$. Пусть $\left(A_{i}, B_{i}\right)$, $i \in \omega,-$ пары замкнутых подмножеств $X$. Положим

$$
A_{i}^{0}=A_{i} \backslash B_{i}, \quad B_{i}^{0}=B_{i} \backslash\left[A_{i}^{0}\right] .
$$

В силу наследственной нормальности $X$ существуют такие открытые множества $G_{i} \supset A_{i}^{0}$ и $H_{i} \supset B_{i}^{0}$, что

$$
\left[G_{i}\right] \cap\left[H_{i}\right] \subset\left[A_{i}^{0}\right] \cap\left[B_{i}^{0}\right] .
$$

Положим

$$
X_{0}=X \backslash \bigcup\left\{\left[A_{i}^{0}\right] \cap\left[B_{i}^{0}\right]: i \in \omega\right\}
$$

И

$$
K_{i}=\left[G_{i}\right] \cap X_{0}, \quad L_{i}=\left[H_{i}\right] \cap X_{0} .
$$

Согласно (1.17), замкнутые в $X_{0}$ множества $K_{i}$ и $L_{i}$ не пересекаются. Поскольку $X_{0}$ слабо бесконечномерно, существуют такие непересекающиеся открытые в $X_{0}$ множества $U_{i}^{0} \supset K_{i}$ и $V_{i}^{0} \supset K$, что

$$
X_{0}=\bigcup\left\{U_{i}^{0} \cup V_{i}^{0}: i \in \omega\right\} .
$$

А в силу наследственной нормальности пространства $X$ существуют непересекающиеся канонически открытые в $X$ множества $U_{i}$ и $V_{i}$, удовлетворяющие условию

$$
U_{i}^{0} \subset U_{i}, \quad V_{i}^{0} \subset V_{i} .
$$


Кроме того, увеличивая множества $V_{i}$, можно считать, что

$$
V_{i}=X \backslash\left[U_{i}\right] .
$$

Положим $C_{i}=X \backslash U_{i} \cup V_{i}$. Имеем $\bigcap\left\{C_{i}: i \in \omega\right\}=X \backslash \bigcup\left\{U_{i} \cup V_{i}: i \in\right.$ $\omega\} \stackrel{(1.20)}{\subset} X \backslash X_{0} \stackrel{(1.18)}{=} \bigcup\left\{\left[A_{i}^{0}\right] \cap\left[B_{i}^{0}\right]: i \in \omega\right\} \stackrel{(1.16)}{\subset} \bigcup\left\{A_{i} \cap B_{i}: i \in \omega\right\}$. Остается проверить, что $C_{i}$ являются разгородками множеств $A_{i}$ и $B_{i}$. Для этого достаточно установить, что $A_{i}^{0} \subset U_{i}, B_{i}^{0} \subset V_{i}$. Из (1.16) вытекает, что множество $X_{0}$ всюду плотно в $X$. Следовательно, для открытого множества $G_{i}$ имеем $\left[G_{i}\right]=\left[G_{i} \cap X_{0}\right]$. Поэтому из (1.19) получаем $\left[K_{i}\right]=\left[G_{i}\right]$. Таким образом,

$$
A_{i}^{0} \subset\left[G_{i}\right]=\left[K_{i}\right] \subset\left[U_{i}^{0}\right] \subset\left[U_{i}\right], \quad \text { т.е. } \quad A_{i}^{0} \subset G_{i} \subset\left[U_{i}\right] .
$$

Следовательно, $A_{i}^{0} \subset \operatorname{Int}\left[U_{i}\right]=U_{i}$. Аналогичным образом, $B_{i}^{0} \subset V_{i}$. Teорема 1.53 доказана.

\section{§ 2. Отображения, понижающие размерность}

ОПределЕниЕ 2.1. Скажем, что $f: X \rightarrow Y$ есть $C$-отображение $(f \in C)$, если $f^{-1}(y) \in C$ для каждой точки $y \in Y$. Аналогично определяются $w-C$-, $m-C-, w-m-C$-отображения.

Tеорема 2.2. Пусть $f: X \rightarrow Y$ - замкнутое отображение счетно паракомпактного (или наследственно нормального) пространства $X$ на C-nространство $Y$. Тогда:

1) если $f \in m-C$, то $X \in m-C$;

2) если $f \in w-m-C$, то $X \in w-m-C$.

Пункт 1) доказан в [15]. Доказательство пункта 2) проходит по схеме доказательства теоремы 6.3 .9 из [18], посвященной случаю $m=2$ (теоремы ХатториЯмады [44]).

Пусть $\Phi=\left\{F_{\alpha}: \alpha \in A\right\}-$ дискретное семейство подмножеств пространства $X$. Положим

$$
U_{\alpha}(\Phi)=X \backslash \bigcup\left\{F_{\beta} \in \Phi: \beta \neq \alpha\right\} \quad \text { и } \quad c(\Phi)=\left\{U_{\alpha}(\Phi): \alpha \in A\right\} .
$$

Семейство $c(\Phi)$ является открытым покрытием пространства $X$. Следующее утверждение было уже неявно использовано (см. предложение 1.16).

ПРЕДЛОЖЕНИЕ 2.3. Для того, чтобъ коллективно нормальное пространство $X$ было $w$-C-пространством, необходимо и достаточно выполнение следующего условия:

для любой последовательности $\left(\Phi_{i}\right), i \in \omega$, дискретных семейств замкнутых подмножеств $X$ существует последовательность $\left(v_{i}\right)$ дизгюнктных открытых семейств, комбинаторно $C$-вписанная в последовательность $\left(c\left(\Phi_{i}\right)\right)$.

Теорема 2.4. Пусть $f: X \rightarrow Y$ - замкнутое отображение счетно паракомпактного коллективно нормального (или наследственно коллективно нормального) пространства $X$ на $C$-пространство $Y$. Тогда: 
1) если $f \in C$, mo $X \in C$;

2) если $f \in w-C$, mо $X \in w-C$.

ДокАЗАТЕЛЬСтво проведем лишь для пункта 2) в случае наследственно коллективно нормального $X$. Пункт 1 ) для счетно паракомпактного $X$ доказан в [15]. Оставшиеся два варианта аналогичны упомянутым.

Итак, пусть $\Phi_{i}=\left\{F_{\alpha}^{i}: \alpha \in A_{i}\right\}, i \in \omega,-$ последовательность дискретных замкнутых в $X$ семейств. Множества $U_{\alpha}\left(\Phi_{i}\right)$ из $(2.1)$ обозначим через $U_{\alpha}^{i}$. Представим множество $\omega$ в виде дизъюнктной суммы бесконечномерных множеств $N_{j}, j \in \omega$. Поскольку $f \in w$ - , для каждой точки $y \in Y$ согласно предложению 2.3 существует такая последовательность $v_{i, y}^{0}=\left\{{ }^{0} V_{\alpha}^{i, y}: \alpha \in A_{i}\right\}, i \in$ $\omega$, дизъюнктных открытых в $f^{-1}(y)$ семейств, что каждая подпоследовательность $v_{i, y}^{0}, i \in N_{j}$, комбинаторно $C$-вписана в последовательность $c\left(\Phi_{i}\right) \mid f^{-1}(y)$, $i \in N_{j}$. В силу наследственной коллективной нормальности $X$ каждое семейство $v_{i, y}^{0}$ можно продолжить до дизъюнктного открытого в $X$ семейства $v_{i, y}=\left\{V_{\alpha}^{i, y}: \alpha \in A_{i}\right\}$. При этом, согласно сказанному выше, можно считать, что

$$
\begin{gathered}
V_{\alpha}^{i, y} \subset U_{\alpha}^{i}, \\
f^{-1}(y) \subset \bigcup\left\{V_{\alpha}^{i, y}: i \in N_{j}, \alpha \in A_{i}\right\}, \quad j \in \omega .
\end{gathered}
$$

Положим

$$
V_{j, y}=\bigcup\left\{V_{\alpha}^{i, y}: i \in N_{j}, \alpha \in A_{i}\right\}, \quad j \in \omega .
$$

Из (2.3) и (2.4) следует

$$
f^{-1}(y) \subset V_{j, y}, \quad j \in \omega .
$$

Поэтому из замкнутости отображения $f$ вытекает существование такой окрестности $G_{j, y}$ точки $y$, что

$$
f^{-1}\left(G_{j, y}\right) \subset V_{j, y}, \quad j \in \omega .
$$

Поскольку $y \in C$, существует такая последовательность $\left(h_{j}\right)$ дизъюнктных открытых в $Y$ семейств, что

$$
\begin{aligned}
& h_{j} \text { вписано в покрытие } g_{j}, j \in \omega ; \\
& \bigcup\left\{h_{j}: j \in \omega\right\} \text { является покрытием } Y \text {. }
\end{aligned}
$$

Уточняя (2.7), для каждого $H \in h_{j}$ зафиксируем такую точку $y \in y(H)$, что

$$
H \subset G_{j, y(H)}
$$

Для каждого $i \in N_{j}$ и $\alpha \in A_{i}$ положим

$$
W_{\alpha}^{i}=\bigcup\left\{f^{-1}(H) \cap V_{\alpha}^{i, y(H)}: H \in h_{j}\right\} .
$$

Свойство (2.2) влечет

$$
W_{\alpha}^{i} \subset U_{\alpha}^{i}
$$


Из дизъюнктности семейств $v_{i, y}$ и $h_{j}$ вытекает дизъюнктность семейств $w_{i}=$ $\left\{W_{\alpha}^{i}: \alpha \in A_{i}\right\}$. С учетом (2.11) и предложения 2.3 остается показать, что семейство $\bigcup\left\{w_{i}: i \in \omega\right\}$ покрывает $X$. Для этого, в силу (2.8), достаточно проверить, что

$$
f^{-1}(H) \subset \bigcup\left\{W_{\alpha}^{i}: i \in N_{j}, \alpha \in A_{i}\right\} \quad \text { для всякого } H \in h_{j} .
$$

Имеем

$$
f^{-1}(H) \stackrel{(2.9)}{\subset} f^{-1}\left(G_{j, y(H)}\right) \stackrel{(2.6)}{\subset} V_{j, y(H)}
$$

Следовательно,

$$
\begin{aligned}
f^{-1}(H) & \subset f^{-1}(H) \cap V_{i, y(H)} \stackrel{(2.4)}{=} \bigcup\left\{f^{-1}(H) \cap V_{\alpha}^{i, y(H)}: i \in N_{j}, \alpha \in A_{i}\right\} \\
& \stackrel{(2.10)}{\subset} \bigcup\left\{W_{\alpha}^{i}: i \in N_{j}, \alpha \in A_{i}\right\} .
\end{aligned}
$$

Теорема 2.4 доказана.

ОПРЕДЕЛЕНиЕ 2.5 [15]. Пусть $\mathscr{P}$ - некоторый класс пространств, содержащий все замкнутые подпространства своих элементов. Замкнутое отображение

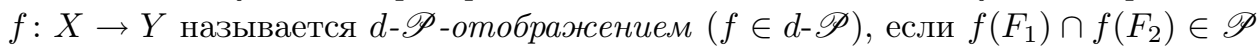
для любых непересекающихся замкнутых множеств $F_{1}, F_{2} \subset X$. В частности, при $\mathscr{P}=C$ получаем определение $d$-C-отображения.

Если $\mathscr{P}$ - некоторый класс пространств, то через $\sigma \mathscr{P}$ обозначим класс всех $F_{\sigma}$-подмножеств пространств из класса $\mathscr{P}$.

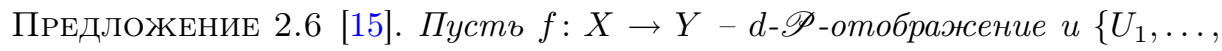
$\left.U_{k}\right\}$ - конечное функционально открытое покрытие пространства $X$. Тогда

$$
A \equiv Y \backslash f^{\#} U_{1} \cup \cdots \cup f^{\#} U_{k} \in \sigma \mathscr{P} .
$$

Теорема 2.7. Пусть $f: X \rightarrow Y-d$-C-отображение счетно паракомпактного или наследственно нормального пространства $X$ на счетно паракомпактное коллективно нормальное пространство $Y$. Тогда

1) если $Y \in m-C$ u $f \in m-C, m o X \in m-C$;

2) если $Y \in w-m-C$ u $f \in w-m-C$, mo $X \in w-m-C$;

3) если $Y \in w-C$ u $f \in w-C$, mo $X \in w-C$.

ДокАзАтЕльство. Пункт 1) представляет собой теорему 5.7 из [15]. Рассмотрим пункт 2) для счетно паракомпактного $X$. Пусть

$$
\Phi_{i}=\left\{F_{1}^{i}, \ldots, F_{k_{i}}^{i}\right\}, \quad i \in \omega
$$

- последовательность $m$-систем пространства $X$. Без ограничения общности можно считать, что множества $F_{j}^{i}$ функционально замкнуты и, следовательно, множества $U_{j}^{i}=X \backslash \bigcup\left\{F_{l}^{i}: l \neq j\right\}$ функционально открыты. Положим

$$
B_{i}=Y \backslash \bigcup\left\{f^{\#} U_{j}^{i}: 1 \leqslant j \leqslant k_{i}\right\} .
$$

В силу предложения 2.6 $B_{i} \in \sigma C$, а по теореме счетной суммы для $C$-пространств (см. замечание 1.36 ) $B_{i} \in C$. Согласно теореме 2.2 , множество 
$F_{i}=f^{-1}\left(B_{i}\right)$ есть $w$-m- $C$-пространство. Ввиду леммы 1.26 существуют такие окрестности $O \Phi_{i}$ систем $\Phi_{i}$, что семейство $\Omega=\bigcup\left\{O \Phi_{i}: i\right.$ четно $\}$ покрывает множество $\bigcup\left\{F_{i}: i \in \omega\right\}$. В силу замкнутости $f$ существует такое открытое множество $\Gamma \subset Y$, что

$$
\bigcup\left\{F_{i}: i \in \omega\right\} \subset f^{-1}(\Gamma) \subset \bigcup \Omega .
$$

Положим $Z=Y \backslash \Gamma$ и $L_{j}^{i}=Z \cap f\left(F_{j}^{i}\right)$. Каждое семейство $\lambda_{i}=\left\{L_{1}^{i}, \ldots, L_{k_{i}}^{i}\right\}$ является $m$-системой. Поскольку $Z \in m$ - $C$, можно найти такие замкнутые множества $M_{j}^{i} \supset L_{j}^{i}$, что $M_{j_{1}}^{i} \cap M_{j_{2}}^{i}=\varnothing$ при $j_{1} \neq j_{2}$ и

$$
\bigcup\left\{M_{j}^{i}: 1 \leqslant j \leqslant k_{i}, i \text { четно }\right\}=Z \text {. }
$$

Положим $H_{j}^{i}=F_{j}^{i} \cup f^{-1}\left(M_{j}^{i}\right)$. Для каждого $i$ семейство $h_{i}=\left\{H_{1}^{i}, \ldots, H_{k_{i}}^{i}\right\}$ дизъюнктно и является укрупнением системы $\Phi_{i}$. Для четного $i$ берем в качестве $O \Phi_{i}$ произвольную окрестность $O h_{i}$ системы $h_{i}$. Из (2.13) и (2.14) вытекает, что $\left\{O \Phi_{i}: i \in \omega\right\}$ - искомая последовательность окрестностей систем $\Phi_{i}$. Остальные случаи рассматриваются аналогично. Теорема 2.7 доказана.

Для приложений теоремы 2.7 надо напомнить некоторые понятия и факты.

ОПредЕЛЕНиЕ 2.8 [15]. Замкнутое отображение $f: X \rightarrow Y$ называется $d$-разреженным, если для любых непересекающихся замкнутых множеств $F_{1}, F_{2} \subset$ $X$ множество $f\left(F_{1}\right) \cap f\left(F_{2}\right)$ разрежено, т.е. всякое его непустое подпространство имеет изолированную точку.

Всякое вполне замкнутое отображение (см. [45]) $d$-разрежено, поскольку для вполне замкнутого отображения $f$ множество $f\left(F_{1}\right) \cap f\left(F_{2}\right)$ из определения 2.8 дискретно.

ТЕОРЕма 2.9 [15]. Всякое слабо паракомпактное разреженное пространство нульмерно.

Поскольку всякое нульмерное паракомпактное пространство есть $C$-пространство, из теоремы 2.9 вытекает следующее предложение.

ПрЕДЛОЖЕНИЕ 2.10. Всякое $d$-разреженное отображение на паракомпактное пространство является $d$-C-отображением.

Обладая хорошими размерностными свойствами, класс $d$-разреженных отображений вместе с тем достаточно широк. Композиция совершенных $d$-разреженных отображений $d$-разрежена [15]. Поэтому $d$-разреженным будет не только всякое вполне замкнутое отображение, но и всякая композиция вполне замкнутых совершенных отображений. Более того, имеет место следующее предложение.

ПРЕДЛОЖЕНИЕ 2.11 [15]. Пусть $S=\left\{X_{\alpha}, \pi_{\beta}^{\alpha}, A\right\}-$ такой непрерывный спектр из бикомпактов, что все соседние проекции $\pi_{\alpha}^{\alpha+1} d$-разрежены. Тогда все его проекиии d-разрежены.

СлЕДСТВИЕ 2.12. Если все соседние проекции непрерывного спектра из бикомпактов вполне замкнуты, то все его проекиии d-разрежены. 
Из теоремы 2.7 и предложения 2.10 вытекает следующая теорема.

Теорема 2.13. Предположим, что счетное паракомпактное или наследственно нормальное пространство $X$ допускает $d$-разреженное отображение на паракомпактное пространство $Y$. Тогда

1) если $Y \in m-C$ u $f \in m-C, m o X \in m-C$;

2) если $Y \in w-m-C$ u $f \in w-m-C$, mo $X \in w-m-C$;

3) если $Y \in w-C$ u $f \in w-C$, mo $X \in w-C$.

Теорема 2.14. Пусть $S=\left\{X_{\alpha}, \pi_{\beta}^{\alpha}, A\right\}$ - обратный спектр, состоящий из бикомпактов и d-разреженных отображений, и пусть $X=\lim S$. Тогда

1) если все $X_{\alpha} \in m-C$, то $X \in m-C$;

2) если все $X_{\alpha} \in w-m-C$, то $X \in w-m-C$.

ДокАзАтельСтво. Надо разобрать только пункт 2), поскольку 1) - это теорема 5.16 из [15]. Фиксируем последовательность

$$
\Phi_{i}=\left\{F_{1}^{i}, \ldots, F_{k_{i}}^{i}\right\}, \quad i \in \omega
$$

$m$-систем бикомпакта $X$. Существует такое $\alpha \in A$, что семейство $\pi_{\alpha}\left(F_{1}^{0}\right), \ldots$, $\pi_{\alpha}\left(F_{k_{i}}^{0}\right)$, где $\pi_{\alpha}: X \rightarrow X_{\alpha}$ - предельная проекция спектра, дизъюнктно. Отображение $\pi_{\alpha}$, очевидно, $d$-разрежено. Поэтому для каждого $i \geqslant 1$ множество

$$
B_{i}=\bigcup\left\{\pi_{\alpha}\left(F_{j_{1}}^{i}\right) \cap \pi_{\alpha}\left(F_{j_{2}}^{i}\right): j_{1} \neq j_{2}\right\}
$$

разрежено. Следовательно, множество $B=\bigcup\left\{B_{i}: i \geqslant 1\right\}$ нульмерно и финально компактно. Тогда существует такое дизъюнктное семейство окрестностей $O \pi_{\alpha}\left(F_{1}^{o}\right), \ldots, O \pi_{\alpha}\left(F_{k_{1}}^{o}\right)$, что

$$
B \subset \bigcup\left\{O \pi_{\alpha}\left(F_{j}^{0}\right): 1 \leqslant j \leqslant k_{1}\right\} .
$$

Положим $Z=X_{\alpha} \backslash \bigcup\left\{O \pi_{\alpha}\left(F_{j}^{o}\right): 1 \leqslant j \leqslant k_{1}\right\}$ и $L_{j}^{i}=\pi_{\alpha}\left(F_{j}^{i}\right)$. Далее, применяя рассуждения из доказательства п. 2) теоремы 2.7, завершаем доказательство теоремы 2.14 .

Tеорема 2.15. Пусть $S=\left\{X_{\alpha}, \pi_{\beta}^{\alpha}, A\right\}$ - непрерывный спектр из бикомпактов, все соседние проекиии которого d-разрежсены и являются $m$ - $C$ $(w-m-C)$-отображениями. Тогда если $X_{0} \in m-C(w-m-C), m o X=\lim S \in m-C$ $(w-m-C)$.

ДокАЗАТЕЛЬСтво. По предложению 2.11 все проекции $\pi_{\beta}^{\alpha}$ - $d$-разрежены. Поэтому, согласно теореме 2.14, достаточно показать, что все $X_{\alpha} \in m$ - $C$ $(w-m-C)$. Делается это индукцией по $\alpha$. Переход от $\alpha$ к $\alpha+1$ происходит с применением теоремы 2.7, при переходе к предельному $\alpha$ применяется теорема 2.14.

СлеДСтвиЕ 2.16. Пусть $S=\left\{X_{\alpha}, \pi_{\beta}^{\alpha}, A\right\}$ - непрерывный спектр из бикомпактов, все соседние проекции которого вполне замкнуты и слабо бесконечномерны. Тогда если бикомпакт $X_{0}$ слабо бесконечномерен, то и предел спектра $S$ слабо бесконечномерен.

Это утверждение дает положительный ответ на вопрос из [35]. 


\section{§ 3. Отображения, повышающие размерность}

Теорема 3.1. Пусть $f: X \rightarrow Y$ - такое отображение бикомпакта $X$ на $Y$, что $\left|\varphi^{-1}(y)\right|<\mathbf{c}$ для каждой точки $y \in Y$. Тогда:

1) если $X \in m-C$, то $Y \in m-C$;

2) если $X \in w-m-C$, mo $Y \in w-m-C$.

Случай 1) расмотрен в [15; теорема 6.2]. Доказательство в случае 2) проходит по аналогичной схеме с применением нижеследующей леммы 3.2. Для $m=2$ теорема доказана Е. Г. Скляренко в [46].

Лемма 3.2. Пусть $f: X \rightarrow Y$ - замкнутое отображение счетно паракомпактного $w$-m-C-пространства $X$ на $Y$. Если $Y$ не является $w-m-C$-пространством, то существуют такие непересекающиеся замкнутые множества $X_{0}, X_{1} \subset X$, что $f\left(X_{0}\right)=f\left(X_{1}\right)$ u $f\left(X_{0}\right) \notin w-m-C$.

Доказательство во многом повторяет доказательство соответствующей леммы 6.1 из [15] для $m$ - $C$-пространств (см. также лемму 6.3 .3 из [18]).

Из теоремы 3.1 при $m=\infty$ вытекает следующее утверждение.

СледСтвиЕ 3.3. Счетнократный образ $C(w-C)$-бикомпакта является $C$ $(w-C)$-бикомпактом.

Tеорема 3.4. Пусть $f: X \rightarrow Y$ - замкнутое отображение конечной кратности $\leqslant n$ счетно паракомпактного пространства $X$ на $Y$. Тогда если $X \in$ $m-C(X \in w-m-C)$, mo $Y \in m-C(Y \in w-m-C)$.

Случай $m$ - $C$-пространств рассмотрен в [15; теорема 6.5]. По аналогичной схеме с применением леммы 3.2 проходит доказательство для $w-m$ - $C$-пространств. Для $m=2$ теорема доказана Л. Полковским [38].

Теорема 3.5. Пусть $f: X \rightarrow Y$ - замкнутая сюргекция конечной кратноcmu $\leqslant n$. Тогда:

1) если $X$ - паракомпактное $C$-пространство, то $Y \in C$;

2) если $X$ - коллективно нормальное $w$-C-пространство, то $Y \in w-C$.

Для $C$-пространств утверждение доказано в [15; теорема 6.8]. В случае $w$ - $C$-пространств применяется следующая лемма.

Лемма 3.6. Пусть $f: X \rightarrow Y$ - замкнутое отображение коллективно нормального $w$-C-пространства $X$ на $Y$. Если $Y$ не является $w$-C-пространством, то существуют такие непересекающиеся замкнутые множества $X_{0}, X_{1} \subset X$, umo $f\left(X_{0}\right)=f\left(X_{1}\right)$ u $f\left(X_{0}\right) \notin w-C$.

Теорема 3.7. Пусть $f: X \rightarrow Y$ - такое замкнутое отображение метризуемого пространства $X$ на метризуемое пространство $Y$, что каждый слой $f^{-1}(y)$ либо имеет изолированную точку, либо является множеством мощности < с. Тогда

1) $X \in m-C \Rightarrow Y \in m-C$;

2) $X \in w-m-C \Rightarrow Y \in w-m-C$. 
Случай $m$ - $C$-пространств расмотрен в [15; теорема 6.17$].$ Для $w$ - $m$ - $C$-пространств доказательство проходит по аналогичной схеме (см. также [18; теорема 6.3.2]). Теорему 3.7 для $m=2$ доказал В. Ольшевский (см. [18; теорема 6.3.2]). Первый результат в этом направлении получил Е. Г. Скляренко [46] (пространство $X$ вполне метризуемо, слои $f^{-1}(y)$ имеют мощность $<\mathbf{c}$ ).

Tеорема 3.8 [15]. Ecли $f: X \rightarrow Y$ есть $d$-m-C-отображение счетно паракомпактного $m$-C-пространства $X$ на $Y$, то $Y \in m-C$.

Из теорем 3.8 и 2.9 вытекает

СлЕДСтвиЕ 3.9. Если $f: X \rightarrow Y$ - сюргективное $d$-разреженное отображение паракомпактного $m$-C-пространства, то $Y \in m-C$.

Поскольку всякое вполне замкнутое отображение $d$-разрежено, из теоремы 3.8 получаем

СлЕДСТвИЕ 3.10. Если $f: X \rightarrow Y$ - сюргективное вполне замкнутое отображение счетно паракомпактного $m$-C-пространства, то $Y \in m$-C.

ЗАмЕчАНИЕ 3.11. Аналоги теоремы 3.8 и следствий 3.9 и 3.10 имеют место для $w-m$ - $C$-пространств и $d-w-m-C$-отображенией.

\section{§4. Трансфинитные продолжения лебеговой размерности и размерность $\operatorname{Ind}_{m}$}

Теорема 4.1. Для нормального пространства $X$ u $m \in \overline{\mathbb{N}}$ следующие условия равносильны:

1) $\operatorname{dim} X \leqslant n$;

2) всякое семейство $\left(u_{1}, \ldots, u_{n+1}\right)$ m-покрытий пространства $X$ несущественно;

3) всякое семейство $\left(u_{1}, \Phi_{1}\right), \ldots,\left(u_{n+1}, \Phi_{n+1}\right)$ m-накрывающих пар пространства $X$ несущественно;

4) всякое семейство $\left(\Phi_{1}, \ldots, \Phi_{n+1}\right)$ m-систем пространства $X$ несущественно.

ДокАЗАТЕЛЬство. Пусть $\operatorname{dim} X \leqslant n$. В покрытие $u_{1} \wedge \cdots \wedge u_{n+1}$ по теореме Остранда можно вписать покрытие, распадающееся в объединение дизъюнктных семейств $v_{1}, \ldots, v_{n+1}$. При этом каждое из семейств $v_{i}$ вписано в любое покрытие $u_{j}$, в частности, в $u_{i}$. Тем самым свойство 2) выполнено.

Проверим импликацию 2) $\Rightarrow 3$ ). Пусть $u_{i}=\left\{U_{1}^{i}, \ldots, U_{k_{i}}^{i}\right\}, \Phi_{i}=\left\{F_{1}^{i}, \ldots, F_{k_{i}}^{i}\right\}$. Существуют такие окрестности $O_{1} F_{j}^{i}$, что каждое семейство

$$
\left\{\left[O_{1} F_{j}^{i}\right]: j=1, \ldots, k_{i}\right\}
$$

дизъюнктно и вписано в $u_{i}$. Полагаем

$$
{ }^{1} U_{j}^{i}=U_{j}^{i} \backslash \bigcup\left\{\left[O_{1} F_{l}^{i}\right]: l \neq j\right\} \quad \text { и } \quad u_{i}^{1}=\left\{{ }^{1} U_{1}^{i}, \ldots,{ }^{1} U_{k_{i}}^{i}\right\} .
$$

Каждое семейство $u_{i}^{1}$ является покрытием $X$. Согласно 2), существует последовательность $v_{i}=\left\{V_{1}^{i}, \ldots, V_{k_{i}}^{i}\right\}$ дизъюнктных открытых семейств, комбинаторно 
$C$-вписанная в последовательность $\left(u_{i}\right)$. Полагая

$$
O F_{j}^{i}=V_{j}^{i} \cup O_{1} F_{j}^{i} \quad \text { и } \quad O \Phi_{i}=\left\{O F_{1}^{i}, \ldots, O F_{k_{i}}^{i}\right\}
$$

получаем окрестности систем $\Phi_{i}$, реализующие несущественность семейства $\left(u_{1}, \Phi_{1}\right), \ldots,\left(u_{n+1}, \Phi_{n+1}\right)$.

Свойство 4) является следствием свойства 3$)$, примененного к парам $\left(u_{i}, \Phi_{i}\right)$, где $u_{i}=\left\{U_{1}^{i}, \ldots, U_{k_{i}}^{i}\right\}$ и $U_{j}^{i}=X \backslash \bigcup\left\{F_{l}^{i}: l \neq j\right\}$. Наконец, из $\left.4_{m}\right)$ следует свойство $4_{2}$ ), равносильное теореме о перегородках. Теорема 4.1 доказана.

Теперь мы модифицируем некоторые определения и факты, содержащиеся в работах Р. Поля [47] и П. Борста [48].

ОПРЕДЕЛЕниЕ 4.2. Через Fin $\mathbb{N}$ обозначим множество всех непустых конечных подмножеств $\mathbb{N}$, снабженное следующим порядком $\prec: ~ \sigma \prec \tau$ означает существование такого $n \in \sigma \backslash \tau$, что

$$
\sigma \cap\{1, \ldots, n-1\}=\tau \cap\{1, \ldots, n-1\}
$$

Этот порядок называется порядком Брауэра-Клини.

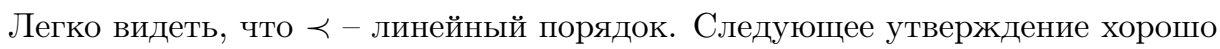
известно (см., например, [47; лемма 1.1]).

ЛЕмма 4.3. Для убъвающей последовательности $\sigma_{1} \succ \sigma_{2} \succ \cdots$ элементов Fin $\mathbb{N}$ существует такая возрастающая последовательность $j(1)<j(2)<\cdots$ натуральных чисел, что для каждого $k$ существует l со свойством $\{j(1), \ldots$, $j(k)\} \subset \sigma_{l}$.

Последовательность $R=\left\{u_{i}: i \in \mathbb{N}\right\}$ попарно различных $m$-покрытий компакта $X$ называется $m$-вписъваемой, если для любого существенного покрытия $u \in \operatorname{cov}_{m}(X)$ бесконечно множество

$$
\left\{i \in \mathbb{N}: u_{i} \succ u\right\}
$$

где отношение $v \succ w$ означает, что $m$-покрытие $v$ комбинаторно вписано в $m$-покрытие $w$. Ясно, что все элементы $m$-вписываемой последовательности существенны.

Лемма 4.4. Для произвольного конечного семейства существенных $m$-покрытий $u_{1}, \ldots, u_{k}$, и пространства $X$ существует $m$-покрытие $u_{k+1}$, вписанное в и и отличающееся от всех $u_{1}, \ldots, u_{k}$.

ДоКАЗАТЕЛЬСТво. Если ни одно из покрытий $u_{j}$ не вписано в $u$, то полагаем $u_{k+1}=u$. В противном случае среди покрытий $u_{j} \succ u$ берем максимальное $u_{j_{0}}$. В это покрытие комбинаторно с замыканием вписываем покрытие, которое и обозначаем $u_{k+1}$. Тогда $u_{k+1} \neq u_{j_{0}}$, поскольку в противном случае все элементы покрытия $u_{j_{0}}$ открыто-замкнуты, что противоречит его существенности.

ПреДЛОЖЕНИЕ 4.5. Всякий метризуемый компакт обладает $m$-вписываемой последовательностъю $R$. 
ДокаЗАТЕЛЬСтво. В множестве $\operatorname{cov}_{m} X$ выберем счетное конфинальное подмножество $\mathscr{V}$. Обозначим через $\mathscr{V}_{0}$ множество всех существенных $v \in \mathscr{V}$. Занумеруем элементы $\mathscr{V}_{0}$ натуральными числами так, что каждый элемент $v \in \mathscr{V}_{0}$ приобретает бесконечное число номеров: $\mathscr{V}_{0}=\left\{v_{i}: i \in \mathbb{N}\right\}$. Теперь строим последовательность $R=\left\{u_{i}: i \in \mathbb{N}\right\}$ по рекурсии. Полагаем $u_{1}=v_{1}$. Предполагая, что покрытия $u_{1}, \ldots, u_{k}$ построены, применяем к последовательности $u_{1}, \ldots, u_{k}, u$, где $u=v_{k+1}$, лемму 4.4. Предложение 4.5 доказано.

Для произвольной $m$-вписываемой последовательности $R$ компакта $X$ положим

$$
M_{R}(X)=\left\{\sigma \in \operatorname{Fin} \mathbb{N}: \text { семейство }\left\{u_{i}: i \in \sigma, u_{i} \in R\right\} \text { существенно }\right\} \text {. }
$$

Будем рассматривать множество $M_{R}(X)$ с порядком Брауэра-Клини.

Лемма 4.6. Пусть $R_{j}=\left\{u_{i}^{j}: i \in \mathbb{N}\right\}, j=1,2,-m$-вписываемые последовательности компакта $X$. Тогда упорядоченное множество $M_{R_{1}}(X)$ подобно подмножеству из $M_{R_{2}}(X)$ и наоборот.

ДокАЗАТЕЛьство. Пользуясь бесконечностью множества (4.1), выберем такую последовательность $j(1)<j(2)<\cdots$, что $u_{j(i)}^{2} \succ u_{i}^{1}$. Если $\sigma \in M_{R_{1}}(X)$, то $j(\sigma) \in M_{R_{2}}(X)$. Отображение $\sigma \rightarrow j(\sigma)$ и осуществляет сохраняющее порядок вложение $M_{R_{1}}(X) \rightarrow M_{R_{2}}(X)$.

Лемму 4.6 для $m=2$ и $m=\infty$ доказали соответственно Р. Поль [47] и В. А. Чатырко [49].

Теорема 4.7. Компакт $X$ является $m$-C-пространством тогда и только тогда, когда для некоторой (эквивалентно - для каждой) т-вписываемой последовательности $R$ компакта $X$ множество $M_{R}(X)$ вполне упорядочено.

ДоказАтельство. Пусть $R=\left\{u_{i}: i \in \mathbb{N}\right\}-m$-вписываемая последовательность. Если $X \notin m$ - $C$, то существует бесконечная существенная последовательность $\left\{v_{i}: i \in \mathbb{N}\right\} \quad m$-покрытий $X$. Существует такая последовательность $j(1)<j(2)<\cdots$, что $u_{j(i)} \succ v_{i}$. Тогда $\sigma_{i}=\{j(1), \ldots, j(i)\} \in M_{R}(X)$ и $\sigma_{1} \succ \sigma_{2} \succ \cdots$. Следовательно, множество $M_{R}(X)$ не вполне упорядочено.

Наоборот, предположим, что существуют $\sigma_{i} \in M_{R}(X)$, для которых $\sigma_{1} \succ$ $\sigma_{2} \succ \cdots$. Возьмем последовательность $j(1)<j(2)<\cdots$ из леммы 4.3. Тогда бесконечное семейство $\mathscr{U}=\left\{u_{j(i)}: i \in \mathbb{N}\right\}$ существенно. В самом деле, для любого $k \in \mathbb{N}$ существует такое $l \in \mathbb{N}$, что $\{j(1), \ldots, j(k)\} \subset \sigma_{l}$. Следовательно, все начальные отрезки $\left\{u_{j(1)}, \ldots, u_{j(k)}\right\}$ семейства $\mathscr{U}$ существенны. Отсюда, в силу компактности $X$, вытекает существенность $\mathscr{U}$.

Для $m=2$ теорему 4.7 доказал Р. Поль [47], для $m=\infty$ - В. А. Чатырко [49].

ОПРЕДЕЛЕНИЕ 4.8 . Для $m$ - $C$-компакта $X$ положим

$$
\operatorname{index}_{m} X=\text { type } M_{R}(X)
$$

где $R$ - некоторая (любая) $m$-вписываемая последовательность в $X$. 
Из счетности множества $R$ и теоремы 4.7 вытекает, что index $m X$ - счетное порядковое число, называемое $m$-индексом Лузина-Серпинского. Функции

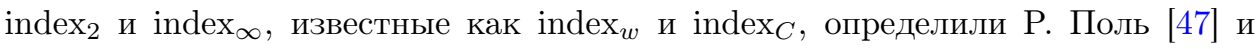
В. А. Чатырко [49] соответственно.

ПреДЛОЖЕНИЕ 4.9. Если $m_{1} \leqslant m_{2}$, mo index $m_{m_{1}} X \leqslant \operatorname{index}_{m_{2}} X$.

ДоказАтельство. Пусть $R=\left\{u_{i}: i \in \mathbb{N}\right\}-m_{2}$-вписываемая последовательность в компакте $X$. Положим $R_{1}=\left\{u_{i} \in R:\left|u_{i}\right| \leqslant m_{1}\right\}$ и $N_{1}=\{i \in \mathbb{N}$ : $\left.\left|u_{i}\right| \leqslant m_{1}\right\}$. Тогда $R_{1}-m_{1}$-вписываемая последовательность в $X$, а тождественое вложение $N_{1} \rightarrow \mathbb{N}$ порождает вложение множества $M_{R_{1}}(X)$ в $M_{R}(X)$. Следовательно, type $M_{R_{1}}(X) \leqslant$ type $M_{R}(X)$, что и доказывает предложение 4.9 .

ПреДЛОЖЕНИЕ 4.10. Если $F$ - замкнутое подмножество $m$-C-компакma $X$, mo

$$
\operatorname{index}_{m} F \leqslant \operatorname{index}_{m} X .
$$

ДокаЗАтельство. Пусть $u=\left\{U_{1}, \ldots, U_{k}\right\}$ - открытое покрытие компакта $F$. Полагая $e(u)=\left\{e\left(U_{1}\right), \ldots, e\left(U_{k}\right)\right\}$, где $e\left(U_{j}\right)=U_{j} \cup(X \backslash F)$, получаем открытое покрытие компакта $X$. Получаем оператор $e: \operatorname{cov}_{m}(F) \rightarrow \operatorname{cov}_{m}(X)$ вложения. Этот оператор переводит существенные последовательности в существенные. Возьмем множество $N_{1} \subset \mathbb{N}$ так, что и $N_{1}$, и $\mathbb{N} \backslash N_{1}$ бесконечны. Занумеруем элементами из $N_{1}$ какую-нибудь $m$-вписываемую последовательность $R=\left\{u_{i}: i \in N_{1}\right\}$ компакта $F$. Семейство $e(R)=\left\{e\left(u_{i}\right): i \in N_{1}\right\}$ дополним до $m$-вписываемой последовательности $R_{1}=\left\{v_{i}: i \in \mathbb{N}\right\}$ компакта $X$. Для этого достаточно взять существующую согласно предложению $4.5 \mathrm{~m}$-вписываемую последовательность $R_{2}=\left\{v_{i}: i \in \mathbb{N} \backslash N_{1}\right\}$ и положить $R_{1}=e(R) \cup R_{2}$. Тождественное вложение $N_{1} \subset \mathbb{N}$ порождает сохраняющее порядок $\prec$ вложение множества $M_{R}(F)$ в $M_{R_{1}}(X)$. Предложение 4.10 доказано.

Фиксируем $m$-вписываемую последовательность $R=\left\{u_{i}: i \in \mathbb{N}\right\}$ в гильбертовом кубе $Q=I^{\omega}$. Для $\sigma \in \operatorname{Fin} \mathbb{N}$ положим

$$
\bar{W}_{\sigma}=\left\{X \in \exp Q: \text { семейство }\left\{u_{i} \mid X: i \in \sigma\right\} \text { существенно }\right\} .
$$

УТВЕРЖДЕНИЕ 4.11. Каждое множество $\bar{W}_{\sigma}$ замкнуто в $\exp Q$.

ДоказАтельство. Пусть $X \notin \bar{W}_{\sigma}$ и $u_{i}=\left\{U_{1}^{i}, \ldots, U_{k_{i}}^{i}\right\}$. Существуют такие дизъюнктные открытые в $Q$ семейства $v_{i}=\left\{V_{1}^{i}, \ldots, V_{k_{i}}^{i}\right\}, i \in \sigma$, что $V_{j}^{i} \subset U_{j}^{i}$ и $X \subset \bigcup\left\{V_{j}^{i}: i \in \sigma, j \leqslant k_{i}\right\}$. Тогда множество

$$
O\left\langle V_{j}^{i}: i \in \sigma, j \leqslant k_{i}, V_{j}^{i} \cap X \neq \varnothing\right\rangle
$$

будет окрестностью компакта $X$ в $\exp Q$, не пересекающейся с $\bar{W}_{\sigma}$.

Таким образом, отождествляя множества $\sigma=\left\{i_{1}, \ldots, i_{n}\right\} \in \operatorname{Fin} \mathbb{N}$ с двоичнорациональными дробями

$$
r=\frac{1}{2^{i_{1}}}+\cdots+\frac{1}{2^{i_{n}}}
$$

получаем, что семейство

$$
\bar{W}=\left\{\bar{W}_{\sigma}: \sigma \in \operatorname{Fin} \mathbb{N}, \prec\right\}
$$


является решетом Лузина (см. [50; §3, XV]), состоящим из замкнутых множеств компакта $\exp Q$.

Для компакта $X \in \exp Q$ положим

$$
M(X)=\left\{\sigma \in \operatorname{Fin} \mathbb{N}: X \in \bar{W}_{\sigma}\right\} .
$$

Напомним [50; $3, \mathrm{XV}]$, что множество

$$
A=A(\bar{W})=\{X \in \exp Q:\{M(X) \text { не вполне упорядочено }\}\}
$$

является просеянным через решето $\bar{W}$.

Из утверждения 4.11, теоремы 4.7 и вышесказанного вытекает

$$
A(\bar{W})=\{X: X \notin m-C\} .
$$

Следовательно, дополнение

$$
-A \equiv(\exp Q) \backslash A
$$

состоит из $m$ - $C$-компактов и, значит,

$$
-A=\bigcup\left\{A_{\alpha}: \alpha<\omega_{1}\right\}
$$

где

$$
A_{\alpha}=\left\{X: \operatorname{index}_{m} X=\alpha\right\} .
$$

Tеорема A [50; §39, VIII, теорема 3]. Для каждого $\alpha<\omega_{1}$ множество $A_{\alpha}$ является борелевским.

Теорема В [50; § 39, VIII, следствие 5a]. Для каждого аналитического множества, не пересекающегося с множеством, просеянным через решето, индексы Лузина-Серпинского его точек ограничены счетным порядковым числом.

Лемма 4.12 (см. [47; лемма 5.2]). Пусть $Y \subset Q-$ компакт. Тогда множество

$$
E_{Y}=\{X \in \exp Q: X \text { вкладьвается в } Y\}
$$

является аналитическим.

В самом деле, $E_{Y}$ является проекцией замкнутого в $\exp Q \times C(Q, Q)$ множества

$$
\{(X, f): f(X) \subset Y, f \mid X-\text { вложение }\} .
$$

ПрЕДЛОЖЕНИЕ 4.13 (см. [47; лемма 5.2]). Пусть $Z \subset \exp Q-$ некоторое множество. Для того чтобы существовал т-C-компакт $Y$, топологически содержащий все $X \in Z$, необходимо и достаточно, чтобъ существовало аналитическое множество $E \subset \exp Q$, содержащее $Z$ и состоящее из $m$ - $C$ компактов. 
ДокАЗАТЕЛЬство. Пусть такой компакт $Y$ существует. Тогда множество $E_{Y}$ по лемме 4.12 является аналитическим и согласно предложению 4.10 содержит все $X \in Z$ и состоит из $m$ - $C$-компактов.

Наоборот, предположим, что такое аналитическое множество $E$ существует. Пусть $\varphi: \mathbb{N}^{\mathbb{N}} \rightarrow E-$ непрерывная сюръекция. Положим $G=\{(t, x): x \in \varphi(t)\}$. Проектирование $\pi: G \rightarrow \mathbb{N}^{\mathbb{N}}$ является совершенным отображением с $m$ - $C$-слоями $\pi^{-1}(t)$. Тогда $G$ является $m$ - $C$-пространством согласно теореме 2.2 (теореме 5.2 из [15]). Далее, $G$ топологически полно как замкнутое подмножество $\mathbb{N}^{\mathbb{N}} \times Q$. По теореме 3.66 из [15] пространство $G$ имеет расширение $Y$, являющееся $m$ - $C$-компактом. Предложение 4.13 доказано.

Из теоремы А и предложений 4.10 и 4.13 вытекает следующая теорема.

Теорема 4.14 (см. [47; теорема 5.1]). Для того чтобъ существовал $m$ - $C$ компакт, топологически содержащий все $m$-C-компакты данного семейства Е, необходимо и достаточно, чтобъ

$$
\sup \left\{\operatorname{index}_{m} X: X \in E\right\}<\omega_{1} .
$$

ОПРЕДЕЛЕНИЕ 4.15. Пусть $L-$ произвольное множество, и пусть $M \subset$ Fin $L$. Для $\sigma \in\{\varnothing\} \cup \operatorname{Fin} L$ положим

$$
M^{\sigma}=\{\tau \in \operatorname{Fin} L: \sigma \cup \tau \in M, \sigma \cap \tau=\varnothing\} .
$$

Для $a \in L$ множество $M^{\{a\}}$ будем обозначать $M^{a}$.

Если $\sigma, \tau \in \operatorname{Fin} L$ и $\sigma \cap \tau=\varnothing$, то

$$
\begin{gathered}
\left(M^{\sigma}\right)^{\tau}=M^{\sigma \cup \tau} ; \\
\sigma \in M^{\tau} \Longleftrightarrow \tau \in M^{\sigma} .
\end{gathered}
$$

Порядковое число Ord $M$ определяется по индукции.

1) $\operatorname{Ord} M=0 \Longleftrightarrow M=\varnothing$.

2) $\operatorname{Ord} M \leqslant \alpha \Longleftrightarrow \operatorname{Ord} M^{a}<\alpha$ для любого $a \in L$.

3) Ord $M=\alpha \Longleftrightarrow$ Ord $M \leqslant \alpha$ и Ord $M<\alpha$ не выполняется.

4) $\operatorname{Ord} M=\infty$ (не существует) $\Longleftrightarrow \operatorname{Ord} M>\alpha$ для всякого порядкового числа $\alpha$.

Ясно, что

$$
N \subset M \Longrightarrow \operatorname{Ord} N \leqslant \operatorname{Ord} M .
$$

Множество $M \subset$ Fin $L$ называется включающим, если для любых $\sigma_{1}, \sigma_{2} \in$ Fin $L$ из $\sigma_{1} \in M$ и $\sigma_{2} \subset \sigma_{1}$ вытекает $\sigma_{2} \in M$.

Лемма 4.16 [48]. Если $M \subset$ Fin $L$ - включающее множество, $\operatorname{mo}$ Ord $M=$ $\infty$ тогда и только тогда существует такая последовательность $\left\{a_{i}: i \in \mathbb{N}\right\}$ различных элементов из $L$, что

$$
\sigma_{n} \equiv\left\{a_{1}, \ldots, a_{n}\right\} \in M
$$

для каждого $n \in \mathbb{N}$.

Лемма 4.17 [48]. Если $M \subset$ Fin $L$ u $n \in \mathbb{N}$, mo Ord $M \leqslant n$ тогда и только тогда, когда $|\sigma| \leqslant n$ для всякого $\sigma \in M$. 
Лемма 4.18 [48]. Пусть $\mu: L \rightarrow L_{1}$ - отображение, $M \subset \operatorname{Fin} L, M_{1} \subset$ Fin $L_{1}$. Предположим также, что $\mu(\sigma) \in M_{1} u|\mu(\sigma)|=|\sigma|$ для всякого $\sigma \in M$. Тогда $\operatorname{Ord} M \leqslant \operatorname{Ord} M_{1}$.

Положим

$$
M_{m}(X)=\left\{\sigma \in \text { Fin } \operatorname{cov}_{m}(X): \sigma \text { существенно }\right\} .
$$

Следующее утверждение, вытекающее из теоремы 4.1 и леммы 4.17, является обобщением теорем Борста [48], [51] для $m=2, \infty$ соответственно.

Теорема 4.19. Для нормалъного пространства $X$ имеем

$$
\operatorname{dim} X \leqslant n \Longleftrightarrow \operatorname{Ord} M_{m}(X) \leqslant n .
$$

ОПРЕДЕлЕНиЕ 4.20. Для нормального пространства $X$ полагаем

$$
\operatorname{dim}_{m} X=\operatorname{Ord} M_{m}(X) .
$$

В случае $\operatorname{dim}_{m} X=\infty$ будем говорить, что размерность $\operatorname{dim}_{m} X$ не определена.

Согласно теореме 4.19 инвариант $\operatorname{dim}_{m}$ является трансфинитным продолжением лебеговой размерности на бесконечномерные пространства.

Из леммы 4.16 вытекает следующая теорема.

Теорема 4.21. Размерность $\operatorname{dim}_{m} X$ определена тогда и толъко тогда, когда $X$ есть $S$-m-C-пространство.

Для $m=2, \infty$ это утверждение доказал Борст [48], [51].

Следствием леммы 4.18 является следующее утверждение.

ПРЕДЛОЖЕНИЕ 4.22. Если $m_{1} \leqslant m_{2}, m o$

$$
\operatorname{dim}_{m_{1}} X \leqslant \operatorname{dim}_{m_{2}} X .
$$

Снова применяя лемму 4.18 и оператор $e: \operatorname{cov}_{m}(F) \rightarrow \operatorname{cov}_{m}(X)$ из доказательства предложения 4.10, получаем

ПреДЛОЖЕНИЕ 4.23. Если $F \subset X$ - замкнутое подмножество, то

$$
\operatorname{dim}_{m} F \leqslant \operatorname{dim}_{m} X
$$

Пусть теперь снова $L$ - непустое множество и $M \subset \operatorname{Fin} L$. Для $a \in L$ положим

$$
M[a]=\{\tau \in M: a \in \tau\}, \quad M[\bar{a}]=\{\tau \in M: a \notin \tau\} .
$$

Ясно, что $M=M[a] \sqcup M[\bar{a}]$.

ОПРЕДЕЛЕНИЕ 4.24 [49]. Назовем непустое множество $M \subset$ Fin $\mathbb{N}$ широким, если:

(a) $M-$ включающее;

(b) для всякого $a \in \mathbb{N} \cap M$ найдется бесконечно много таких различных $a_{1} \in \mathbb{N} \cap M$, что

$\left(\mathrm{b}_{1}\right) a_{1} \neq a$;

$\left(\mathrm{b}_{2}\right) M^{a}\left[\bar{a}_{1}\right] \subset M\left[a_{1}\right]$. 
Лемма 4.25 [48]. Для включающего множества $M \subset$ Fin $\mathbb{N}$ условие $\operatorname{Ord} M \neq \infty$ равносильно тому, что $M$ вполне упорядочено порядком Брауэра-Клини.

TеOрема 4.26 [49]. Если Ord $M \neq \infty$ для некоторого широкого множества $M \subset \operatorname{Fin} \mathbb{N}, m o$

$$
\text { type } M=\omega^{\text {Ord } M} \text {. }
$$

Лемма 4.27 [49]. Пусть $L_{1} \subset L_{2}, M_{i} \subset$ Fin $L_{i}-$ такие непустые включающие множества, что $M_{1} \subset M_{2}$. Пусть также выполнены следующие условия:

1) для любого $a_{2} \in L_{2} \cap M_{2}$ найдется бесконечно много таких различных элементов $a_{1} \in L_{1} \cap M_{1}$, что $a_{1} \neq a_{2} u M_{2}^{a_{2}}\left[\bar{a}_{1}\right] \subset M_{2}^{a_{1}}$;

2) для любого $\sigma \in M_{1}$ условия $M_{2}^{\sigma} \neq \varnothing$ и $M_{1}^{\sigma} \neq \varnothing$ равносильны;

3) для любых различных элементов $a_{1}, \ldots, a_{k} \in L_{1}$ если $\sigma=\left\{a_{1}, \ldots, a_{k}\right\} \in$ $M_{2}, \operatorname{mo} \sigma \in M_{1}$.

Тогда $\operatorname{Ord} M_{1}=\operatorname{Ord} M_{2}$.

Для $L \subset \operatorname{cov}_{m}(X)$ положим

$$
M_{L}=\{\sigma \in \operatorname{Fin} L: \sigma \text { существенно }\} .
$$

Следующее утверждение для $m=\infty$ доказано в [49; лемма 10].

ПРЕДЛОЖЕНИЕ 4.28. Пусть $X-m$-C-компакт, $\operatorname{dim} X \neq 0 u R=\left\{u_{i}:\right.$ $i \in \mathbb{N}\}-m$-вписываемая последовательность. Тогда

(a) $\operatorname{Ord} M_{R}(X)=\operatorname{Ord} M_{R}=\operatorname{Ord} M_{m}(X)$;

(b) множество $M_{R}(X) \subset$ Fin $\mathbb{N}$ широкое и вполне упорядочено порядком Брауэра-Клини.

Доказательство. (а) Равенство $\operatorname{Ord} M_{R}(X)=\operatorname{Ord} M_{R}$ вытекает из леммы 4.18 после естественного отождествления последовательности $R=\left\{u_{i}\right.$ : $i \in \mathbb{N}\}$ и множества $\mathbb{N}$.

Теперь положим $L_{1}=R, L_{2}=\operatorname{cov}_{m} X, M_{1}=M_{R}$ и $M_{2}=M_{m}(X)$. Множество $M_{1}$ непусто, так как $\operatorname{dim} X \neq 0$. Далее, $M_{1}, M_{2}$ - включающие множества и $M_{1} \subset M_{2}$. Поэтому для доказательства равенства $\operatorname{Ord} M_{R}=\operatorname{Ord} M_{m}(X)$ достаточно проверить выполнение свойств 1)-3) из леммы 4.27.

1) По определению $m$-вписываемой последовательности для любого $u \in$ $M_{m}(X) \cap \operatorname{cov}_{m} X$ существует бесконечно много таких чисел $i$, что $u_{i} \succ u, u_{i} \neq u$ и все $u_{i}$ попарно различны. Покажем, что $M_{2}^{u}\left[\bar{u}_{i}\right] \subset M_{2}^{u_{i}}$. Пусть $\tau \in M_{2}^{u}\left[\bar{u}_{i}\right]$. Это означает, что $\tau \in M_{2}^{u}$ и $u_{i} \notin \tau$. Из $\tau \in M_{2}^{u}$ вытекает существенность семейства $\tau \cup u$, что, в силу вписанности $u_{i}$ в $u$, влечет существенность $\tau \cup u_{i}$. Следовательно, $\tau \in M_{2}^{u_{i}}$.

2) Пусть $\sigma \in M_{1}$ и $M_{2}^{\sigma} \neq \varnothing$, т.е. найдется $\tau \in M_{2}^{\sigma}$. Пользуясь $m$-вписываемостью последовательности $R$, можно, поэлементно вписывая покрытия в элементы из $\tau$, подобрать такое $\tau_{1} \in M_{1}$, что $\tau_{1} \cap \sigma=\varnothing$. Из существенности $\tau \cup \sigma\left(\tau \in M_{2}^{\sigma}\right)$ вытекает существенность более мелкого семейства $\tau_{1} \cup \sigma$. Следовательно, $\tau_{1} \in M_{1}^{\sigma}$.

3) Очевидно.

Итак, $\operatorname{Ord} M_{R}=\operatorname{Ord} M_{m}(X)$. 
(b) По теореме 4.21 имеем $\operatorname{Ord} M_{m}(X) \neq \infty$. Следовательно, из уже доказанного пункта (a) получаем $\operatorname{Ord} M_{R}(X) \neq \infty$. Поэтому согласно лемме 4.25 множество $M_{R}(X)$ вполне упорядочено порядком Брауэра-Клини. Наконец, из $m$-вписываемости последовательности $R$ вытекает, что множество $M_{R}(X) \equiv M$ удовлетворяет определению 4.24. Предложение 4.28 доказано.

Из предложений 4.5 и 4.28, теорем 4.21 и 4.26 и определений 4.8 и 4.20 вытекает следующая теорема.

Tеорема 4.29. Если $X-m$-C-компакт $u \operatorname{dim} X \neq 0, m o$

$$
\operatorname{index}_{m} X=\omega^{\operatorname{dim}_{m} X} .
$$

Для $m=2$ эту теорему доказал П. Борст [49], а для $m=\infty-$ В. А. Чатырко [49].

Следствием теорем 4.14 и 4.29 является

ТеОремА 4.30. Для того чтобы существовал m-C-компакт, топологически содержащий все m-C-компакты данного семейства $E$, необходимо и достаточно, чтобъ

$$
\sup \left\{\operatorname{dim}_{m} X: X \in E\right\}<\omega_{1} .
$$

ЗАмечание 4.31. Трансфинитную классификацию допускают и $w-m-C$ компакты. Разумеется, каждый $w-m$ - $C$-компакт $X$ слабо бесконечномерен и для него определены размерностные функции $\operatorname{index}_{2} X$ и $\operatorname{dim}_{2} X$. Но можно ввести функции index $_{w m}$ и $\operatorname{dim}_{w m}$ так, что для компакта $X$ они определены тогда и только тогда, когда $X \in w-m-C$. Надо повторить все предыдущие рассуждения, заменив $m$-покрытия на $m$-системы (см. определение 1.11 ). Ситуация здесь несколько проще, поскольку, как и в случае дизъюнктных пар замкнутых множеств, всякое семейство $\left\{\Phi_{i}\right\} m$-систем, содержащее две одинаковые системы, несущественно. Приведем основные результаты.

ОПРЕДЕЛЕНИЕ 4.32. Последовательность $S=\left\{\Phi_{i}: i \in \mathbb{N}\right\} \quad m$-систем компакта $X$ называется $M$-разделяющей, если для всякой $m$-системы $\Phi$ бесконечно множество $\left\{i \in \mathbb{N}: \Phi \succ \Phi_{i}\right\}$.

Отметим, что здесь мы не требуем попарной различности систем $\Phi_{i}$. Поэтому $m$-разделяемость $S$ равносильна тому, что семейство $S$ конфинально в множестве $\varphi_{m}(X)$ всех $m$-систем, упорядоченном отношением, обратным отношению вписанности. У нас нет необходимости доказывать аналог леммы 4.6, хотя, разумеется, он имеет место.

Для произвольной $m$-разделяющей последовательности $S=\left\{\Phi_{i}: i \in \mathbb{N}\right\}$ компакта $X$ полагаем

$$
M_{S}(X)=\left\{\sigma \in \operatorname{Fin} \mathbb{N}: \text { семейство }\left\{\Phi_{i}: i \in \sigma, \Phi_{i} \in S\right\} \text { существенно }\right\} .
$$

Теорема 4.33. Компакт $X$ является $w$-m-C-пространством тогда $и$ только тогда, когда для некоторой (эквивалентно - для всякой) т-разделяющей последовательности $S$ в $X$ множество $M_{S}(X)$ вполне упорядочено порядком Брауэра-Клини. 
ОПРЕДЕЛЕНИЕ 4.34 . Для $w-m$ - $C$-компакта $X$ положим

$$
\operatorname{index}_{w m} X=\text { type } M_{s}(X),
$$

где $S$ - некоторая (всякая) $m$-разделяющая последовательность в $X$.

ПреДЛОЖение 4.35. Если $m_{1} \leqslant m_{2}, m o \operatorname{index}_{w m_{1}} X \leqslant \operatorname{index}_{w m_{2}} X$.

ПреДЛОЖЕНИЕ 4.36. Если $F$ - замкнутое подмножество $m$-C-компакma $X$, mo

$$
\operatorname{index}_{w m} F \leqslant \operatorname{index}_{w m} X \text {. }
$$

ПреДЛОЖЕНИЕ 4.37. Пусть $Z \subset \exp Q$ - некоторое множество. Для того чтобъ существовал w-m-C-компакт $Y$, топологически содержащий все $X \in Z$, необходимо и достаточно, чтобы существовало аналитическое множество $E \subset \exp Q$, содержащее $Z$ и состоящее из $w-m-C$-компактов.

Теорема 4.38. Для того чтобы существовал w-m-C-компакт, топологически содержащий все $w$-т-C-компакты данного семейства $E$, необходимо и достаточно, чтобъ

$$
\sup \left\{\operatorname{index}_{w m} X: X \in E\right\}<\omega_{1} .
$$

Положим

$$
M_{w m}(X)=\left\{\sigma \in \operatorname{Fin} \varphi_{m}(X): \sigma \text { существенно }\right\} .
$$

Теорема 4.39. Для нормалъного пространства $X$ имеем

$$
\operatorname{dim} X \leqslant n \Longleftrightarrow \operatorname{Ord} M_{w m}(X) \leqslant n .
$$

ОПРЕДЕЛЕНИЕ 4.40. Для нормального пространства $X$ полагаем

$$
\operatorname{dim}_{w m} X=\operatorname{Ord} M_{w m}(X) .
$$

В случае $\operatorname{dim}_{w m} X=\infty$ будем говорить, что размерность $\operatorname{dim}_{w m} X$ не определена.

ТеОрема 4.41. Размерность $\operatorname{dim}_{w m} X$ пространства $X$ определена тогда и только тогда, когда $X$ есть $S$-w-m-C-пространство.

ПРЕДЛОЖЕНИЕ 4.42. Если $m_{1} \leqslant m_{2}, m_{o}$

$$
\operatorname{dim}_{w m_{1}} X \leqslant \operatorname{dim}_{w m_{2}} X .
$$

ПреДЛОЖЕНИЕ 4.43. Если $F \subset X-$ замкнутое подмножество, то

$$
\operatorname{dim}_{w m} F \leqslant \operatorname{dim}_{w m} X .
$$

Пусть $\Phi=\left\{F_{1}, \ldots, F_{k}\right\}-m$-система пространства $X$. Определим покрытие $\mu(\Phi)=\left\{U_{1}, \ldots, U_{k}\right\}$, полагая

$$
U_{i}=X \backslash \bigcup\left\{F_{j}: j \neq i\right\}
$$

Получаем отображение $\mu: \varphi_{m}(X) \rightarrow \operatorname{cov}_{m}(X)$. Отметим, что покрытия $\mu(\Phi)$ являются частным случаем (для конечных $\Phi)$ покрытий с $(\Phi)$ из $(2.1)$. 
Лемма 4.44. Если конечное семейство т-систем $\sigma=\left\{\Phi_{1}, \ldots, \Phi_{s}\right\}$ существенно, то семейство покрытий $\mu(\sigma)=\left\{\mu\left(\Phi_{1}\right), \ldots, \mu\left(\Phi_{s}\right)\right\}$ существенно.

ДокАЗАтельство. Пусть $\Phi_{l}=\left\{F_{1}^{l}, \ldots, F_{k_{l}}^{l}\right\}$ и $\mu\left(\Phi_{l}\right)=\left\{U_{1}^{l}, \ldots, U_{k_{l}}^{l}\right\}$. Предположим, что $\mu(\sigma)$ несущественно. Тогда существуют такие дизъюнктные открытые семейства

$$
v_{l}=\left\{V_{1}^{l}, \ldots, V_{k_{l}}^{l}\right\}, \quad 1 \leqslant l \leqslant s,
$$

что

$$
\left[V_{j}^{l}\right] \subset U_{j}^{l}
$$

и

$$
\bigcup\left\{V_{j}^{l}: 1 \leqslant l \leqslant s, 1 \leqslant j \leqslant k_{l}\right\}=X .
$$

Из (4.6) и (4.8) вытекает, что

$$
\left[V_{j_{1}}^{l}\right] \cap F_{j_{2}}^{l}=\varnothing \quad \text { при } \quad j_{1} \neq j_{2} .
$$

Следовательно, существуют такие окрестности $O_{1} \Phi_{l}=\left\{O_{1} F_{1}^{l}, \ldots, O_{1} F_{k_{l}}^{l}\right\}$, что

$$
O_{1} F_{j_{1}}^{l} \cap V_{j_{2}}^{l}=\varnothing \quad \text { при } \quad j_{1} \neq j_{2} .
$$

Поэтому, полагая $O F_{j}^{l}=O_{1} F_{j}^{l} \cup V_{j}^{l}$, получаем окрестности $O \Phi_{l}=\left\{O F_{1}^{l}, \ldots\right.$, $\left.O F_{k_{l}}^{l}\right\}$ систем $\Phi_{l}$, тела которых согласно (4.8) покрывают $X$. Получили противоречие с существенностью семейства $\sigma$. Лемма 4.44 доказана.

Таким образом, отображение $\mu$ удовлетворяет условиям леммы 4.18 , согласно которой

$$
\operatorname{Ord} M_{w m}(X) \leqslant \operatorname{Ord} M_{m}(X) .
$$

Итак, нами доказана следующая теорема.

ТеОРема 4.45. Для любого нормального пространства $X$ и всякого $m$ имеeм $\operatorname{dim}_{w m} X \leqslant \operatorname{dim}_{m} X$.

В отличие от размерности $\operatorname{dim}_{m}$ можно доказать аналог теоремы Борста [48; теорема 3.2.4] для $\operatorname{dim}_{w m}$. А именно, имеет место следующая теорема.

ТеоремА 4.46. Если трансфинитная размерность Ind $X$ определена, то

$$
\operatorname{dim}_{w m} X \leqslant \operatorname{Ind} X
$$

Теорема 4.47. Если $X-w-m-C$-компакт $u \operatorname{dim} X \neq 0$, mо

$$
\operatorname{index}_{w m} X \leqslant \omega^{\operatorname{dim}_{w m} X} .
$$

ТеОрема 4.48. Для того чтобы существовал $w$-m-C-компакт, топологически содержащий все $w-m-C$-компакты данного семейства $E$, необходимо и достаточно, чтобъ

$$
\sup \left\{\operatorname{dim}_{w m} X: X \in E\right\}<\omega_{1} .
$$

Пусть $(u, \Phi)=\left(\left\{U_{1}, \ldots, U_{k}\right\},\left\{F_{1}, \ldots, F_{k}\right\}\right)-m$-накрывающая пара и $O \Phi=$ $\left\{O F_{1}, \ldots, O F_{k}\right\}-$ окрестность $m$-системы $\Phi$, вписанная в $u$. Тогда множество $P=X \backslash O F_{1} \cup \cdots \cup O F_{k}$ называется перегородкой пары $(u, \Phi)$. 
ОПРЕДЕЛЕНиЕ 4.49. Большая трансфинитная индуктивная размерность $\operatorname{Ind}_{m}(m \geqslant 2$ или $m=\infty)$ в классе всех нормальных пространств определяется по индукции следующим образом.

1) $\operatorname{Ind}_{m} X=-1 \Longleftrightarrow X=\varnothing$.

2) $\operatorname{Ind}_{m} X \leqslant \alpha$, где $\alpha$ - порядковое число, если для всякой $m$-накрывающей пары $(u, \Phi)$ существует ее перегородка $P$ размерности $\operatorname{Ind}_{m} P<\alpha$.

3) $\operatorname{Ind}_{m} X=\infty$, если неравенство $\operatorname{Ind}_{m} X \leqslant \alpha$ не выполняется ни для какого порядкового числа $\alpha$.

В последнем случае будем говорить также, что размерность $\operatorname{Ind}_{m} X$ не определена.

Из определения 4.49 вытекают соотношения

$$
\text { Ind } X=\operatorname{Ind}_{2} X \leqslant \operatorname{Ind}_{3} X \leqslant \cdots \leqslant \operatorname{Ind}_{m} X \leqslant \cdots \leqslant \operatorname{Ind}_{\infty} X .
$$

Следующее утверждение достаточно очевидно.

ПРЕДЛОЖЕНИЕ 4.50. Пусть в пространстве $X$ выполнена теорема конечной суммы для замкнутых слагаемых конечной размерности Ind $\leqslant n-1$. Тогда Ind $F=\operatorname{Ind}_{\infty} F$ для всякого замкнутого множества $F \subset X$ размерности Ind $F \leqslant n$.

СлЕДСТвиЕ 4.51. Если Ind $X=0$, mo $\operatorname{Ind}_{\infty} X=0$.

ТЕОремА 4.52. Пусть наследственно нормальный бикомпакт $X$ является объединением своих подпространств $X_{i}, i \in \omega$, размерность $\operatorname{Ind}_{m} X_{i}$ которых определена. Тогда $\operatorname{Ind}_{m} X<\infty$.

Доказательство проходит по схеме доказательства теоремы 3 из [35]. При этом используется следующая стандартно доказываемая лемма.

Лемма 4.53. Пусть $Y$ - непустое подмножество наследственно нормального пространства $X$ размерности $\operatorname{Ind}_{m} Y=\alpha$. Тогда для произвольной $m$ накрывающей пары $(u, \Phi)$ пространства $X$ существует такая перегородка $P$ этой nарьь, что $\operatorname{Ind}_{m}(P \cap Y)<\alpha$.

Из следствия 4.51 и теоремы 4.52 вытекает следующее утверждение.

УТВЕРЖДЕНИЕ 4.54. Для любого счетномерного метризуемого компакта определены размерности $\operatorname{Ind}_{m}$ для всех $\mathrm{m}$.

ОПРЕДЕЛЕНИЕ 4.55. Семейство $\mathscr{U}=\left\{u_{\alpha}: \alpha \in A\right\} \subset \operatorname{cov}_{m}(X)$ называется существенным на $Y \subset X$, если для любых открытых дизъюнктных семейств $v_{\alpha}$, вписанных в $u_{\alpha}$, имеем $Y \backslash \bigcup\left\{\bigcup v_{\alpha}: \alpha \in A\right\} \neq \varnothing$.

Для $Y \subset X$ полагаем

$$
M_{m}(X, Y)=\left\{\sigma \in \operatorname{Fin}\left(\operatorname{cov}_{m}(X)\right): \sigma \text { существенно на } Y \text { и }|\sigma|=|\sigma| Y \mid\right\} .
$$

Обобщением теоремы 3.1.6 из [48] является следующее утверждение.

ПреДЛОЖЕНИЕ 4.56. Если $F$ - замкнутое подмножество пространства $X$, mo

$$
\operatorname{dim}_{m} F=\operatorname{Ord} M_{m}(X, F) \leqslant \operatorname{dim}_{m} X .
$$


ДокАзАтельство. Правое неравенство вытекает из (4.5). Чтобы проверить левое равенство, воспользуемся леммой 4.27. Положим

$$
L_{1}=\left\{e(u): u \in \operatorname{cov}_{m}(F)\right\}
$$

где $e$ - оператор из доказательства предложения 4.10;

$$
L_{2}=\operatorname{cov}_{m}(X) ; \quad M_{1}=\left\{e(\sigma): \sigma \in M_{m}(F)\right\} ; \quad M_{2}=M_{m}(X, F) .
$$

Проверим выполнение свойств 1)-3) леммы 4.27. Пусть $a_{2}=\left\{U_{1}, \ldots, U_{k}\right\} \in$ $L_{2} \cap M_{2}$. Согласно (4.10), покрытие $a_{2}$ существенно на $F$. Это означает, что в семейство $a_{2} \mid F=\left\{U_{1} \cap F, \ldots, U_{k} \cap F\right\}$ нельзя вписать дизъюнктное покрытие множества $F$. Возьмем последовательность покрытий $w_{i}=\left\{W_{1}^{i}, \ldots, W_{k}^{i}\right\}$, $i \in \omega$, множества $F$, удовлетворяющих условиям:

$$
\left[W_{j}^{0}\right] \subset U_{j} \cap F ; \quad\left[W_{j}^{i+1}\right] \subset W_{j}^{i} .
$$

Покрытия $w_{i}$ существенны, будучи измельчениями существенного покрытия $a_{2} \mid F$. Из их существенности и условий (4.11) вытекает, что все они различны и отличаются от $a_{2} \mid F$. Покажем, что покрытия $e\left(w_{i}\right)$ годятся на роль элементов $a_{1} \in L_{1} \cap M_{1}$ из свойства 4.27.1).

Пусть $\sigma=\left\{u_{1}, \ldots, u_{s}\right\} \in M_{2}^{a_{2}}\left[\overline{e\left(w_{i}\right)}\right]$. Это означает, что

$$
\left\{a_{2}, u_{1}, \ldots, u_{s}\right\} \in M_{m}(X, F)
$$

и

$$
e\left(w_{i}\right) \notin\left\{a_{2}, u_{1}, \ldots, u_{s}\right\}
$$

Доказать же надо, что

$$
\left\{e\left(w_{i}\right), u_{1}, \ldots, u_{s}\right\} \in M_{m}(X, F) .
$$

Но это очевидно, поскольку семейство (4.13) получается из (4.12) заменой покрытия $\alpha_{2}$ на такое покрытие $e\left(w_{i}\right)$, что $e\left(w_{i}\right)\left|F=w_{i} \succ \alpha_{2}\right| F$.

Теперь проверим 4.27.2). Пусть $M_{2}^{\sigma} \neq \varnothing$. Тогда существует такое покрытие $u \in L_{2} \backslash \sigma$, что $\{u\} \in M_{2}^{\sigma}$. Если $u=\left\{U_{1}, \ldots, U_{k}\right\}$, то у последовательности $w_{i}, i \in \omega$, удовлетворяющей условиям (4.11), найдется такое $w_{i}$, что $e\left(w_{i}\right) \notin e(\sigma)$. Тогда $\left\{e\left(w_{i}\right)\right\} \in M_{1}^{\sigma}$. Если же $M_{1}^{\sigma} \neq \varnothing$, то существует такое существенное покрытие $u \in \operatorname{cov}_{m}(F)$, что $\{e(u)\} \in M_{1}^{\sigma}$. В этом случае автоматически $\{e(u)\} \in M_{2}^{\sigma}$.

Наконец, условие 4.27.3) выполнено тривиальным образом.

Предложение 4.56 доказано.

Аналогом предложения 3.2.1 из [48] является следующее утверждение.

Лемма 4.57. Пусть $\left(u_{1}, \Phi_{1}\right)$ - т-накрывающая пара пространства $X$ и $P$ ее перегородка. Тогда

$$
\left(M_{m}(X)\right)^{u_{1}} \subset M_{m}(X, P)
$$

u, значит,

$$
\operatorname{Ord}\left(M_{m}(X)\right)^{u_{1}} \leqslant \operatorname{dim}_{m} P
$$


Доказательство. Пусть $\sigma=\left\{u_{2}, \ldots, u_{s}\right\} \in\left(M_{m}(X)\right)^{u_{1}}$. Тогда по определению $\left\{u_{1}, u_{2}, \ldots, u_{s}\right\} \in M_{m}(X)$. Следовательно, для любых открытых дизъюнктных семейств $v_{i}$, вписанных в $u_{i}$, семейство

$$
\bigcup\left\{v_{i}: i=1, \ldots, s\right\} \quad \text { не покрывает } X \text {. }
$$

В частности, свойство (4.16) выполнено и для семейства $v_{1}=O \Phi_{1}$, где $P=$ $X \backslash \bigcup O \Phi_{1}$. Поэтому семейство $\bigcup\left\{v_{2}, \ldots, v_{s}\right\}$ не покрывает $P$. Но это и означает, что $\left\{u_{2}, \ldots, u_{s}\right\}$ существенно на $P$, т.е. $\sigma \in M_{m}(X, P)$. Итак, свойство (4.14) проверено. Свойство (4.15) вытекает из (4.14) и предложения 4.56. Лемма 4.57 доказана.

Следующее утверждение для $m=2$ было доказано в [48].

Теорема 4.58. Пусть $X$ - нормальное пространство и $m \geqslant 2$ или $m=\infty$. Тогда

$$
\operatorname{dim}_{m} X \leqslant \operatorname{Ind}_{m} X .
$$

ДоказАтельСтво. Индукция по $\operatorname{Ind}_{m} X=\alpha$. Случай $\alpha=-1$ очевиден. Пусть $\operatorname{Ind}_{m} X=\alpha \geqslant 0$. Берем произвольное $m$-покрытие $u=\left\{U_{1}, \ldots, U_{k}\right\}$. Ему соответствует $m$-накрывающая пара $(u, \Phi)$, где $m$-система $\Phi$ состоит из пустых множеств. Поскольку $\operatorname{Ind}_{m} X=\alpha$, существуют такие попарно непересекающиеся открытые множества $V_{i} \subset U_{i}$, что $\operatorname{Ind}_{m} P<\alpha$ для $P=X \backslash V_{1} \cup \cdots \cup V_{k}$. Согласно предположению индукции, $\operatorname{dim}_{m} P \leqslant \operatorname{Ind}_{m} P$. Тогда из леммы 4.57 получаем, что $\operatorname{Ord}\left(M_{m}(X)\right)^{u}<\alpha$. Следовательно, $\operatorname{Ord}\left(M_{m}(X)\right) \leqslant \alpha$. Teоpeма 4.58 доказана.

П. Борст [52] привел пример метризуемого компакта $X$ с размерностями

$$
\operatorname{dim}_{2} X=\omega_{0}<\operatorname{Ind}_{2} X=\omega_{0}+1 .
$$

Следующие два утверждения явялются аналогами соответствующих теорем Ю. М. Смирнова (см. [22], [42]) для размерности Ind.

Теорема 4.59. Если для бикомпакта $X$ веса $w X \leqslant \omega_{\alpha}$ определена размерность $\operatorname{Ind}_{m}$, mo $\operatorname{Ind}_{m} X<\omega_{\alpha+1}$.

Теорема 4.60. Если для метрического пространства $Х$ определена размерность $\operatorname{Ind}_{m}$, mо $\operatorname{Ind}_{m} X<\omega_{1}$.

\section{§ 5. Факторизационные теоремы и компактификации}

ОПРеДЕЛЕНИЕ 5.1. Отображение $f: X \rightarrow Y$ назовем $m$-существенно накрывающим, если $f^{-1}\left(M_{m}(Y)\right) \subset M_{m}(X)$, т.е. для всякого существенного семейства $\left(u_{1}, \ldots, u_{k}\right) \in \operatorname{Fin}\left(\operatorname{cov}_{m}(X)\right)$ семейство $\left(f^{-1}\left(u_{1}\right), \ldots, f^{-1}\left(u_{k}\right)\right)$ существенно.

Аналогично, $f: X \rightarrow Y$ называется $w$-m-существенно накрывающим, если $f^{-1}\left(L_{m}(Y)\right) \subset L_{m}(X)$.

ПреДЛОЖЕНИЕ 5.2. Пусть $f: X \rightarrow Y-m$-существенно (w-m-существенно) накрывающее отображение. Тогда если $X \in m-C \quad(X \in w-m-C)$, то $Y \in m-C(Y \in w-m-C)$. Более того, $\operatorname{dim}_{m} Y \leqslant \operatorname{dim}_{m} X\left(\operatorname{dim}_{w m} Y \leqslant \operatorname{dim}_{w m} X\right)$. 
Утверждение вытекает из теорем 4.21, 4.41 и леммы 4.18, условиям которой удовлетворяют отображения

$$
\mu=f^{-1}: \operatorname{cov}_{m}(Y) \rightarrow \operatorname{cov}_{m}(X)
$$

и

$$
\mu=f^{-1}: \varphi_{m}(Y) \rightarrow \varphi_{m}(X) .
$$

Теорема 5.3 (факторизационная теорема для $\operatorname{dim}_{m}$ ). Пусть $f: X \rightarrow Z-$ отображение бикомпакта $X$ на бикомпакт $Z$. Тогда существуют такой бикомпакт $Y$ и такие отображения $g: X \rightarrow Y u h: Y \rightarrow Z$, что $f=h \circ g$, $\operatorname{dim}_{m} Y \leqslant \operatorname{dim}_{m} X u w Y=w Z$.

Аналогично формулируется

Теорема 5.4 (факторизационная теорема для $\operatorname{dim}_{w m}$ ). Пусть $f: X \rightarrow Z-$ отображение бикомпакта $X$ на бикомпакт $Z$. Тогда существуют такой бикомпакт $Y$ и такие отображения $g: X \rightarrow Y u h: Y \rightarrow Z$, что $f=h \circ g$, $\operatorname{dim}_{w m} Y \leqslant \operatorname{dim}_{w m} X u w Y=w Z$.

Доказательства теорем 5.3 и 5.4 проходят по одной схеме (поэтому мы ограничимся теоремой 5.3). Отображение $f$ надо разложить в $\tau$-спектр $S=\left\{X_{\alpha}\right.$, $\left.\pi_{\beta}^{\alpha}, A\right\}$, где $\tau=w Z$ (детали см. ниже), найти такое $\alpha$, что предельная проекция $\pi_{\alpha}: X \rightarrow X_{\alpha}$ является $m$-существенно накрывающей, положить $Y=X_{\alpha}$, $g=\pi_{\alpha}$ и применить предложение 5.2.

Спектр $S$. Положим $\kappa=w X$ и $A_{1}=\{\alpha \subset \kappa:|\alpha|=\tau\}$.

Множество $A_{1}$ упорядочим отношением включения: $\beta \leqslant \alpha \Longleftrightarrow \beta \subset \alpha$. Вложим $X$ в тихоновский куб $I^{\kappa}$. Положим $X_{\alpha}=p_{\alpha}(X), \pi_{\beta}^{\alpha}=p_{\beta}^{\alpha} \mid X_{\alpha}$, где $p_{\alpha}: I^{\kappa} \rightarrow I^{\alpha}$ и $p_{\beta}^{\alpha}: I^{\alpha} \rightarrow I^{\beta}-$ проектирования на грани. Вложение $X \rightarrow I^{\kappa}$ можно выбрать так, что для некоторого $\alpha_{0} \in A_{1}$ отображение $\pi_{\alpha_{0}}: X \rightarrow X_{\alpha_{0}}$ гомеоморфно отображению $f: X \rightarrow Z$. Полагаем $A=\left\{\alpha \in A_{1}: \alpha_{0} \leqslant \alpha\right\}$ и $S=\left\{X_{\alpha}, \pi_{\beta}^{\alpha}, A\right\}$. Тогда $X=\lim S$, а отображения $\pi_{\alpha}: X \rightarrow X_{\alpha}$ являются предельными проекциями $\tau$-спектра $S$.

Поиск существенно накрывающих отображений. (а) Отметим прежде всего, что если семейство $\left(v_{1}, \ldots, v_{n}\right)$ вписано в существенное семейство $\left(u_{1}, \ldots, u_{n}\right)$, то оно также существенно. Поэтому условие существенной накрываемости достаточно проверить для множества

$$
M_{m}^{0}(Y)=\left\{\sigma \in \operatorname{Fin}\left(\operatorname{cov}_{m}^{0}(Y)\right): \sigma \text { существенно }\right\},
$$

где $\operatorname{cov}_{m}^{0}(Y)$ - семейство покрытий, конфинальное в $\operatorname{cov}_{m}(Y)$. В качестве $\operatorname{cov}_{m}^{0}(Y)$ можно взять множество $\operatorname{cov}_{m}\left(Y, \mathscr{T}_{0}\right)$ всех $m$-покрытий, элементы которых принадлежат большой базе $\mathscr{T}_{0} \subset \mathscr{T}(Y)$ пространства $Y$ с $\left|\mathscr{T}_{0}\right|=w Y$.

(b) Для пространства $Y$ положим

$$
I_{m}(Y)=\left\{\sigma \in \operatorname{Fin}\left(\operatorname{cov}_{m}(Y)\right): \sigma \text { несущественно }\right\} .
$$

Если $f: X \rightarrow Y$ - отображение, а $\mathscr{T}_{0} \subset \mathscr{T}(Y)$, то

$$
I_{m}(f)\left(\mathscr{T}_{0}\right)=\left\{\sigma \in \operatorname{Fin}\left(\operatorname{cov}_{m}\left(Y, \mathscr{T}_{0}\right)\right): f^{-1}(\sigma) \text { несущественно }\right\} .
$$


Отображение $f \quad m$-существенно накрывающее тогда и только тогда, когда существует такая большая база $\mathscr{T}_{0} \subset \mathscr{T}(Y)$, что

$$
I_{m}(f)\left(\mathscr{T}_{0}\right) \subset I_{m}(Y) .
$$

(c) Очевидно, что если $X$ есть предел спектра $S=\left\{X_{\alpha}, \pi_{\beta}^{\alpha}, A\right\}$ и $\sigma \in I_{m}(X)$, то существует такое $\alpha \in A$, что $\pi_{\alpha}^{\#} \sigma \in I_{m}\left(X_{\alpha}\right)$.

(d) Приступаем к поиску. Фиксируем большую базу $\mathscr{T}$ бикомпакта $X_{\alpha_{0}}=Z$ мощности $\tau$. Для каждого $\sigma \in I_{m}\left(\pi_{\alpha_{0}}\right)\left(\mathscr{T}_{0}\right)$ согласно (c) существует такое $\alpha(\sigma) \in A$, что

$$
\pi_{\alpha(\sigma)}^{\#} \pi_{\alpha_{0}}^{-1}(\sigma) \in I_{m}\left(X_{\alpha(\sigma)}\right) .
$$

Существует элемент $\alpha_{1} \in A$, мажорирующий все $\alpha(\sigma)$. Тогда

$$
\pi_{\alpha_{1}}^{\#} \pi_{\alpha_{0}}^{-1}(\sigma) \in I_{m}\left(X_{\alpha_{1}}\right) \quad \forall \sigma \in I_{m}\left(\pi_{\alpha_{0}}\right)\left(\mathscr{T}_{0}\right) .
$$

Семейство $\left(\pi_{\alpha_{0}}^{\alpha_{1}}\right)^{-1}\left(\mathscr{T}_{0}\right)$ дополняем до большой базы $\mathscr{T}_{1}$ бикомпакта $X_{\alpha_{1}}$ мощности $\tau$. Повторяя описанную выше процедуру, находим такое $\alpha_{2}>\alpha_{1}$, что

$$
\pi_{\alpha_{2}}^{\#} \pi_{\alpha_{1}}^{-1}(\sigma) \in I_{m}\left(X_{\alpha_{2}}\right) \quad \forall \sigma \in I_{m}\left(\pi_{\alpha_{1}}\right)\left(\mathscr{T}_{1}\right) .
$$

Поступая аналогичным образом, находим такую растущую последовательность элементов $\alpha_{i} \in A$, что

$$
\pi_{\alpha_{i+1}}^{\#} \pi_{\alpha_{i}}^{-1}(\sigma) \in I_{m}\left(X_{\alpha_{i+1}}\right) \quad \forall \sigma \in I_{m}\left(\pi_{\alpha_{i}}\right)\left(\mathscr{T}_{i}\right)
$$

где $\mathscr{T}_{i}$ - большая база бикомпакта $X_{\alpha_{i}}$, содержащая семейство $\left(\pi_{\alpha_{i-1}}^{\alpha_{i}}\right)^{-1}\left(\mathscr{T}_{i-1}\right)$.

(е) Полагаем $\alpha=\lim \alpha_{i}$,

$$
\mathscr{T}^{0}=\bigcup\left\{\left(\pi_{\alpha_{i}}^{\alpha}\right)^{-1}\left(\mathscr{T}_{i}\right): i \in \omega\right\} .
$$

Тогда $\mathscr{T}^{0}$ будет большой базой бикомпакта $X_{\alpha} \equiv Y$, удовлетворяющей условию

$$
I_{m}\left(\pi_{\alpha}\right)\left(\mathscr{T}^{0}\right) \subset I_{m}(Y) .
$$

Факторизационная теорема 5.3 доказана.

Для пространства $Y$ положим

$$
\begin{gathered}
\varphi_{m}^{0}(Y)=\left\{\Phi \in \varphi_{m}(Y): \text { элементы } \Phi \text { канонически замкнуты }\right\} \\
M_{w m}^{0}(Y)=M_{w m}(Y) \cap \operatorname{Fin} \varphi_{m}^{0}(Y) .
\end{gathered}
$$

ЛЕмма 5.5. Для пространства $Y$ имеем

$$
\operatorname{dim}_{w m} Y=\operatorname{Ord} M_{w m}^{0}(Y) .
$$

ДоказАтельство. Тождественное вложение $M_{w m}^{0}(Y) \rightarrow M_{w m}(Y)$ дает неравенство Ord $M_{w m}^{0}(Y) \leqslant \operatorname{dim}_{w m} Y$. Теперь для $\Phi=\left\{F_{1}, \ldots, F_{k}\right\} \in \varphi_{m}(Y)$ зафиксируем такую систему $\mu(\Phi)=\left\{F_{1}^{\prime}, \ldots, F_{k}^{\prime}\right\} \in \varphi_{m}^{0}(Y)$, что $F_{i} \subset F_{i}^{\prime}$. Получаем отображение $\mu: \varphi_{m}(Y) \rightarrow \varphi_{m}^{0}(Y)$, удовлетворяющее условию

$$
\mu\left(M_{w m}(Y)\right) \subset M_{w m}^{0}(Y) .
$$


Более того, если $\sigma=\left\{\Phi_{1}, \ldots, \Phi_{n}\right\} \in L_{m}(Y)$, то для $\mu(\sigma)=\left\{\mu\left(\Phi_{1}\right), \ldots, \mu\left(\Phi_{n}\right)\right\}$ имеем $|\sigma|=|\mu(\sigma)|$, поскольку семейство $\mu(\sigma)$, содержащее две одинаковые системы, не может быть существенным. Таким образом, отображение $\mu$ удовлетворяет условиям леммы 4.18 , согласно которой $\operatorname{Ord} M_{w m}(Y) \leqslant \operatorname{Ord} M_{w m}^{0}(Y)$. Лемма 5.5 доказана.

ТеоремА 5.6. Для нормального пространства $X$ имеем

$$
\operatorname{dim}_{w m} X=\operatorname{dim}_{w m} \beta X .
$$

ДоказАтельство. Если $F \subset \beta X$ - канонически замкнутое множество, то $F=[F \cap X]_{\beta X}$. Наоборот, если $F \subset X$ канонически замкнуто, то $F=[F]_{\beta X} \cap X$. Поэтому с учетом нормальности $X$ отображение

$$
\left\{F_{1}, \ldots, F_{k}\right\} \rightarrow\left\{\left[F_{1}\right]_{\beta X}, \ldots,\left[F_{k}\right]_{\beta X}\right\}
$$

порождает изоморфизм

$$
\operatorname{Ord} M_{w m}^{0}(X) \rightarrow \operatorname{Ord} M_{w m}^{0}(\beta X) .
$$

Применение леммы 5.5 завершает доказательство.

Для пространства $Y$ положим

$$
\begin{aligned}
\operatorname{cov}_{m}^{0}(Y) & =\left\{u \in \operatorname{cov}_{m}(Y): \text { элементы } u \text { канонически открыты }\right\} ; \\
M_{m}^{0}(Y) & =M_{m}(Y) \cap \operatorname{Fin}\left(\operatorname{cov}_{m}^{0}(Y)\right) .
\end{aligned}
$$

ЛЕмма 5.7. Для пространства $Y$ имеем

$$
\operatorname{dim}_{m} Y=\operatorname{Ord} M_{m}^{0}(Y) .
$$

ДокАзАТЕЛьство. Достаточно показать, что пары

$$
\begin{aligned}
\left(\operatorname{cov}_{m}^{0}(Y), \operatorname{cov}_{m}(Y)\right) & =\left(L_{1}, L_{2}\right), \\
\left(M_{m}^{0}(Y), M_{m}(Y)\right) & =\left(M_{1}, M_{2}\right)
\end{aligned}
$$

удовлетворяют условиям леммы 4.27. Очевидно, $M_{m}^{0}(Y), M_{m}(Y)$ - включающие множества. Для любого существенного покрытия $u \equiv a_{2} \in \operatorname{cov}_{m}(Y)$ в качестве элементов $a_{1}$ берем бесконечную последовательность канонически открытых покрытий

$$
u_{1} \prec u_{2} \prec \cdots,
$$

где каждое следующее комбинаторно с замыканием вписано в предыдущее (включая вписанность $u \prec u_{1}$ ). Из существенности $u$ вытекает существенность $u_{i}$ и их попарная различность. После этого проверка условия 1) состоит в расшифровке определений.

Для открытого множества $U$ через $\bar{U}$ обозначим его "канонизацию": $\bar{U}=$ $\operatorname{Int}[U]$. На основе этого определяется "канонизация" $\bar{u}$ покрытия $u$ и "канонизация" $\bar{\sigma}$ семейства покрытий $\sigma$. Ясно, что

$$
\sigma \in M_{m}(Y) \Longleftrightarrow \bar{\sigma} \in M_{m}^{0}(Y) .
$$

Импликация $\Longrightarrow$ из (5.4) дает нам условие 2) леммы 4.27. Условие 3) очевидно. Лемма 5.7 доказана. 
Теорема 5.8. Для нормального пространства $X$ имеем

$$
\operatorname{dim}_{m} X=\operatorname{dim}_{m} \beta X .
$$

ДоказАтельство. Для канонически открытого в $X$ множества $U$ множество $\operatorname{ex}(U) \equiv \beta X \backslash[X \backslash U]_{\beta} X$ канонически открыто в $\beta X$. Для покрытия $u=\left\{U_{1}, \ldots, U_{k}\right\}$ пространства $X$ семейство $\operatorname{ex}(u)=\left\{\operatorname{ex}\left(U_{1}\right), \ldots, \operatorname{ex}\left(U_{k}\right)\right\}$ является покрытием $\beta X$ в силу нормальности $X$. Легко видеть, что

$$
\sigma \in M_{m}^{0}(X) \Longleftrightarrow \operatorname{ex}(\sigma) \in M_{m}^{0}(\beta X) .
$$

Из (5.5) получаем $\operatorname{Ord} M_{m}^{0}(X)=\operatorname{Ord} M_{m}^{0}(\beta X)$. Применение леммы 5.7 завершает доказательство.

Из теорем 4.21, 5.8 и 5.3 вытекает

Tеорема 5.9. Всякое $S$-m-C-пространство $X$ имеет $m$-C-компактификацию $b X$ веса $w b X=w X$ и размерности $\operatorname{dim}_{m} b X \leqslant \operatorname{dim}_{m} X$.

Из теорем 4.41, 5.6 и 5.4 вытекает

Tеорема 5.10. Всякое $S$-w-m-C-пространство $X$ имеет $w$-m-C-компактификачию $b X$ веса $w b X=w X$ и размерности $\operatorname{dim}_{w m} b X \leqslant \operatorname{dim}_{w m} X$.

Изложенные выше результаты для $m=2$ получили К. Иокои [53] и В. А. Чатырко [54], а для $m=\infty-$ В. А. Чатырко [55], [56].

ЗАмечаниЕ 5.11. Распространить утверждения теорем 5.9 и 5.10 на $m-C$ - и $w$ - $m$ - $C$-пространства нельзя. В самом деле, обозначим через $Q_{\omega}$ подмножество гильбертова куба $Q=I^{\omega}$, состоящее из всех точек, лишь конечное число координат которых отлично от нуля. Пространство $Q_{\omega}$ слабо бесконечномерно и даже является $C$-пространством, но, как показал Е. Г. Скляренко [5], не имеет слабо бесконечномерных компактификаций.

Напомним, что счетная последовательность открытых множеств $\Gamma_{n}, n \in \omega$, пространства $X$ называется сходящейся, если для всякой дискретной в $X$ последовательности точек $x_{i}, i \in \omega$, существуют такие $i_{0}$ и $n$, что $x_{i} \in \Gamma_{n}$ для всех $i \geqslant i_{0}$. Если последовательность $\Gamma_{n}$ сходится, то множество $\Phi=X \backslash \bigcup\left\{\Gamma_{n}: n \in \omega\right\}$ называется пределом этой последовательности. Ясно, что предел всякой сходящейся последовательности счетно компактен.

Обобщением теоремы Е. Г. Скляренко о строении $S$-слабо бесконечномерных пространств (см. [5], [46]) является следующая теорема.

ТеОРема 5.12. Наследственно паракомпактное пространство $X$ является $S-m-C$ (S-w-m-C)-пространством тогда и только тогда, когда в X существует сходящаяся последовательность открытых конечномерных множеств $\Gamma_{n}$, предел которой есть m-C (w-m-C)-бикомпакт.

Для $S$-m- $C$-пространств эта теорема доказана в [15].

ТеОрема 5.13. Пусть $X$ - полное метрическое пространство, каждое компактное подмножество которого есть $m-C$ (w-m-C)-пространство. Тогда существует бикомпактное расширение $b X$, такюе являющееся $m-C$ $(w-m-C)$-пространством и имеющее вес $w b X=w X$. 
СЛЕДСТВИЕ 5.14. Всякое полное метрическое $m-C$ (w-m-C)-пространство имеет m-C (w-m-C)-компактификацию.

СлЕДСТВИЕ 5.15. Всякое полное метрическое $C(w-C)$-пространство имеет $C(w-C)$-компактификацию.

С учетом предложения 1.34 и замечаний 1.35 и 1.36 теорема 5.13 вытекает из следующего утверждения.

Теорема 5.16 [57]. Всякое полное метрическое пространство X имеет бикомпактное расширение в $X$ веса $w X$, нарост которого является объединением счетного числа конечномерных бикомпактов.

На трансфинитную размерность $\operatorname{Ind}_{m}$ распространяются результаты о размерности Ind $=\operatorname{Ind}_{2}$, полученные Б. А. Пасынковым в [58].

Теорема 5.17. Для произволъного нормалъного пространства $X$

$$
\operatorname{Ind}_{m} X=\operatorname{Ind}_{m} \beta X .
$$

Теорема 5.18. Если $\operatorname{Ind}_{m} \beta X \leqslant \alpha$ для тихоновского пространства $X$, то существует такое бикомпактное расширение $b X$, что

$$
w b X=w X, \quad \operatorname{Ind}_{m} b X \leqslant \alpha .
$$

Теорема 5.18 вытекает из следующей факторизационной теоремы.

Теорема 5.19. Пусть $f: X \rightarrow Z$ - отображение между бикомпактами, $u$ пусть $F \subset X$ - замкнутое множество размерности $\operatorname{Ind}_{m} F \leqslant \alpha$. Тогда существуют такой бикомпакт $Y$ и такие непрерывные отображения $g: X \rightarrow Y u$ $h: Y \rightarrow Z$, что

1) $f=h \circ g$;

2) $w Y \leqslant w Z$

3) $\operatorname{Ind}_{m} f(F) \leqslant \alpha$.

Доказательство этой теоремы проходит по схеме доказательства теоремы 3 из [17; приложение].

Из теорем 5.17 и 5.18 вытекает следующее утверждение.

СлЕДСТвИЕ 5.20. Для нормального пространства X существует такое бикомпактное расширение $b X$, что $w b X=w X u \operatorname{Ind}_{m} b X \leqslant \operatorname{Ind}_{m} X$.

ЗАмЕчАниЕ 5.21. Существуют нульмерные бикомпакты, содержащие нормальные подпространства $X$ положительной размерности Ind $X$ (первый такой пример построил Даукер [59]). Значит, равенство размерностей не является необходимым в следствии 5.20.

\section{§ 6. Существенные отображения и $m$-компакты Хендерсона}

Основным инструментом исследования $m-C$ - и $w$ - $m$ - $C$-пространств являются $k$-оды и их произведения. Пусть $A_{k}=\left\{a_{1}, \ldots, a_{k}\right\}$ - конечное множество, состоящее из $k \geqslant 2$ точек. Конус $\operatorname{con}\left(A_{k}\right)$ называется $k$-одом. По другому $k$ од можно описать как простейшее дерево $T(k)$ с $k$ вершинами и (при $k \geqslant 3$ ) 
единственной точкой ветвления - вершиной конуса $\operatorname{con}\left(A_{k}\right)$, которая будет называться иентром $T(k)$ и обозначаться через $c$.

Компакт $T(k)$ можно рассматривать как объединение $k$ радиусов $\left[c ; a_{j}\right]$ единичной окружности $S^{1} \subset \mathbb{R}^{2}$ с центром в точке $c$. Отрезки $\left[c ; a_{j}\right]$ называются ребрами $k$-ода $T(k)$, а точки $a_{j}$ - его вершинами. При рассмотрении нескольких $k$-одов с различным числом вершин центр $k$-ода $T(k)$ будем обозначать через $c_{k}$, а его вершины $-a_{1}^{k}, \ldots, a_{k}^{k}$. Каждый $k$-од, очевидно, является абсолютным ретрактом.

Пусть $\kappa=\left(k_{1}, \ldots, k_{i}, \ldots\right)-m$-последовательность, а $\kappa_{n}=\left(k_{1}, \ldots, k_{n}\right)-$ ее начальный отрезок. Положим

$$
\begin{aligned}
T_{\kappa} & =\Pi\left\{T\left(k_{i}\right): k_{i} \in \kappa\right\}, \\
T_{\kappa_{n}} & =\Pi\left\{T\left(k_{i}\right): k_{i} \in \kappa_{n}\right\} .
\end{aligned}
$$

Иногда, если последовательность $\kappa$ фиксирована, мы будем применять более простые обозначения: $T_{\kappa}=T, T_{\kappa_{n}}=T_{n}$.

Через

$$
q_{i}: T \rightarrow T\left(k_{i}\right), \quad q^{n}: T \rightarrow T_{n}, \quad q_{i}^{n}: T_{n} \rightarrow T\left(k_{i}\right)
$$

мы будем обозначать проектирования произведения на сомножитель и на подпроизведение соответственно. Ясно, что $q^{n}$ совпадает с диагоналъным произведением отображений $q_{i}$ :

$$
q^{n}=\underset{k_{i} \in \kappa_{n}}{\triangle} q_{i}
$$

Положим

$$
\Gamma_{j}^{i}=\left\{t=\left(t_{l}\right) \in T: t_{i}=a_{j}^{k_{i}}\right\} .
$$

Множество $\Gamma_{j}^{i}$ будем называть $(i, j)$-гранъю компакта $T$. Аналогично определяется $(i, j)$-грань ${ }^{n} \Gamma_{j}^{i}$ компакта $T_{n}$ при $i \leqslant n$ :

$$
{ }^{n} \Gamma_{j}^{i}=\left\{t=\left(t_{l}\right) \in T_{n}: t_{i}=a_{j}^{k_{i}}\right\}=\left(q_{i}^{n}\right)^{-1}\left(a_{i}^{k_{i}}\right) .
$$

Множество

$$
\partial T_{n}=\bigcup\left\{{ }^{n} \Gamma_{j}^{i}: i \leqslant n, j \leqslant k_{i}\right\}
$$

называется комбинаторной границей компакта $T_{n}$. Можно показать, что

$$
\text { компакт } T_{n} \text { гомеоморфен конусу } \operatorname{con}\left(\partial T_{n}\right) \text {. }
$$

Если последовательность $\kappa$ постоянна: $\kappa=\{k, k, \ldots, k, \ldots\}$, то компакты $T_{n}$ являются степенями $k$-ода $T(k)$ и обозначаются $T^{n}(k)$.

Следующее утверждение заменяет собой лемму Урысона при исследовании $m-C$ - и $w-m-C$-пространств.

ПРедлОЖЕНИЕ 6.1 (лемма о продолжении отображений в $k$-оды). Пусть $\Phi=\left\{F_{1}, \ldots, F_{k}\right\}-$ дизгюнктная система замкнутых подмножеств пространства $X, O \Phi=\left\{O F_{1}, \ldots, O F_{k}\right\}$ - ее окрестность. Тогда существует такое непрерывное отображение $f: X \rightarrow T(k)$, что $f\left(F_{j}\right)=a_{j}^{k} u f^{-1}\left(c_{k} ; a_{j}^{k}\right] \subset O F_{j}$. Если, кроме того, перегородка $P=X \backslash O F_{1} \cup \cdots \cup O F_{k}$ и множества $F_{j}$ функционально замкнуты, то можно предполагать, что

$$
F_{j}=f^{-1}\left(a_{j}^{k}\right), \quad P=f^{-1}\left(c_{k}\right) .
$$


ОПРеДЕЛЕНИЕ 6.2. Для $m \in \overline{\mathbb{N}}$ и $m$-последовательности $\kappa_{n}=\left(k_{1}, \ldots, k_{n}\right)$ непрерывное отображение $f: X \rightarrow T_{\kappa_{n}}$ называется $w$-несущественным, если существует непрерывное отображение $g: X \rightarrow \partial T_{\kappa_{n}}$, совпадающее с $f$ на множестве $f^{-1}\left(\partial T_{\kappa_{n}}\right)$.

ОПРЕДЕЛЕНИЕ 6.3. Для $m \in \overline{\mathbb{N}}$ и $m$-последовательности $\kappa=\left\{k_{i}: i \in \mathbb{N}\right\}$ непрерывное отображение $f: X \rightarrow T_{\kappa}$ называется $w$-существенным, если для всякого $n \geqslant 1$ отображение

$$
q^{n} \circ f: X \rightarrow T_{\kappa_{n}}
$$

w-существенно.

Теорема 6.4. Для $m \in \overline{\mathbb{N}}$ нормальное пространство $X$ является $S-w-m$ $C$-пространством тогда и только тогда, когда выполнено условие

(*) для любой $m$-последовательности $\kappa=\left\{k_{i}: i \in \mathbb{N}\right\}$ всякое отображение $f: X \rightarrow T_{\kappa} w$-несущественно.

Для $m=2$ теорему 6.4 доказал Б. Т. Левшенко [4].

СледСтвиЕ 6.5. Бикомпакт $X$ является $w$-C-пространством тогда $u$ только тогда, когда для любой последовательности $\kappa=\left(k_{i}\right), k_{i} \geqslant 2$, всякое отображение $f: X \rightarrow T_{\kappa} w$-несущественно.

Доказательство теоремы 6.4 основано на (6.4) и следующих двух утверждениях, представляющих самостоятельный интерес.

Лемма 6.6. Пусть $\sigma=\left\{\Phi_{1}, \ldots, \Phi_{n}\right\}, \Phi_{i}=\left(F_{1}^{i}, \ldots, F_{k_{i}}^{i}\right),-$ существенное семейство систем замкнутых множеств пространства $X$. Тогда $w$-существенно всякое отображение

$$
f=\triangle_{i=1}^{n} f_{i}: X \rightarrow T\left(k_{1}\right) \times \cdots \times T\left(k_{n}\right),
$$

подчиненное условиям $f_{i}^{-1}\left(a_{j}^{k_{i}}\right)=F_{j}^{i}$.

ЛЕмма 6.7. Если w-существенно отображение

$$
f=\triangle_{i=1}^{n} f_{i}: X \rightarrow T\left(k_{1}\right) \times \cdots \times T\left(k_{n}\right),
$$

то в $X$ существенно семейство $\sigma=\left\{\Phi_{1}, \ldots, \Phi_{n}\right\}$, где

$$
\Phi_{i}=\left(f_{i}^{-1}\left(a_{1}^{k_{i}}\right), \ldots, f_{i}^{-1}\left(a_{k_{i}}^{k_{i}}\right)\right) .
$$

В 1968 г. Д. Хендерсон [60] для $\alpha<\omega_{1}$ определил AR-компакты $J^{\alpha}$ размерности Ind $J^{\alpha}=\alpha$, их границы $\partial J^{\alpha}=T^{\alpha}$ и существенные отображения $f: X \rightarrow J^{\alpha}$. Он доказал, что если нормальное пространство $X$ допускает существенное отображение на $J^{\alpha}$, то Ind $X \geqslant \alpha$. Там же Хендерсон поставил два вопроса:

1) Допускает ли $X$ существенное отображение на $J^{\alpha}$, если Ind $X \geqslant \alpha$ ?

2) Будет ли сильно бесконечномерным метризуемый компакт $X$, если он допускает существенное отображение на каждый компакт $J^{\alpha}, \alpha<\omega_{1}$ ? 
Обе проблемы решил Р. Поль [47]. Положительный ответ на второй вопрос вытекает из того, что index $X \geqslant \alpha$, если $X$ допускает существенное отображение на $J^{\alpha}$. Отрицательно отвечая на первый вопрос, Р. Поль доказал существование слабо бесконечномерного компакта, который содержит подкомпакты любой трансфинитной размерности $<\omega_{1}$.

П. Борст и Я. Дийкстра [61] построили $\sigma$-конечномерный компакт $X$ размерности Ind $X=\omega_{0}+1$, который не допускает существенного отображения на $J^{\omega_{0}+1}$. При этом оказалось, что $\operatorname{dim}_{2} X=\omega_{0}$. Затем Борст построил пример [53], показывающий, что неравенство $\operatorname{dim} Y \geqslant \alpha$ не является достаточным для того, чтобы компакт $Y$ допускал существенное отображение на $J^{\alpha}$. В той же статье [52] он получил исчерпывающие результаты, характеризующие размерность $\operatorname{dim}_{2}$ в терминах существенных отображений на компакты Хендерсона. Все эти результаты переносятся на размерность $\operatorname{dim}_{w m}$. Приводимые ниже утверждения о размерности $\operatorname{dim}_{w m}$ при $m=2$ превращаются в теоремы Борста. Начнем мы с обобщения конструкции Хендерсона.

ОПРЕДЕЛЕНиЕ 6.8. Фиксируем целое $m \geqslant 2$. Для всякого трансфинита $\alpha<\omega_{1}$ по индукции определим компакты $J_{m}^{\alpha}$, семейства $\mathscr{C}_{\alpha}^{m}, \alpha \geqslant 1$, их замкнутых подмножеств и точки $p_{\alpha}=p_{\alpha}^{m}$ следующим образом.

0) $J_{m}^{0}=\left\{p_{0}\right\}$.

1) $J_{m}^{1}=T(m), \mathscr{C}_{1}^{m}=\left\{J_{m}^{1}\right\}, p_{1}=a_{1}$.

2) $J_{m}^{\alpha+1}=J_{m}^{\alpha} \times T(m), \mathscr{C}_{\alpha+1}^{m}=\left\{C \times T(m): c \in \mathscr{C}_{\alpha}^{m}\right\}, p_{\alpha+1}=p_{\alpha} \times\left\{a_{1}\right\}$.

$3)$ Если $\alpha$ предельно, то для всякого $\beta<\alpha$ возьмем экземпляр $A_{\alpha}^{\beta}$ множества $T(m) \backslash\left\{a_{2}\right\}$, пересекающийся с $J_{m}^{\beta}$ по концевой точке $a_{1}=p_{\beta}$, и положим $J_{m}^{\alpha}=A\left(\bigoplus_{\beta<\alpha} J_{m}^{\beta} \cup A_{\alpha}^{\beta}\right)(A(X)$ - александровская компактификация локально компактного пространства $X)$. Далее, $\mathscr{C}_{\alpha}^{m}=\left\{i_{\alpha}^{\beta}(C)\right.$ : $\left.C \in \mathscr{C}_{\beta}^{m}, \beta<\alpha\right\}$, а $p_{\alpha}$ - компактифицирующая точка. Здесь для каждого $\beta<\alpha$ через $i_{\alpha}^{\beta}={ }^{m} i_{\alpha}^{\beta}$ обозначено естественное вложение $J_{m}^{\beta} \rightarrow J_{m}^{\alpha}$.

Компакты $J_{m}^{\alpha}$ называются $m$-компактами Хендерсона, а элементы семейств $\mathscr{C}_{\alpha}^{m}-$ камерами компактов $J_{m}^{\alpha}$. Камеры гомеоморфны компактам $T_{n}$ и канонически замкнуты в $J_{m}^{\alpha}$.

Компакты $J_{2}^{\alpha}$ совпадают с компактами Хендерсона $J^{\alpha}$ [60]. Определение компактов $J_{m}^{\alpha}$ можно распространить на все порядковые числа $\alpha$.

Теорема 6.9. Для иельх $k, m \geqslant 2 u \alpha<\omega_{1}$ имеем

$$
\operatorname{dim}_{w k} J_{m}^{\alpha}=\operatorname{dim}_{k} J_{m}^{\alpha}=\operatorname{Ind} J_{m}^{\alpha}=\operatorname{Ind}_{\infty} J_{m}^{\alpha}=\alpha .
$$

СлеДСтвиЕ 6.10. Пусть $d$ - один из размерностных инвариантов, указанных в теореме 6.9. Тогда

$$
d J_{m}^{\alpha}=d J_{m+1}^{\alpha}=\alpha .
$$

ОПредЕЛЕНИЕ 6.11. Непрерывное отображение $f: X \rightarrow J_{m}^{\alpha}$ называется $c y$ щественным, если оно $w$-существенно на прообразе $f^{-1}(C)$ всякой камеры $C \in \mathscr{C}_{\alpha}^{m}$

Теорема 6.12. Если $X$ допускает существенное отображение в $J_{m}^{\alpha}$, то $\operatorname{dim}_{w m} X \geqslant \alpha$.

Обратить эту теорему нельзя. 
Пример 6.13. Для каждого $m \geqslant 2$ существует компакт $X_{m}$ размерности $\operatorname{dim}_{w m} X_{m}=\omega_{0}+1$, который не допускает существенного отображения в $J_{m}^{\omega_{0}+1}$.

Компакт $X_{2}$ построен Борстом (см. [52; пример 5.2.4]).

Теорема 6.14. Если $\operatorname{dim}_{w m} X \geqslant \alpha+1$, то $X$ допускает существенное отображение в $J_{m}^{\alpha}$.

Для пространства $X$ положим

$\operatorname{Ess}_{m} X=\sup \left\{\alpha<\omega_{1}: X\right.$ допускает существенное отображение в $\left.J_{m}^{\alpha}\right\}$.

Из теорем 6.12 и 6.14 получаем

$$
\operatorname{Ess}_{m} X \leqslant \operatorname{dim}_{w m} X \leqslant \operatorname{Ess}_{m} X+1
$$

Теорема 6.15. Если $\alpha<\omega_{1}-$ предельное число, то

$$
\operatorname{dim}_{w m} X \geqslant \alpha \Longleftrightarrow \operatorname{Ess}_{m} X \geqslant \alpha
$$

ТеОрема 6.16. Пусть $X$-метризуемый компакт, $C$-канторово множество и $\alpha<\omega_{1}$. Тогда

$$
\operatorname{dim}_{w m} X \geqslant \alpha \Longleftrightarrow \operatorname{Ess}_{m}(X \times C) \geqslant \alpha
$$

\section{§ 7. Наследственно сильно бесконечномерные компакты}

Приведем некоторые элементарные свойства существенности и несущественности по отношению к подпространству (см. определение 4.55). Все рассматриваемые пространства метризуемы и сепарабельны.

ОПРЕДЕЛЕниЕ 7.1. Семейство $\mathscr{A}$ подмножеств пространства $X$ называется допустимым для $X$, если

1) $A \in \mathscr{A}, B \subset A \Rightarrow B \in \mathscr{A}$;

2) для всякого $A \in \mathscr{A}$ существует окрестность $O A \in \mathscr{A}$;

$3)$ для дизъюнктных открытых множеств $G_{i} \in \mathscr{A}, i \in \omega$, их объединение $\bigcup_{i} G_{i}$ принадлежит $\mathscr{A}$.

Всюду в этом параграфе $\mathscr{U} \subset \operatorname{cov}_{m}(X)$ - счетное множество. Через $\mathscr{A}_{\mathscr{U}}$ обозначим семейство всех подмножеств пространства $X$, на которых семейство $\mathscr{U}$ несущественно.

ПРеДЛОЖЕНИЕ 7.2. Для сепарабельного метрического пространства X семейство $\mathscr{A}_{\mathscr{U}}$ допустимо для $X$.

ДоказАтеЛЬСтво. Свойство 7.1.1) очевидно. Проверим 7.1.2). Пусть $\mathscr{U}=$ $\left\{u_{i}: i \in \omega\right\}$ и $A \in \mathscr{A}_{\mathscr{U}}$. Поскольку $\mathscr{U}$ несущественно на $A$, существуют такие дизъюнктные открытые семейства $v_{i}$, вписанные в $u_{i}$, что

$$
A \subset V \equiv \bigcup\left\{\bigcup v_{i}: i \in \omega\right\}
$$

Множество $V$ и будет искомой окрестностью $O A$. 
Теперь 7.1.3). Пусть $u_{i}=\left\{U_{1}^{i}, \ldots, U_{k_{i}}^{i}\right\}$, а $\left\{G_{j}: j \in \omega\right\} \subset \mathscr{A}_{\mathscr{U}}-$ дизъюнктное открытое семейство. Для каждого $j$ существует такое дизъюнктное открытое семейство $v_{i}^{j}=\left\{{ }^{j} V_{1}^{i}, \ldots,{ }^{j} V_{k_{i}}^{i}\right\}$, что

$$
{ }^{j} V_{l}^{i} \subset U_{l}^{i} \quad \text { и } \quad G_{j} \subset \bigcup_{i, l}{ }^{j} V_{l}^{i} .
$$

Полагая $V_{l}^{i}=\bigcup_{j}\left(G_{j} \cap{ }^{j} V_{l}^{i}\right)$ и $v_{i}=\left\{V_{1}^{i}, \ldots, V_{k_{i}}^{i}\right\}$, получаем дизъюнктные открытые семейства $v_{i}$, вписанные в $u_{i}$ и удовлетворяющие условию

$$
\bigcup_{j} G_{j}=\bigcup\left\{\bigcup v_{i}: i \in \omega\right\} .
$$

Предложение 7.2 доказано.

Последовательность множеств $Y_{1}, Y_{2}, \ldots \subset X$ назовем открыто-дискретной, если у множеств $Y_{i}$ существуют дизъюнктные окрестности $G_{i}$. Из предложения 7.2 вытекает следующее утверждение.

ПРЕДЛОЖЕНИЕ 7.3. Если И несущественно на каждом элементе открьто-дискретного семейства $Y_{1}, Y_{2}, \ldots \subset X$, то $\mathscr{U}$ несущественно на $\bigcup_{i} Y_{i}$.

Для $Y \subset X$ полагаем

$$
\begin{gathered}
H_{c}(\mathscr{U}, Y)=\bigcup\{C: C-\text { компонента множества } Y, \\
\text { на которой } \mathscr{U} \text { несущественно }\} ; \\
H_{q}(\mathscr{U}, Y)=\bigcup\{Q: Q-\text { квазикомпонента множества } Y, \\
\text { на которой } \mathscr{U} \text { несущественно }\} .
\end{gathered}
$$

ПРЕДЛОЖЕНИЕ 7.4. Если множество $Y$ замкнуто в $X$, то $\mathscr{U}$ несущественно на $H_{c}(\mathscr{U}, Y)$.

ДокАЗАТЕЛЬСтво. Пусть $C$ - компонента $Y$, на которой $\mathscr{U}$ несущественно. По предложению 7.2 можно найти такое открыто-замкнутое в $Y$ множество $V_{C}$, что $C \subset V_{C}$ и $\mathscr{U}$ несущественно на $V_{C}$. В покрытие $\left\{V_{C}: C \subset H_{c}(\mathscr{U}, Y)\right\}$ множества $H_{c}(\mathscr{U}, Y)$ можно вписать счетное открыто-замкнутое покрытие. Тогда $\mathscr{U}$ существенно на $H_{c}(\mathscr{U}, Y)$ согласно предложению 7.3.

Следующее утверждение очевидно.

ПРЕДЛОЖЕНИЕ 7.5. Если $\mathscr{U}_{i}$ несущественно на $Y_{i}$, то $\bigcup_{i} \mathscr{U}_{i}$ несущественно на $\bigcup_{i} Y_{i}$.

ПрЕДЛОЖЕНИЕ 7.6. Пусть множество $Y \subset X$ открыто в $[Y]$. Тогда $\mathscr{U}_{1} \cup \mathscr{U}_{2}$ несущественно на

$$
H_{q}\left(\mathscr{U}_{1}, Y\right) \cap H_{q}\left(\mathscr{U}_{2}, Y\right)
$$

ДокАЗАТЕЛьство. Пусть $f:[Y] \rightarrow[0,1]$ - непрерывная функция с $Y=$ $f^{-1}(0,1]$. Положим $K_{n}=f^{-1}[1 /(n+1) ; 1 / n], n=1,2, \ldots$. Имеют место включения

$$
H_{q}\left(\mathscr{U}_{i}, Y\right) \cap K_{n} \subset H_{c}\left(\mathscr{U}_{i}, K_{n}\right), \quad i=1,2 .
$$


В самом деле, пусть $x \in H_{q}\left(\mathscr{U}_{i}, Y\right) \cap K_{n}$. Тогда $x$ принадлежит некоторой квазикомпоненте $Q$ множества $Y$, на которой $\mathscr{U}_{i}$ существенно. Пусть теперь $C$ - компонента множества $K_{n}$, содержащая точку $x$. Поскольку $K_{n}$ замкнуто в $Y$, имеем $C \subset Q$. Итак, условие (7.1) проверено.

По предложению 7.4 семейство $\mathscr{U}_{i}$ несущественно на $H_{c}\left(\mathscr{U}_{i}, K_{n}\right)$. Значит, согласно предложению $7.3 \mathscr{U}_{1}$ несущественно на $Y_{1}=\bigcup\left\{H_{c}\left(\mathscr{U}_{1}, K_{2 n-1}\right): n \geqslant 1\right\}$, а $\mathscr{U}_{2}$ несущественно на $Y_{2}=\bigcup\left\{H_{c}\left(\mathscr{U}_{2}, K_{2 n}\right), n \geqslant 1\right\}$. По предложению 7.5 $\mathscr{U}_{1} \cup \mathscr{U}_{2}$ несущественно на $Y_{1} \cup Y_{2}$, а из (7.1) вытекает, что

$$
H_{q}\left(\mathscr{U}_{1}, Y\right) \cap H_{q}\left(\mathscr{U}_{2}, Y\right) \subset Y_{1} \cup Y_{2}
$$

Предложение 7.6 доказано.

Теорема 7.7. Пусть $X-$ компакт и $\mathscr{U} \subset \operatorname{cov}_{m}(X)$ - счетное семейство, существенное на $X$. Тогда существует такое открытое множество $G \subset X$, что $\mathscr{U}$ несущественно на $G$ и для всякого множества $Y \subset X \backslash G$ положительной размерности существует бесконечное семейство $\mathscr{V} \subset \mathscr{U}$, которое существенно на $Y$.

ДокАЗАТЕЛЬСтво. Представим $\mathscr{U}$ в виде дизъюнктной суммы бесконечных подсемейств:

$$
\mathscr{U}=\mathscr{U}_{1} \cup \mathscr{V}_{1} \cup \mathscr{U}_{2} \cup \mathscr{V}_{2} \cup \cdots
$$

Пусть $\mathscr{F}$ - счетное семейство непрерывных функций $X \rightarrow[0,1]$, разделяющее точки $X$, а $\mathscr{K}$ - такое счетное семейство канторовых подмножеств отрезка $[0,1]$, что каждый невырожденный интервал отрезка $[0,1]$ содержит некоторое $K \in \mathscr{K}$. Запишем произведение $\mathscr{F} \times \mathscr{K}$ в виде последовательности $\left(f_{1}, K_{1}\right)$, $\left(f_{2}, K_{2}\right), \ldots$.

Пусть $g_{i}: K_{i} \rightarrow \exp X-$ непрерывная сюръекция. Положим $Y_{i}=\bigcup\left\{f_{i}^{-1}(t) \backslash\right.$ $\left.g_{i}(t): t \in K_{i}\right\}=f_{i}^{-1}\left(K_{i}\right) \backslash \bigcup\left\{f_{i}^{-1}(t) \cap g_{i}(t): t \in K_{i}\right\}$. Множество $\bigcup\left\{f_{i}^{-1}(t) \cap g_{i}(t)\right.$ : $\left.t \in K_{i}\right\}$ замкнуто в $X$ и, следовательно, $Y_{i}$ открыто в [ $\left.Y_{i}\right]$. По предложению 7.6 семейство $\mathscr{U}_{i} \cup \mathscr{V}_{i}$ несущественно на $Z_{i}=H_{q}\left(\mathscr{U}_{i}, Y_{i}\right) \cap H_{q}\left(\mathscr{V}_{i}, Y_{i}\right)$. Значит, $\mathscr{U}$ несущественно на $Z=Z_{1} \cup Z_{2} \cup \cdots$. По предложению 7.2 существует окрестность $G$ множества $Z$, на которой $\mathscr{U}$ несущественно. Покажем, что $G$ - искомое множество.

Пусть $Y \cap G=\varnothing$ и $\operatorname{dim} Y>0$. Отображение $x \rightarrow(f(x))_{f \in \mathscr{F}}$ вкладывает $X$ в $\prod_{f \in \mathscr{F}} f(X)$. Значит, $Y$ вложено в $\prod_{f \in \mathscr{F}} f(Y)$. Поэтому $\operatorname{dim} f(Y)>0$ для некоторого $f \in \mathscr{F}$. Следовательно, $f(Y)$ содержит невырожденный интервал и вместе с ним некоторое канторово множество $K \in \mathscr{K}$. Возьмем $i$, для которого $\left(f_{i}, K_{i}\right)=(f, K)$, и покажем, что либо $\mathscr{U}_{i}$, либо $\mathscr{V}_{i}$ существенно на $Y$.

Предположим, что это не так. Тогда по предложению 7.2 существует окрестность $V \supset Y$, на которой оба семейства $\mathscr{U}_{i}$ и $\mathscr{V}_{i}$ несущественны. Из существенности $\mathscr{U}$ на $X$ вытекает, что $X \backslash V \neq \varnothing$. Следовательно, существует такое $t \in K_{i}$, что $g_{i}(t)=X \backslash V$. Положим $D_{i}=f_{i}^{-1}(t) \backslash g_{i}(t)$. Очевидно, что $D_{i}=\bigcap\left\{f_{i}^{-1}(U) \cap Y_{i}: U\right.$ содержит $t$ и открыто-замкнуто в $\left.K_{i}\right\}$.

Множества $f_{i}^{-1}(U) \cap Y_{i}$ открыто-замкнуты в $Y_{i}$. Следовательно, всякая квазикомпонента множества $Y_{i}$, пересекающаяся с $D_{i}$, содержится в $D_{i}$. Семейства $\mathscr{U}_{i}$ и $\mathscr{V}_{i}$ несущественны на $D_{i}$, так как $D_{i} \subset V$. Значит, $D_{i} \subset H_{q}\left(\mathscr{U}_{i}, Y_{i}\right) \cap$ 
$H_{q}\left(\mathscr{V}_{i}, Y_{i}\right)$, т.е. $D_{i} \subset Z_{i}$. Поскольку $f_{i}(Y) \supset K_{i}$, множество $D_{i}$ пересекается с $Y$. Тогда, тем более, $Z_{i} \cap Y \neq \varnothing$. Но это противоречит тому, что $Y \cap G=\varnothing$.

Теорема 7.8. Предположим, что компакт $X$ не является $m$-C-пространством. Тогда $X$ содержит такой компакт $Z \notin m-C$, что никакое множество $Y \subset Z$ положительной размерности не является $m$-C-пространством.

ДоказАтельство. Поскольку $X \notin m$ - $C$, существует множество $\mathscr{U}_{0}=\left\{u_{0}\right.$, $\left.u_{1}, \ldots\right\} \subset \operatorname{cov}_{m}(X)$, существенное на $X$. Положим $\mathscr{U}=\mathscr{U}_{0} \backslash\left\{u_{0}\right\}$. Семейство $\mathscr{U}$ также существенно на $X$. Возьмем открытое множество $G \subset X$, удовлетворяющее теореме 7.7 , и положим $Z=X \backslash G$. Нам надо показать только, что $\operatorname{dim} Z>0$. Предположим, что $\operatorname{dim} Z \leqslant 0$. Пусть $u_{0}=\left\{U_{1}^{0}, \ldots, U_{k}^{0}\right\}$. Существует дизъюнктное открытое семейство $v_{0}=\left\{V_{1}^{0}, \ldots, V_{k}^{0}\right\}$, вписанное в $u_{0}$ и покрывающее $Z$. Положим $F=X \backslash V_{1}^{0} \cup \cdots \cup V_{k}^{0}$. Поскольку $F \subset G$ и $\mathscr{U}$ несущественно на $G$, существуют такие дизъюнктные открытые семейства $v_{i}$, вписанные в $u_{i}$, $i=1,2, \ldots$, что $F \subset \bigcup\left\{\bigcup v_{i}: i \geqslant 1\right\}$. Тогда семейство $\bigcup\left\{v_{i}: i \geqslant 0\right\}$ является покрытием $X$, что противоречит существенности $\mathscr{U}$ на $X$. Теорема 7.8 доказана.

Теорема 7.7 для $m=2$ и $m=\infty$ доказана М. Левиным [62]. Теорему 7.8 для $m=2$ получил Л. Рубин [63] после того, как Дж. Уолш [64] построил первый пример наследственно бесконечномерного пространства. Для $m=\infty$ и замкнутых $Y$ теорему 7.8 доказал Р. Поль [65]. Первым же, кто доказал теорему типа 7.8, был А. В. Зарелуа: $m=2$, а $Y$ замкнуто [66].

Так же, как и теорема 7.8, доказывается следующее утверждение.

ТеОрема 7.9. Предположим, что компакт $X$ не является $w$-m-C-nространством. Тогда $X$ содержит такой компакт $Z \notin w-m-C$, что никакое множество $Y \subset Z$ положительной размерности не является $w-m$-C-пространством.

ЗАмЕчАНИЕ 7.10. Если сильно бесконечномерные пространства назвать 2-сильно бесконечномерными, то по аналогии возникают $m(w-m)$-сильно бесконечномерные пространства. Поэтому теоремы 7.8 и 7.9 можно сформулировать так:

во всяком $m(w-m)$-силъно бесконечномерном компакте содержится наследственно $m(w-m)$-сильно бесконечномерный компакт.

\section{Добавление. Промежуточные размерности бикомпактов}

Всякий метризуемый бикомпакт $X$ конечной размерности $\operatorname{dim} X>0$ содержит одномерные континуумы. То же самое относится и к бикомпактам, трансфинитные размерности ind и Ind которых определены. Из результатов $\S 7$ вытекает, что всякий метризуемый компакт $X$, не являющийся $C$-пространством, содержит бесконечномерный компакт $Z$, не имеющий подпространств конечной положительной размерности. В частности, слабо бесконечномерный компакт Борста $E_{\omega_{0}}$ из [8] содержит компакт $Z_{0}$, не имеющий подпространств конечной положительной размерности. Будучи слабо бесконечномерным, компакт $Z_{0}$ имеет трансфинитную размерность $\operatorname{dim}_{2} Z_{0}=\alpha<\omega_{1}$, но не содержит компактов $Y$ размерности $\operatorname{dim}_{2} Y=\operatorname{dim} Y=1$. 
Вопрос А.1. Существует ли бесконечномерный метризуемый $C$-компакт $X$, не содержащий подкомпактов $Y$ размерности $0<\operatorname{dim}_{\infty} Y<\operatorname{dim}_{\infty} X$ ?

Более слабой версией этого вопроса является

Вопрос А.2. Существует ли бесконечномерный метризуемый $C$-компакт $X$, не содержащий одномерных континуумов?

Возникает следующее определение.

ОПРЕДЕЛЕНИЕ А.3. Пусть $d$ - размерностный инвариант, принимающий конечные или трансфинитные значения. Для пространства $X$ полагаем

$$
d \text {-sc }(X)=\{\alpha: \exists \text { замкнутое } F \subset X \text { размерности } d(F)=\alpha\} \text {. }
$$

Множество $d$-sc $(X)$ называется $d$-шкалой пространства $X$. Если

$$
d-\operatorname{sc}(X)=\{\alpha: \alpha \leqslant d(X)\}
$$

то $d$-шкала $d$-sc $(X)$ называется элементарной. $d$-шкала $d$-sc $(X)$ называется сложной, если она не элементарна и $1 \in d$-sc $(F)$ для всякого замкнутого множества $F \subset X$ размерности $d(F) \geqslant 1$. Условившись, что $\operatorname{dim} X=\omega_{0}$, если $\operatorname{dim} X=\infty$, отнесем к числу функций $d$ и лебегову размерность. Шкалой пространства $X$ называется его $\operatorname{dim}-ш к а л а ~ \operatorname{dim}-\mathrm{sc}(X) \equiv \operatorname{sc}(X)$.

Компакт Хендерсона [67] является первым примером метризуемого компакта с неэлементарной шкалой. Бикомпакт без промежуточных размерностей из [68] - первый пример конечномерного бикомпакта с неэлементарной шкалой.

Вопрос А.4. Существует ли метризуемый компакт со сложной шкалой?

ЗАмечАние А.5. Из теоремы $7.8(m=\infty)$ вытекает, что метризуемый компакт со сложной шкалой обязан быть $C$-пространством. Теорема А.8 показывает, что для неметризуемых бикомпактов это не так.

ОПредЕЛЕНИЕ А.6. Шкала $\operatorname{sc}(X)$ называется устойчивой, если $\operatorname{sc}(X)=$ $\operatorname{sc}(F)$ для всякого замкнутого множества $F \subset X$ размерности $\operatorname{dim} F=\operatorname{dim} X$. Шкала $\operatorname{sc}(X)$ называется монотонно устойчивой, если

$$
\operatorname{sc}(F)=[-1 ; \operatorname{dim}(F)] \cap \operatorname{sc}(X)
$$

для всякого замкнутого множества $F \subset X$.

Теорема А.7. Для всякого множества $s \subset\left[1 ; \omega_{0}\right]$, содержащего -1 u 0 , существует $C$-бикомпакт $X(s)$ с первой аксиомой счетности, для которого $s$ является монотонно устойчивой шкалой.

Теорема A.8. Для всякого множества $s \subset\left[1, \omega_{0}\right]$, содержащего $-1,0 u$ $\omega_{0}$, существует сильно бесконечномерный бикомпакт $Y(s)$ с первой аксиомой счетности, для которого $s$ является монотонно устойчивой шкалой и всякое несчетномерное замкнутое подмножество которого сильно бесконечномерно. 
Tеорема A.9 (CH). Для всякого множества $s \subset\left[-1, \omega_{0}\right]$, содержащего -1 и 0, существует совершенно нормалъный $C$-бикомпакт $X_{0}(s)$, для которого $s$ явлется монотонно устойчивой шкалой.

Tеорема A.10 (CH). Для всякого множества $s \subset\left[-1, \omega_{0}\right]$, содержащего $-1,0$ и $\omega_{0}$, существует совершенно нормальный сильно бесконечномерный бикомпакт $Y_{0}(s)$, для которого $s$ является монотонно устойчивой шкалой и всякое несчетномерное замкнутое подмножество которого сильно бесконечномерно.

ЗАКЛЮчИТЕЛЬНОЕ ЗАМЕЧАНИЕ. Как отмечено во введении, статья не является полным обзором теории слабо бесконечномерных пространств. Однако мы приводим список дополнительной литературы [69]-[102], который позволит читателю более обстоятельно ознакомиться с рассматриваемой тематикой.

ПРИМЕЧАНИЕ ПРИ КОРРЕКТУРЕ. С применением теоремы 6.4 автор недавно доказал, что класс $S-w-\infty-C$ совпадает с классом $S$-wid.

\section{Список литературы}

[1] П.С. Александров, "О гомологических свойствах расположения комплексов и замкнутых множеств", Изв. АН СССР. Сер. матем., 6 (1942), 227-282.

[2] А.Н. Дранишников, "Гомологическая теория размерности", УМН, 43:4 (1988), 11-55; англ. пер.: А. N. Dranishnikov, "Homological dimension theory", Russian Math. Surveys, 43:4 (1988), 11-63.

[3] П.С. Александров, "Предисловие к русскому переводу", в кн.: В. Гуревич, Г. Волмэн, Теория размерности, ИЛ, М., 1948.

[4] Б. Т. Левшенко, "О сильно бесконечномерных пространствах", Вестн. Моск. унта. Сер. матем., мех., астрон., физ., хим., 1959, № 5, 219-228.

[5] Е. Г. Скляренко, "О размерностных свойствах бесконечномерных пространств", Изв. АН СССР. Сер. матем., 23 (1959), 197-212.

[6] D. W. Henderson, "Each strongly infinite-dimensional compactum contains a hereditarily infinite-dimensional compact subset", Amer. J. Math., 89:1 (1967), 122-123.

[7] R. Pol, "A weakly infinite-dimensional compactum which is not countable-dimensional", Proc. Amer. Math. Soc., 82:4 (1981), 634-636.

[8] P. Borst, A weakly infinite-dimensional compactum not having Property $C$, Preprint, Vrije Universiteit, Amsterdam, 2005; Fund. Math. (to appear).

[9] W. E. Haver, "A covering property for metric spaces", Topology conference (Blacksburg, VA, 1973), Lecture Notes in Math., 375, Springer, Berlin, 1974, 108-113.

[10] D. F. Addis, J.H. Gresham, "A class of infinite-dimensional spaces. I. Dimension theory and Alexandroff's problem", Fund. Math., 101:3 (1978), 195-205.

[11] F.D. Ancel, "Proper hereditary shape equivalences preserve property C", Topology Appl., 19:1 (1985), 71-74.

[12] V. Gutev, V. Valov, "Continuons selections and C-spaces", Proc. Amer. Math. Soc., 130:1 (2002), 233-242.

[13] V. V. Uspenskij, "A selection theorem for C-spaces", Topology Appl., 85:1-3 (1998), 351-374.

[14] V. Valov, "Continuous selections and finite $C$-spaces", Set-Valued Anal., 10:1 (2002), $37-51$.

[15] В. В. Федорчук, "О некоторых классах слабо бесконечномерных пространств", Фундам. и прикл. матем. (в печати). 
[16] V. V. Fedorchuk, "Questions on weakly infinite-dimensional spaces", Open problems in topology II, ed. E. M. Pearl, Elsevier, Amsterdam, 2007 (to appear).

[17] П. С. Александров, Б. А. Пасынков, Введение в теорию размерности. Введение в теорию топологических пространств и общую теорию размерности, Наука, M., 1973.

[18] R. Engelking, Theory of dimensions, finite and infinite, Sigma Ser. Pure Math., 10, Heldermann, Lemgo, 1995.

[19] R. Pol, H. Toruńczyk, "Topics in dimension theory", Recent progress in general topology, II (Prague, Czech Republic, 2001), eds. M. Hušek, J. van Mill, Elsevier, Amsterdam, 2002, 395-421.

[20] P. Urysohn, "Mémoire sur les multiplicités Cantoriennes", Fund. Math., 7 (1925), 30-137.

[21] W. Hurewicz, "Über unendlich-dimensionale Punktmengen", Proc. Akad. Amsterdam, 31 (1928), 916-922.

[22] Ю. М. Смирнов, "Об универсальных пространствах для некоторых классов бесконечномерных пространств", Изв. АН СССР. Сер. матем., 23 (1959), 185-196.

[23] В. В. Федорчук, "О некоторых вопросах топологической теории размерности", УМH, 57:2 (2002), 139-178; англ. пер.: V. V. Fedorchuk, "On some problems of topological dimension theory", Russian Math. Surveys, 57:2 (2002), 361-398.

[24] Б. А. Пасынков, В.В. Федорчук, В.В.Филиппов, “Теория размерности", Итоги науки и техники. Алгебра. Топология. Геометрия, 17, ВИНИТИ, М., 1979, 229-306; англ. пер.: В. A. Pasynkov, V. V. Fedorchuk, V. V. Filippov, "Dimension theory", J. Sov. Math., 18 (1982), 789-841.

[25] P. Urysohn, "Mémoire sur les multiplicités Cantoriennes (suite)", Fund. Math., 8 (1926), 225-359.

[26] W. Hurewicz, H. Wallman, Dimension theory, Princeton Math. Ser., 4, Princeton Univ. Press, Princeton, NJ, 1941.

[27] W. E. Haver, "Locally contractible spaces that are absolute neighborhood retracts", Proc. Amer. Math. Soc., 40:1 (1973), 280-284.

[28] R. H. Bing, "Metrisation of topological spaces", Canad. J. Math., 3 (1951), 175-186.

[29] K. Nagami, "Paracompactness and strong screenability", Nagoya Math. J., 8 (1955), 83-88.

[30] P. A. Ostrand, "Covering dimension in general spaces", General Topology and Appl., 1:3 (1971), 209-221.

[31] В. А. Чатырко, "Слабо бесконечномерные пространства”, УМН, $46: 3$ (1991), 161177; англ. пер.: V.A. Chatyrko, "Weakly infinite-dimensional spaces", Russian Math. Surveys, 46:3 (1991), 191-210.

[32] М. Катетов, “О продолжении локально конечных покрытий”, Colloq. Math., 6 (1958), 145-151.

[33] C. H. Dowker, "Homotopy extension theorems", Proc. London Math. Soc. (3), 6:1 (1956), 100-116.

[34] E. Michael, "Point-finite and locally finite coverings", Canad. J. Math., 7 (1955), $275-279$.

[35] В. В. Федорчук, "Бесконечномерные бикомпакты", Изв. АН СССР. Сер. матем., 42:5 (1978), 1162-1178; англ. пер.: V. V. Fedorčuk, "Infinite-dimensional compact Hausdorff spaces", Math. USSR-Izv., 13:2 (1979), 445-460.

[36] L. A. Steen, "A direct proof that a linearly ordered space is hereditarily collectionwise normal", Proc. Amer. Math. Soc., 24:4 (1970), 727-728.

[37] Н. Хаджииванов, "О бесконечномерных пространствах", Bull. Acad. Polon. Sci. Sér. Sci. Math. Astronom. Phys., 19 (1971), 491-500.

[38] L. Polkowski, "Some theorems on invariance of infinite dimension under open and closed mappings", Fund. Math., 119:1 (1983), 11-34. 
[39] K. Morita, "On the dimension of normal spaces. II", J. Math. Soc. Japan, 2 (1950), $16-33$.

[40] Б. Т. Левшенко, "О бесконечномерных пространствах", Докл. АН СССР, 139:2 (1961), 286-289; англ. пер.: В. Т. Levšenko, "On infinite dimensional spaces", Soviet Math. Dokl., 2, 915-918.

[41] А.В. Зарелуа, "О теореме Гуревича", Докл. АН СССР, 14:4 (1961), 777-780; англ. пер.: A. V. Zarelua, "On a theorem of Hurewicz", Soviet Math. Dokl., 2 (1961), $1534-1537$.

[42] Ю. М. Смирнов, “О трансфинитной размерности”, Матем. сб., 58(100):4 (1962), 415-422.

[43] А.И. Вайнштейн, "Об одном классе бесконечномерных пространств", Матем. сб., 79(121):3(7) (1969), 433-443; англ. пер.: A. I. VaŭnšteĬn, "On a class of infinitedimensional spaces", Math. USSR-Sb., 8:3 (1969), 409-418.

[44] Y. Hattori, K. Yamada, "Closed pre-images of C-spaces", Math. Japon., 34:4 (1989), $555-561$.

[45] В. В. Федорчук, "Вполне замкнутые отображения и их приложения", Фундам. и прикл. матем., 9:4 (2003), 105-235; англ. пер.: V. V. Fedorchuk, "Fully closed mappings and their applications", J. Math. Sci. (N. Y.), 136:5 (2006), 4201-4292.

[46] Е. Г. Скляренко, "Две теоремы о бесконечномерных пространствах", Докл. АН CCCP, 143 (1962), 1053-1056; англ. пер.: E. G. Sklyarenko, "Two theorems on infinite-dimensional spaces", Soviet Math. Dokl., 3 (1962), 547-550.

[47] R. Pol, "On classification of weakly infinite-dimensional compacta", Fund. Math., 116:3 (1983), 169-188.

[48] P. Borst, "Classification of weakly infinite-dimensional spaces. I. A transfinite extension of the covering dimension", Fund. Math., 130:1 (1988), 1-25.

[49] В.А. Чатырко, "Классификация компактов со свойством С", Сиб. матем. журн., 33:6 (1992), 216-220; англ. пер.: V.A. Chatyrko, "Classification of compacta with the property C", Siberian Math. J., 33:6 (1992), 1144-1148.

[50] K. Kuratowski, Topology, vol. I, Academic Press, New York-London; Państwowe Wydawnictwo Naukowe, Warsaw, 1966; рус. пер.: К. Куратовский, Топология, т. I, Мир, М., 1966.

[51] P. Borst, "Some remarks concerning C-spaces", Topology Appl., 154:3 (2007), 665674.

[52] P. Borst, "Classfication of weakly infinite-dimensional spaces II. Essential mappings", Fund. Math., 130:2 (1988), 73-99.

[53] K. Yokoi, "Compactification and factorization theorems for transfinite covering dimension", Tsukuba J. Math., 15:2 (1991), 389-395.

[54] V. A. Chatyrko, "On the transfinite dimension dim", Questions Answers Gen. Topology, 9:2 (1991), 177-194.

[55] V.A. Chatyrko, "Classification of metric compacta with property C", Questions Answers Gen. Topology, 10:1 (1992), 51-62.

[56] V.A. Chatyrko, "On factorization theorem for transfinite dimension $\operatorname{dim}_{C}$ ", Sci. Math., 3:3 (2000), 357-366.

[57] R. Engelking, R. Pol, "Compactificftions of countable-dimensional and strongly countable-dimensional spaces", Proc. Amer. Math. Soc., 104:3 (1988), 985-987.

[58] Б. А. Пасынков, "О размерности нормальных пространств", Докл. АН СССР, 201:5 (1971), 1049-1052; англ. пер.: В. A. Pasynkov, "On the dimension of normal spaces", Soviet Math. Dokl., 12 (1971), 1784-1787.

[59] C. H. Dowker, "Local dimension of normal spaces", Q. J. Math., 6:1 (1955), 101-120.

[60] D. W. Henderson, "A lower bound for transfinite dimension", Fund. Math., 63 (1968), 167-173. 
[61] P. Borst, J.J. Dijkstra, "Essential mappings and transfinite dimension", Fund. Math., 125:1 (1985), 41-45.

[62] M. Levin, "Inessentiality with respect to subspaces", Fund. Math., 147:1 (1995), 93-98.

[63] L. R. Rubin, "Hereditarily strongly infinite dimensional spaces", Michigan Math. J., 27:1 (1980), 65-73.

[64] J. J. Walsh, "Infinite dimensional compacta containing no $n$-dimensional $(n \geqslant 1)$ subsets", Topology, 18:1 (1979), 91-95.

[65] R. Pol, "On light mappings without perfect fibers on compacta", Tsukuba J. Math., 20:1 (1996), 11-19.

[66] А. В. Зарелуа, "Построение сильно бесконечномерных компактов с помощью колец непрерывных функций”, Докл. АН ССCP, 214 (1974), 264-267; англ. пер.: A. V. Zarelua, "Construction of strongly infinite-dimensional compacta using rings of continuous functions", Soviet Math. Dokl., 15 (1974), 106-110.

[67] D. W. Henderson, "Finite dimensional subsets of infinite dimensional spaces", Topology Seminar (Wisconsin, 1965), Ann. of Math. Stud., 60, Princeton Univ. Press, Princeton, NJ, 1966, 141-146.

[68] В.В. Федорчук, "Бикомпакты без промежуточных размерностей", Докл. АН CCCP, 213 (1973), 795-797; англ. пер.: V. V. Fedorchuk, "Bicompact spaces without intermediate dimensions", Soviet Math. Dokl., 14 (1973), 1808-1811.

[69] F. D. Ancel, "The role of countable dimensionality in the theory of cell-like relations", Trans. Amer. Math. Soc., 287:1 (1985), 1-40.

[70] P. Borst, "Spaces having a weakly-infinite-dimensional compactification", Topology Appl., 21:3 (1985), 261-268.

[71] P. Borst, "On weakly infinite-dimensional subspaces", Fund. Math., 140:3 (1992), $225-235$.

[72] V. A. Chatyrko, "On the transfinite dimension dim and essential mappings", Topology Proc., 20 (1995), 67-73.

[73] A. Chigogidze, V. Valov, "The extension dimension and $C$-spaces", Bull. London Math. Soc., 34:6 (2002), 708-716.

[74] М. М. Чобан, "Отображения и размерностные свойства пространств", Топология, Тр. МИАН, 154, Наука, М., 1983, 296-305; англ. пер.: M. M. Choban, "Mappings and dimension properties of spaces", Proc. Steklov Inst. Math., 154 (1984), 317-326.

[75] A. N. Dranishnikov, "Stable cohomotopy dimension and weakly infinite dimensional spaces", Topology Appl., 47:1 (1992), 79-81.

[76] R. Engelking, General topology, Sigma Ser. Pure Math., 6, Heldermann, Berlin, 1989.

[77] В. В. Федорчук, “Формула Гуревича для слабо бесконечномерных вполне замкнутых отображений”, Вестн. Моск. ун-та. Сер. 1. Матем., мех., 2006, №4, $54-55$.

[78] D. J. Garity, "Property $C$ and closed maps", Topology Appl., 26:2 (1987), 125-130.

[79] D. J. Garity, D. M. Rohm, "Property C, refinable maps and dimension raising maps", Proc. Amer. Math. Soc., 98:2 (1986), 336-340.

[80] J. H. Gresham, "A class of infinite-demensional spaces. II. An extension theorem and the theory of retracts", Fund. Math., 107:3 (1980), 237-245.

[81] N. G. Hadziivanov, "A lower bound for the cardinal dimension of certain $G_{\delta}$ sets in Tychonoff cube", C. R. Acad. Bulgare Sci., 28 (1975), 151-152.

[82] M. Levin, "A short construction of hereditarily infinite dimensional compacta", Topology Appl., 65:1 (1995), 97-99.

[83] M. Levin, J. T. Rogers, "A generalization of Kelley's theorem for $C$-spaces", Proc. Amer. Math. Soc., 128:5 (2000), 1537-1541. 
[84] А. Лелек, "О размерности наростов при компактных расширениях", Докл. АН CCCP, 160 (1965), 534-537; англ. пер.: A. Lelek, "On the dimension of remainders in compact extensions", Soviet Math. Dokl., 6 (1965), 136-140.

[85] И. М. Лейбо, "О замкнутых отображениях бесконечно-мерных пространств”, Докл. АН ССCP, 199 (1971), 533-535; англ. пер.: I. M. Leibo, "On closed mappings of infinite-dimensional spaces", Soviet Math. Dokl., 12 (1971), 1111-1113.

[86] M. Levin, L. R. Rubin, P. J. Schapiro, "The Mardešić factorization theorem for extension theory and C-separation", Proc. Amer. Math. Soc., 128:10 (2000), 3099-3106.

[87] E. Pol, "A weakly infinite-dimensional space whose product with the irrationals is strongly infinite-dimensional", Proc. Amer. Math. Soc., 98:2 (1986), 349-352.

[88] E. Pol, "Spaces whose $n$th power is weakly intinite-dimensional but whose $(n+1)$ th power is not", Proc. Amer. Math. Soc., 117:3 (1993), 871-876.

[89] R. Pol, "A remark on A-weakly infinite-dimensional spaces", Topology Appl., 13:1 (1982), 97-101.

[90] R. Pol, "Countable dimensional universal sets", Trans. Amer. Math. Soc., 297:1 (1986), 255-268.

[91] R. Pol, "Selected topics related to countable-dimensional metrizable spaces", General topology and its relations to modern analysis and algebra, VI (Prague, 1986), Res. Exp. Math., 16, Heldermann, Berlin, 1988, 421-436.

[92] L. Polkowski, "A sum theorem for A-weakly infinite-dimensional spaces", Fund. Math., 119:1 (1983), 7-10.

[93] T. Radul, "Absorbing spaces for C-compacta", Topology Appl., 83:2 (1998), 127-133.

[94] D. M. Rohm, "Products of infinite-dimensional spaces", Proc. Amer. Math. Soc., 108:4 (1990), 1019-1023.

[95] L. R. Rubin, "Noncompact hereditarily strongly infinite dimensional spaces", Proc. Amer. Math. Soc., 79:1 (1980), 153-154.

[96] L. R. Rubin, R. M. Schori, J. J. Walsh, "New dimension-theory techniques for constructing infinite-dimensional examples", General Topology Appl., 10:1 (1979), 93102.

[97] Е. В. Щепин, “Функторы и несчетные степени компактов”, УМН, 36:3 (1981), 3-62; англ. пер.: Е. V. Shchepin, "Functors and uncountable powers of compacta", Russian Math. Surveys, 36:3 (1981), 1-71.

[98] J. Segal, T. Watanabe, "Universal maps snd infinite-dimensional spaces", Bull. Polish Acad. Sci. Math., 39:3-4 (1991), 225-228.

[99] Е. Г. Скляренко, "Несколько замечаний о бесконечномерных пространствах", Докл. АН СССР, 126 (1959), 1203-1206.

[100] V.V. Uspenskij, "A note on question of R. Pol concerning light maps", Topology Appl., 103:3 (2000), 291-294.

[101] K. Yamada, "Mapping and product theorems for infinite-dimensional spaces", Glas. Mat. Ser. III, 23(43):1 (1988), 193-202.

[102] А.В. Зарелуа, "О наследственно бесконечномерных пространствах", Theory of sets and topology, in honour of Felix Hausdorff, 1868-1942, VEB Deutsch. Verlag Wissensch., Berlin, 1972, 509-525.

В. В. Федорчук (V. V. Fedorchuk)

Московский государственный университет

Поступила в редакцию

им. М. В. Ломоносова

E-mail: fedorch@tsi.ru

25.08.2006 